\title{
A contrail cirrus prediction model
}

\author{
U. Schumann \\ Deutsches Zentrum für Luft- und Raumfahrt, Institut für Physik der Atmosphäre, Oberpfaffenhofen, Germany \\ Correspondence to: U. Schumann (ulrich.schumann@dlr.de)
}

Received: 27 October 2011 - Published in Geosci. Model Dev. Discuss.: 28 November 2011

Revised: 6 April 2012 - Accepted: 10 April 2012 - Published: 3 May 2012

\begin{abstract}
A new model to simulate and predict the properties of a large ensemble of contrails as a function of given air traffic and meteorology is described. The model is designed for approximate prediction of contrail cirrus cover and analysis of contrail climate impact, e.g. within aviation system optimization processes. The model simulates the full contrail life-cycle. Contrail segments form between waypoints of individual aircraft tracks in sufficiently cold and humid air masses. The initial contrail properties depend on the aircraft. The advection and evolution of the contrails is followed with a Lagrangian Gaussian plume model. Mixing and bulk cloud processes are treated quasi analytically or with an effective numerical scheme. Contrails disappear when the bulk ice content is sublimating or precipitating. The model has been implemented in a "Contrail Cirrus Prediction Tool" (CoCiP). This paper describes the model assumptions, the equations for individual contrails, and the analysis-method for contrailcirrus cover derived from the optical depth of the ensemble of contrails and background cirrus. The model has been applied for a case study and compared to the results of other models and in-situ contrail measurements. The simple model reproduces a considerable part of observed contrail properties. Mid-aged contrails provide the largest contributions to the product of optical depth and contrail width, important for climate impact.
\end{abstract}

\section{Introduction}

Contrails are thin linear ice particle clouds which form in the atmosphere behind cruising aircraft because of mixing of the emitted water vapor with cold ambient air leading to local liquid saturation, condensation of water on aerosols, and subsequent freezing (Schmidt, 1941; Appleman, 1953; Schumann, 1996). In ice-supersatured air masses contrails spread and grow by uptake of ambient water. The total ice mass content may be several orders of magnitude larger than the amount of water emitted from the aircraft (Knollenberg, 1972; Heymsfield et al., 1998; Atlas et al., 2006). Contrails have often been observed to persist for many hours and transform into contrail cirrus, with considerable cloud cover and optical depth (Detwiler and Pratt, 1984; Schumann and Wendling, 1990; Bakan et al., 1994; Minnis et al., 1998; Duda et al., 2001; Mannstein and Schumann, 2005; Atlas et al., 2006; Vazquez-Navarro, 2009; Atlas and Wang, 2010). Persisting contrails are often associated with, or embedded in thin cirrus (Sassen, 1997; Immler et al., 2008). In regions with high traffic density, high humidity, and thin cirrus, contrails may spread quickly over a large region. Such so-called contrail outbreaks may cause a considerable fraction of the annual mean contrail cover (Duda et al., 2001; Haywood et al., 2009). Presently, more than 80000 commercial flights per day are performed globally and traffic is increasing (Wilkerson et al., 2010). A large fraction of the aircraft cruises at altitudes where contrails may form (Appleman, 1953; Miake-Lye et al., 1993; Sausen et al., 1998). Contrails may provide surfaces for heterogeneous chemistry (Meilinger et al., 2005; Voigt et al., 2010). Contrails are visible tracers of aircraft impact on the atmosphere. In spite of still large uncertainties, the climate impact of contrails appears to be important (Fahey et al., 1999; IPCC, 2007; Lee et al., 2010; Burkhardt and Kärcher, 2011).

In order to assess the climate impact of individual aircraft flights, e.g. as input for route optimization (Mannstein et al., 2005), for aircraft optimization (Green, 2002; Koch et al., 2009; Schwartz and Kroo, 2011), or for prediction of contrail cover in an area with intense air traffic day by day (Duda et al., 2009), one needs a model which is able to compute contrail properties for individual flights as well as for a large fleet of aircraft regionally and globally with short computation times. 
Contrail and cirrus formation is a nonlinear process depending strongly on ambient meteorology and plume processes (Scorer and Davenport, 1970; Lee et al., 2010). These plume processes are inherently subgrid-scale in multidimensional global models (Schumann and Konopka, 1994; Cariolle et al., 2009; Burkhardt et al., 2010; Paoli et al., 2011). Plume ice cloud models with different degree of complexity for mixing and particle microphysics have been developed (Miake-Lye et al., 1993; Kärcher et al., 1996, 2009a; Brown et al., 1997; Jensen et al., 1998b; Kärcher, 1998; Meilinger et al., 2005; Naiman et al., 2010), but none of them treats the whole contrail life-cycle from contrail formation until dissipation.

The decision of whether a contrail forms or not along a given flight is a relatively simple task because it can be explained thermodynamically once the meteorological and aircraft parameters are sufficiently known (Schumann, 1996; Rädel and Shine, 2007). Weather and flight track data have been used to identify contrail forming flight tracks and follow their advection (Duda et al., 2004; Atlas et al., 2006; Duda et al., 2009). From comparisons with satellite data at computed contrail-positions, optical and sedimentation properties were successfully deduced (Duda et al., 2004). Contrail persistence analysis was compared with satellite-derived contrail cover showing high sensitivity to ice supersaturation and vertical wind input (Duda et al., 2009).

However, the formation of ice particles in the exhaust jet at time scales of $0.1 \mathrm{~s}$ to $20 \mathrm{~s}$ (Kärcher et al., 1998; Paoli and Garnier, 2005; Paoli et al., 2008), their spreading and downwash (Scorer and Davenport, 1970) with the wake vortices forming behind aircraft at time scales of $1 \mathrm{~min}$ to $20 \mathrm{~min}$ (Lewellen and Lewellen, 2001) and their transition into widespread cirrus clouds and final decay at timescales of less than a hour to possibly days (Unterstrasser and Gierens, 2010a), are difficult to compute in one model. Three-dimensional (3d) large eddy simulation (LES) models resolve the fluid dynamics of wake vortex formation and decay (Gerz and Ehret, 1996; Lewellen and Lewellen, 1996; Holzäpfel et al., 2010; Misaka et al., 2012) and the bulk microphysics of contrails (Gierens, 1996; Chlond, 1998; Lewellen and Lewellen, 2001; Unterstrasser and Sölch, 2010; Naiman et al., 2011), but require large computing times. Even two-dimensional (2d) variants of such models, which allow for parameter studies and several hours of contrail ages, are too expensive to be applied for simulations of a large ensemble of contrails with realistic meteorological variability (Jensen et al., 1998a; Gierens and Jensen, 1998; Unterstrasser and Gierens, 2010b). Hence, simpler models with parameterized physics are required, providing proper results for the whole contrail life-cycle and for the global aircraft fleet under realistic meteorological conditions, with far less computing time.

Such a model, the "Contrail Cirrus Prediction Tool" (Co$\mathrm{CiP}$ ), is described in this paper. The model principles and its application for comparison with satellite data have been presented briefly before (Schumann, 2009). Basic model fea- tures and comparisons to in-situ observations have been published in Voigt et al. (2010). The model has been applied to estimate the global climate impact of contrail cirrus and to demonstrate the potential of mitigation of contrail climate impact by route optimization (Schumann et al., 2011a). This paper describes the details of the basic model concept, presents global results for illustration, and compares results for a few special cases with other models and observations.

\section{Model}

\subsection{The Gaussian concentration profile}

The spatial distribution of concentrations in a plume or contrail segment (without or with ice) at any time $t$ has to be know for computing plume properties depending on its width, depth, cross-section area, and shear-induced inclination. For this purpose, the concentration field in the plume is approximated as a Gaussian function of given width (or breadth) $B$, depth $D$, and inclination using the analytical relationships derived by Konopka (1995). Here, we describe the concentration profile. The computation of the plume parameters and their integration in time are explained in the following sections.

The concentration $c$ of a species per air mass in the plume with local orthogonal coordinates $(x, y, z)$ relative to the plume axis ( $x=$ flight direction, $y=$ cross-direction, $z=$ vertical) is approximated in the plane perpendicular to the segment axis by a Gaussian function of the position vector

$$
\begin{aligned}
& \mathbf{x}=(y, z)^{T}, \\
& c(\mathbf{x})=\frac{C_{0}}{A} \exp \left[-\frac{1}{2} \mathbf{x}^{T} \sigma^{-1} \mathbf{x}\right]
\end{aligned}
$$

The effective cross section area $A$ of the plume follows from the integral over all $y$ and $z$,

$$
A \equiv \iint \exp \left[-\frac{1}{2} \mathbf{x}^{T} \sigma^{-1} \mathbf{x}\right] d \mathbf{x} .
$$

As a consequence, see Appendix A1,

$A=2 \pi[\operatorname{det}(\sigma)]^{1 / 2}$,

and

$\iint c(\mathbf{x}) d \mathbf{x}=C_{0}$,

where $C_{0}(x, t)$ is the mass of the species per plume length and $C_{0} / A$ is the volume specific concentration in the center of the plume. Here, $\sigma(\mathbf{x}, t)$ is the covariance matrix of the concentration field $c$ in the plane for unit mass content $\left(C_{0}=\right.$ 1) (Konopka, 1995),

$\sigma=\iint(\mathbf{x} \otimes \mathbf{x}) c(\mathbf{x}) d \mathbf{x}$, 


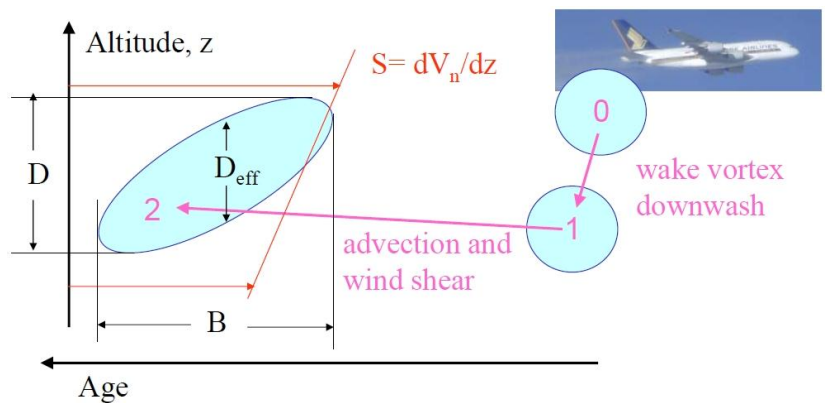

Fig. 1. Schematic of contrail dynamics versus altitude and contrail age with plume depth $D$, breadth $B$, and normal velocity shear $d V_{n} / d z$ at stages 0 : contrail formation, 1: downwashed wake vortex, 2: aged Gaussian cross-section.

(with dyadic product $\otimes$ ). The components of $\sigma$ describe a real symmetric and positive definite matrix,

$\sigma=\left(\begin{array}{cc}\sigma_{y y} & \sigma_{y z} \\ \sigma_{y z} & \sigma_{z z}\end{array}\right)$.

The inverse matrix is

$\sigma^{-1}=\frac{1}{\operatorname{det}(\sigma)}\left(\begin{array}{c}\sigma_{z z}-\sigma_{y z} \\ -\sigma_{y z} \sigma_{y y}\end{array}\right)$,

with $\operatorname{det}(\sigma)=\sigma_{y y} \sigma_{z z}-\left(\sigma_{y z}\right)^{2}$.

Since the area of an ellipse in normal form with width $B$ and depth $D$ is $A=\pi B D / 4$, we identify (see Fig. 1)

$B^{2}=8 \sigma_{y y}, D^{2}=8 \sigma_{z z}$.

For optical depth computation, as we will see, we need the effective vertical depth $D_{\text {eff }}=A / B$ which equals $D \pi / 4$ initially but gets smaller when the cross-section deforms with ambient shear.

This plume model has been used in the past to derive turbulent diffusivities from observed trace gas measurements and large eddy simulations (Schumann et al., 1995; Dürbeck and Gerz, 1995, 1996; Schlager et al., 1997), has been applied for air chemistry, sometimes as part of regional and global models (Schumann and Konopka, 1994; Karol et al., 1997; Kraabøl et al., 2000), and has been used as benchmark for tests of other models (Naiman et al., 2010; Unterstrasser and Gierens, 2010a). Compared to earlier plume studies (Danilin et al., 1994; Kärcher, 1995), this model accounts not only for turbulent diffusion but also shear. Its main advantage compared to an elliptical model described by Naiman et al. (2010) lies in the fact that the Konopka-model gives the exact solution of the advection-diffusion equations for constant shear and constant diffusivities.

The shape of aged contrails is sometimes not too far different from a Gaussian plume shape (Freudenthaler et al., 1995;

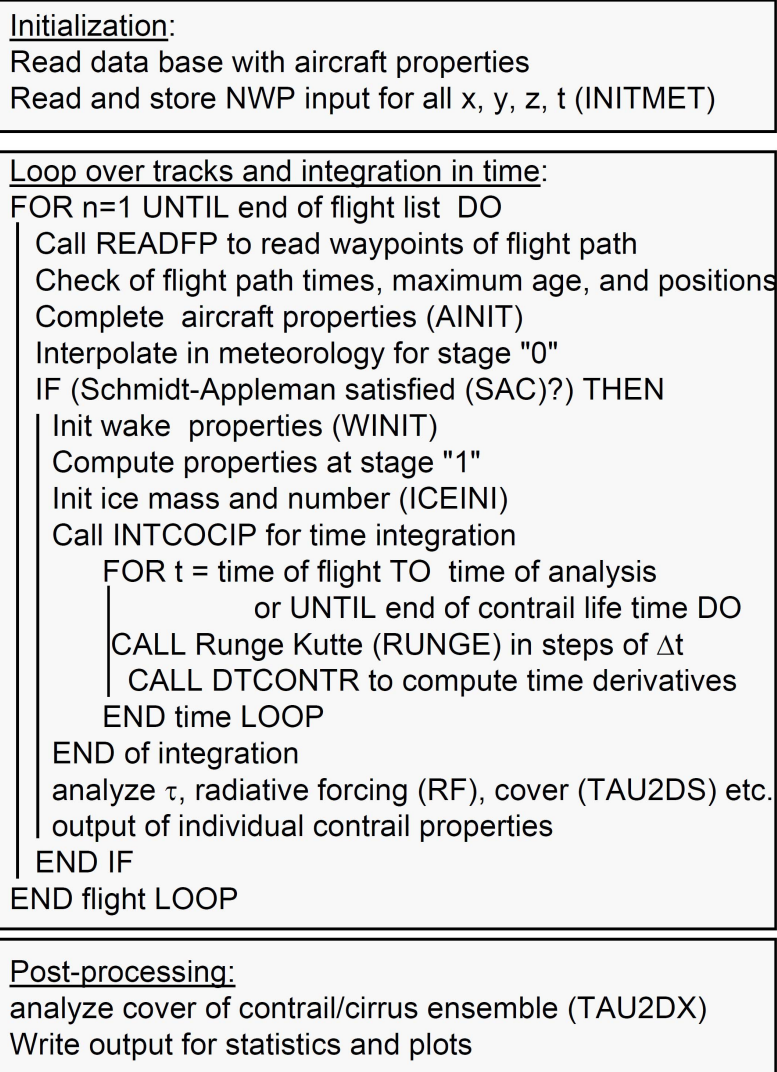

Fig. 2. CoCiP simulation framework. For a list of CoCiP modules, see Table 1.

Paugam et al., 2010). However, the initial exhaust jet, wake vortex and contrail (with primary and secondary parts) often deviate considerably from this shape (Sussmann and Gierens, 1999; Gerz et al., 1998; Holzäpfel et al., 2010; Lewellen and Lewellen, 2001). However, the Gaussian approximation allows for efficient simulation of global contrail cover.

\subsection{Numerical weather prediction input}

CoCiP simulates the contrails formed for given meteorology regionally or globally. Numerical weather prediction (NWP) data are used to determine the ambient meteorological conditions by linear interpolation for given positions and times (see Appendix A2). (For a list of abbreviations and frequently used symbols, see Tables A1 and A2 in the Appendix.)

Sub-module INITMET, see Fig. 2, reads input of discrete $3 \mathrm{~d}$ and time-dependent NWP fields for pressure $p$, geopotential altitude $z$, horizontal velocities $u$ (eastward) and $v$ (northward), pressure change rate $\omega=d p / d t$, absolute temperature $T$, absolute humidity $q$, cirrus ice water mass fraction $I_{\mathrm{C}}$, fractional cloud cover $C_{\mathrm{C}}$, and kinetic energy of subgridscale motions $E_{S G S}$. For analysis of radiative forcing, we also require input for the flux values of outgoing longwave 
radiation (OLR), the reflected solar radiation (RSR), and the solar direct radiation (SDR) at top of the atmosphere as a function of $x, y$, and $t$ from the NWP model. Finally, we specify the solar constant $S_{0}(t)$ for the time of the year consistent with the NWP data.

For global studies, we use analysis data from the Integrated Forecast System (IFS) of the European Center for Medium Range Weather Forecasts (ECMWF; see http:// www.ecmwf.int/research/ifsdocs/). Since a few years, the ECMWF model allows for supersaturation in clear air which has been demonstrated useful for this purpose (Tompkins et al., 2007; Rädel and Shine, 2007; Haywood et al., 2009; Lamquin et al., 2009, 2012). The model assumes that ice forms in a fraction of a grid cell when the supersaturation reaches the limit for homogeneous ice nucleation (Koop et al., 2000), typically of the order $150 \%$. In this study, the grid-cell mean humidity is taken as representative in both the cloudy and clear parts of the grid cell. For regional applications, we used NWP input from the COSMO-DE model of the German Weather Service (Baldauf et al., 2011). Also the meteorological fields from a global circulation climate model can be used to drive CoCiP.

Presumably the most critical input from the NWP model is the relative humidity RHi over ice, which is the dominant parameter for controlling the ice mass content in contrails (Unterstrasser and Gierens, 2010a). Contrails like cirrus, once formed, persist when the relative humidity is above a critical value $\mathrm{RHi}_{\mathrm{c}}$. In principle, $\mathrm{RHi}_{\mathrm{c}}=1$. However, supersaturated regions are shallow and narrow and, hence, may not be resolved by the discrete humidity field on a finite grid. Moreover, subgrid scale variability could cause local supersaturation in a grid cell that is subsaturated on average (Lamquin et al., 2009). Hence, the critical value $\mathrm{RHi}_{\mathrm{c}}$ is usually taken different and below $100 \%$ in NWP models. In the ECMWF model, this value is

$\mathrm{RHi}_{\mathrm{c}}=0.8$

in the mid-troposphere, 1.0 in the stratosphere and follows a smooth transition with pressure altitude between these two values in the upper $20 \%$ of the troposphere. For simplicity of further analysis, we divide the input value of $q$ by $\mathrm{RHi}_{\mathrm{c}}$ initially. Care has to be taken when interpolating humidity (see Sect. A2).

\subsection{Flight track and aircraft definition}

CoCiP simulates the contrails formed by cruising aircraft, flight by flight. Flight routes are prescribed on input as a sequence of waypoints versus flight time. For example, waypoint data from commercial flights over North America and Southern Canada are available in the internet (Garber et al., 2005). We got such data for case studies for German airspace from the Deutsche Flugsicherung (DFS) for a few days in October-November 2008, and for the European and the North Atlantic air space from EUROCONTROL for some days in August 2005. A global data set was setup by the United States (US) Federal Aviation Administration (FAA) with support from Volpe National Transportation Systems Center based on a cooperation by FAA, EUROCONTROL, and ICAO for 2006 (Wilkerson et al., 2010). For other periods, we simulate waypoint tracks for global commercial aviation using aircraft type, airport-connection, and schedule information from the Official Airline Guide (OAG), a commercially available product.

From such data, for each flight, a sub-module READFP reads the input to define the aircraft type and a list of $N_{W}>$ 1 waypoints $W_{i}=\left(x_{i}, y_{i}, z_{i}, t_{i}\right), i=1,2, \ldots, N_{W}$, in space and time, specifying longitude $x$, latitude $y$, altitude $z$, and time $t$, from start to end of the given flight track. The waypoints usually list the flight level $z_{i}$ which is converted to a static pressure $p_{0, i}$ according to the standard atmosphere of aviation (ICAO, 1964), see Appendix A4. CoCiP loops over the waypoints or over flight segments $S_{i}=\left(W_{i}, W_{i+1}\right)$, $i=1,2, \ldots, N_{W}-1$. For each waypoint, we set a flag to identify if the waypoint is followed by a contrail segment or a flight segment without contrail. This allows considering tracks with several non-consecutive contrails.

Wake vortices, particle formation, and the initial contrail dimensions depend obviously on aircraft and engine properties (Holzäpfel and Gerz, 1999; Sussmann and Gierens, 2001; Lewellen and Lewellen, 2001; Kärcher and Yu, 2009; Voigt et al., 2010; Naiman et al., 2011). This model accounts for the aircraft wing span $s_{\mathrm{a}}$, aircraft mass $M_{\mathrm{a}}$, true air speed $V_{\mathrm{a}}$, fuel consumption per flight distance $m_{F}$, soot number emission index $\mathrm{EI}_{\text {soot }}$, and the overall propulsion efficiency $\eta$. This efficiency is needed for deciding on contrail formation (Sect. 2.4) and defined as $\eta=F_{\mathrm{a}} /\left(m_{F} Q_{\text {fuel }}\right)$ as a function of thrust of engines or drag of aircraft $F_{\mathrm{a}}$, fuel flow per flight distance $m_{F}$, and fuel combustion heat $Q_{\text {fuel }}$ (Schumann, 1996). The soot emission index belongs to the critical model parameters, listed in Table 2. Aircraft usually burn kerosene with water vapor emission index $\mathrm{EI}_{\mathrm{H}_{2} \mathrm{O}}=1.23$ and combustion heat $Q_{\text {fuel }}=43.2 \mathrm{MJ} \mathrm{kg}^{-1}$. Other fuels can be simulated as well, e.g. $\mathrm{EI}_{\mathrm{H}_{2} \mathrm{O}}=8.94$ and $Q_{\text {fuel }}=120 \mathrm{MJ} \mathrm{kg}^{-1}$ for liquid hydrogen (Schumann, 1996).

Type dependent properties are set in module AINIT. The aircraft and operations data are collected from several sources, such as the BADA data set (EUROCONTROL, 2009) and soot emissions estimated within the AERO2K project (Eyers et al., 2004).

\subsection{Contrail formation conditions}

Each flight segment between consecutive waypoints on which contrails can form is treated as a contrail segment. We use the well-known Schmidt-Appleman criterion (SAC) (Schmidt, 1941; Appleman, 1953; Schumann, 1996). The SAC requires liquid saturation to occur locally in the plume of aircraft exhaust gases mixing with cold ambient air. The maximum threshold temperature $T_{\mathrm{LM}}$ is reached when the 
Table 1. Modules, in sequence of calls.

\begin{tabular}{lll}
\hline Name & Purpose & Sect. \\
\hline INITMET & meteorological fields & 2.2 \\
READFP & reads flight plans & 2.3 \\
AINIT & aircraft properties & 2.3 \\
SAC & Schmidt-Appleman criterion & 2.4 \\
WINIT & wake vortex & 2.5 \\
ICEINI & ice mass and number initialization & $2.6-2.7$ \\
INTCOCIP & integration over several time steps & 2.8 \\
RUNGE & Runge-Kutta & 2.8 \\
DTCONTR & time derivatives and contrail properties & $2.8-2.12$ \\
RADI & radiative forcing analysis & 2.14 \\
TAU2DS & cloud mask for one contrail & 2.15 \\
TAU2DX & cloud mask for cirrus & 2.15 \\
\hline
\end{tabular}

relative humidity over liquid water in ambient air is at saturation, $U=1, U=$ RHi $p_{\text {ice }}(T) / p_{\text {liq }}(T)$. The saturation pressures over liquid and over ice water surfaces, $p_{\text {liq }}$ and $p_{\text {ice, }}$, are computed as in Sonntag (1994), see Appendix A3. An often used approximation for $T_{\mathrm{LM}}$ (liquid maximum) is

$T_{\mathrm{LM}}=-46.46+9.43 \ln (G-0.053)+0.72[\ln (G-0.053)]^{2}$,

with $G$ in units of $\mathrm{Pa} \mathrm{K}^{-1}$ and $T_{\mathrm{LM}}$ in ${ }^{\circ} \mathrm{C}$ (Schumann, 1996), and with errors below $0.07 \mathrm{~K}$ for $0.24 \mathrm{~Pa} \mathrm{~K}^{-1}<$ $G<23 \mathrm{~Pa} \mathrm{~K}^{-1}$. Here, $G$ is the steepness of the mixing line between engine exit and ambient air in a $p-T$ diagram,

$G=\frac{c_{p} p \mathrm{EI}_{\mathrm{H}_{2} \mathrm{O}}}{\left(M_{\mathrm{H}_{2} \mathrm{O}} / M_{\text {air }}\right) Q_{\text {fuel }}(1-\eta)}$,

with specific heat capacity of air $c_{p}=1004 \mathrm{~J}(\mathrm{~kg} \mathrm{~K})^{-1}$, ratio of molecular masses of water and air $M_{\mathrm{H}_{2} \mathrm{O}} / M_{\text {air }}=0.622$, pressure $p$, water emission index $\mathrm{EI}_{\mathrm{H}_{2} \mathrm{O}}$ and combustion heat $Q_{\text {fuel }}$ of the fuel used, and overall propulsion efficiency $\eta$ of the aircraft at cruise. All these values are available for analysis within CoCiP. For kerosene-driven aircraft, at $p=(100-$ $500) \mathrm{hPa}, G$ varies typically within $(0.6-4) \mathrm{Pa} \mathrm{K}^{-1}$. Larger values occur for fuels with larger water mass contents.

So far, no such approximation was available for the threshold temperature $T_{\mathrm{LC}}$ at $0<U<1$. Since $T_{\mathrm{LC}}$ has to be computed for each waypoint, we developed a new efficient approximation as presented in Appendix A5.

An alternative contrail threshold criterion avoiding iterative solutions for given $T_{\mathrm{LM}}$ has been suggested by Ponater et al. (2002). Instead of $T<T_{\mathrm{LC}}$, it requires that the ambient relative humidity $U$ stays above a critical value

$U_{\mathrm{LC}}=\frac{G\left(T-T_{\mathrm{LM}}\right)+p_{\mathrm{liq}}\left(T_{\mathrm{LM}}\right)}{p_{\text {liq }}}$,

or correspondingly $\mathrm{RHi}>\mathrm{RHi}_{\mathrm{LC}}$. This more efficient variant is used unless $T_{\mathrm{LC}}$ is required for analysis.
An explicit criterion for persistency as a function of supersaturation is not necessary in CoCiP. In case of very low temperatures, short-lived contrails may form from the emitted water vapor even in totally dry air. Contrails are sometimes observed in slightly subsaturated air masses (Schröder et al., 2000; Rädel and Shine, 2007; Krämer et al., 2009; Voigt et al., 2010). At least at low temperatures, it may take up to an hour until the ice formed initially from emitted water is sublimated, see Appendix A6. Contrails in subsaturated air masses reach small cover and, hence, contribute little to radiative forcing (Ponater et al., 1996). Nevertheless, such contrails may be important when comparing contrail properties to observations (Voigt et al., 2011). Also, soot properties may possibly get changed when processed in contrails (Lee et al., 2010).

\subsection{Wake vortex downwash}

Since the global climate impact of contrails comes mainly from contrails surviving far longer than the wake vortex phase, we do not resolve the details of the jet and wake dynamics in the first minutes. Instead, we use a parametric model to estimate the initial depth $D_{0}$, width $B_{0}$, and maximum and mean downward displacements $\Delta z_{w}$ and $\Delta z_{1}$ such that the follow-on advection and dispersion is approximately consistent with the results of the Gaussian model. After formation of the contrail in the jet of the aircraft engines at point "0", see Fig. 1, the sinking of the contrail with the wake vortex behind the aircraft is estimated using a wake vortex model. The subsequent computations then start from model point " 1 " at the time and horizontal position of the contrail forming aircraft but at lower altitude, see Fig. 1. The plume model assumes that aircraft wake induced turbulence affecting the initial plume dimensions has ceased at this stage.

A wake vortex sub-model WINIT computes the maximum downward displacement $\Delta z_{w}$ of the contrails at the end of the wake vortex phase as a function of aircraft and atmospheric parameters using a parameterization derived from a nondimensional fit to results of the Probabilistic Two-Phase Aircraft Wake-Vortex Model (P2P) (Holzäpfel, 2003). The sinking and decaying wake vortex depends on typical length and time scales of the aircraft, ambient stratification, and ambient turbulence which are defined as:

$$
\begin{aligned}
& \text { wake vortex separation } b_{0}=\pi s_{\mathrm{a}} / 4, \\
& \text { initial circulation } \Gamma_{0}=4 M_{\mathrm{a}} g /\left(\pi s_{\mathrm{a}} \rho V_{\mathrm{a}}\right), \\
& \text { effective time scale } t_{0}=2 \pi b_{0}^{2} / \Gamma_{0}, \\
& \text { initial velocity scale } w_{0}=\Gamma_{0} /\left(2 \pi b_{0}\right), \\
& \text { and normalized dissipation rate } \epsilon *=\left(\epsilon b_{0}\right)^{1 / 3} / w_{0}, \\
& \text { which are functions of wing span } s_{\mathrm{a}}, \\
& \text { aircraft mass } M_{\mathrm{a}},
\end{aligned}
$$




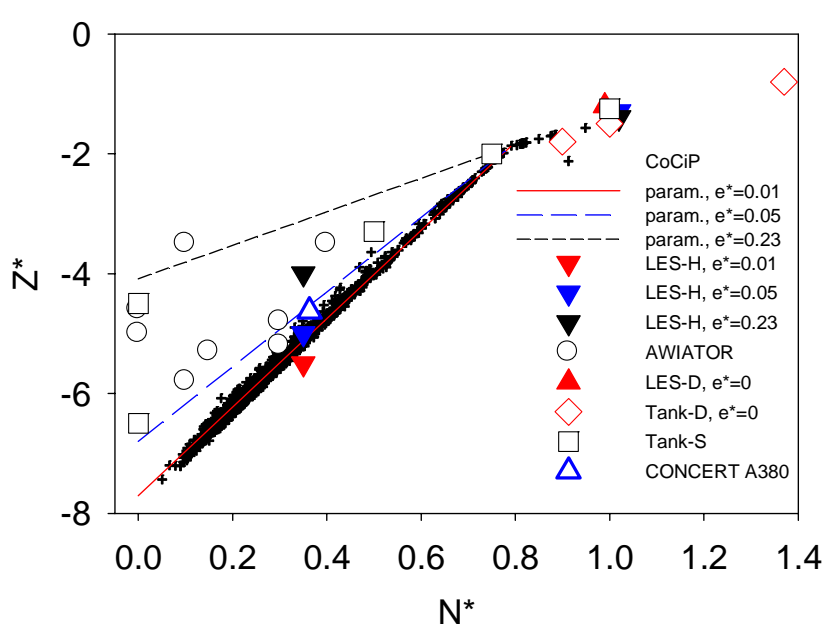

Fig. 3. Non-dimensional maximum wake vortex sinking distance versus nondimensional stratification. $Z *=-\Delta z_{w} / b_{0}, b_{0}=$ $(\pi / 4) s_{\mathrm{a}}, \quad N *=N_{\mathrm{BV}} t_{0}, \quad t_{0}=2 \pi b_{0}^{2} / \Gamma_{0}, \quad \Gamma_{0}=M_{\mathrm{a}} g 4 /\left(\pi s_{\mathrm{a}} \rho V_{\mathrm{a}}\right)$. Crosses depict CoCiP results. The deviation from a piece-wise straight line is a consequence of different (non-dimensional) turbulent dissipation rates $\epsilon *=\left(\epsilon b_{0}\right)^{1 / 3} / w_{0}, w_{0}=b_{0} / t_{0}$. The lines depict the parameterization for fixed $\epsilon *=0.01,0.05$ and 0.23 (red, blue, black) (Holzäpfel, 2003). Filled symbols with corresponding colors are LES results (Hennemann, 2010; Delisi and Robins, 2000). Open symbols are experimental results in tanks (Sarpkaya, 1983; Delisi and Robins, 2000) and behind aircraft (deBruin and Kannemans, 2004; Voigt et al., 2011) (projects AWIATOR and CONCERT).

true air speed $V_{\mathrm{a}}$,

air density $\rho$,

Brunt-Vaisaila frequency $N_{\mathrm{BV}}$, and turbulent kinetic energy dissipation rate $\epsilon$.

The parameterization distinguishes between strongly and weakly stably stratified conditions: If $N_{\mathrm{BV}} t_{0} \geq 0.8$ :

$\Delta z_{w}=1.49 \frac{w_{0}}{N_{\mathrm{BV}}}$,

else, with $\epsilon * \leq 0.36$,

$\frac{\Delta z_{w}}{b_{0}}=7.68\left(1-4.07 \epsilon *+5.67 \epsilon *^{2}\right)\left(0.79-N_{\mathrm{BV}} t_{0}\right)+1.88$.

The maximum sinking $\Delta z_{w}$ is larger than the value $w_{0} / N_{\mathrm{BV}}$ for a pure Brunt-Vaisaila oscillation because of additional rotational momentum in the sinking and rotating vortices (Holzäpfel and Gerz, 1999). The influence of ambient turbulence on wake vortex decay enters this model as a function of the turbulent dissipation rate $\epsilon$ in the atmosphere. As discussed later, the value of this parameter may vary in the range $\left(10^{-8}-10^{-2}\right) \mathrm{m}^{2} \mathrm{~s}^{-3}$ (Schumann et al., 1995; Gultepe and Starr, 1995). For small dissipation rate, the downwash depth depends only weakly on its value. Here we use

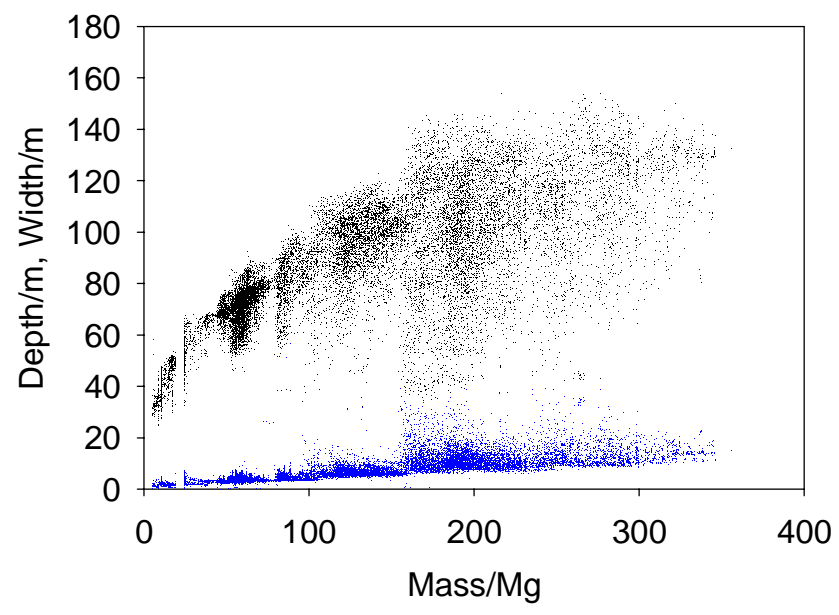

Fig. 4. Initial contrail depth (black) and width (blue) versus aircraft mass for an example traffic and meteorology over the North Atlantic 6-9 June 2006. Each point corresponds to an aircraft at a given position. Following the International System of Units (NIST, 2008), non-dimensional axis values (here: mass/Mg) represent the quantity plotted (mass) divided by the given unit (Mg).

Eq. (37), see below, to estimate dissipation as a function of ambient shear. Typical values are of the order $10^{-5} \mathrm{~m}^{2} \mathrm{~s}^{-3}$.

The wake vortex reaches maximum downward displacement at times which are 5-12 times larger than $t_{0}$, depending on stratification (Holzäpfel, 2003). Hence, $\Delta z_{w}$ is rarely observed in the atmosphere because this occurs up to $50 \mathrm{~km}$ behind the aircraft and the measurable wake turbulence is weak at this stage. Nevertheless, the empirical fit is consistent with the few existing field and laboratory results, see Fig. 3. This figure contains previously published results and, in addition, the result computed with the present approximation for one of the largest commercial aircraft (A380) as observed during the CONCERT campaign (Voigt et al., 2010, 2011 ), with $V_{\mathrm{a}}=250 \mathrm{~m} \mathrm{~s}^{-1}, M_{\mathrm{a}}=508 \mathrm{Mg}, s_{\mathrm{a}}=79.8 \mathrm{~m}, \rho=$ $0.39 \mathrm{~kg} \mathrm{~m}^{-3}, N_{\mathrm{BV}}=0.012 \mathrm{~s}^{-1}, \epsilon=10^{-5} \mathrm{~m}^{3} \mathrm{~s}^{-2}$. Hence, $b_{0}=62.7 \mathrm{~m}, t_{0}=30.3 \mathrm{~s}, w_{0}=2.07 \mathrm{~m} \mathrm{~s}^{-1}, N *=N_{\mathrm{BV}} t_{0}=$ $0.363, \epsilon *=\left(\epsilon b_{0}\right)^{1 / 3} / w_{0}=0.04$. With these parameters, the above model computes a maximum wake vortex sinking of $Z *=-\Delta z_{w} / b_{0}=-4.63$ (see Fig. 3) or $\Delta z_{w}=290 \mathrm{~m}$. This value is close to the observed value of $270 \mathrm{~m}$.

The initial sinking from state " 0 " to "1" (Fig. 1) is set to

$\Delta z_{1}=C_{z 1} \Delta z_{w}, C_{z 1}=0.25$.

The center of the contrail is assumed to start higher than midway between the initial and maximum sinking distance, partly because of buoyancy. The initial contrail depth $D_{1}$ is set to

$D_{1}=C_{D 0} \Delta z_{w}, C_{D 0}=0.5$.

The initial contrail depth is taken considerably smaller than $\Delta z_{w}$, because initial tests have shown that otherwise the 
model simulates too large dilution compared to observations. In fact, the initial contrail width $B_{1}$ is parameterized,

$B_{1}=N_{\mathrm{dil}}\left(t_{0}\right) m_{F} /\left[(\pi / 4) \rho D_{1}\right]$,

so that the dilution $N_{\text {dil }}$ at the time $t=t_{0}$ of wake vortex formation fits an often used empirical function

$N_{\text {dil }}(t) \approx 7000\left(t / t_{\mathrm{s}}\right)^{0.8}$,

with $t_{\mathrm{S}}=1 \mathrm{~s}$ (Schumann et al., 1998).

For a fleet of aircraft and typical meteorological conditions, Fig. 4 illustrates the initial depth and width results. These scales increase with aircraft mass, as expected. For the same aircraft mass, the initial depth is largest for weak stratification. The initial width is usually far smaller than the depth.

\subsection{Initial contrail ice crystal mass concentration}

We simulate the contrail ice properties using two plume-bulk ice quantities, the mass mixing ratio $I$ of ice in the contrail, and the total number concentration $N$ of contrail ice particles per contrail length. We prescribe $N$ and not the volume specific ice number concentration $n=N / A$ because $N$ is conserved during plume mixing.

We assume that the plume vapor is at ice saturation within the effective cross-section area $A$ (Eq. 3) of the contrail plume at all times. Besides for young contrails with low ice mass and low particle concentrations, this assumption is supported for contrails after the vortex formation even for large ambient supersaturation by large eddy simulations (Lewellen and Lewellen, 2001; Unterstrasser and Gierens, 2010a; Paugam et al., 2010) and measurements (Heymsfield et al., 1998). The temperature increase in the plume from combustion heat and later by sublimation heat is small after a few seconds of contrail age, see Appendix A6. Hence, the contrails are assumed to be in thermal equilibrium with ambient air in the wake phase after contrail formation. These assumptions are essential for efficient simulation. As a consequence, the ice mass content $I$ can be computed from pure thermodynamics. Otherwise, we would have to solve also a budget equation for heat, and the resolution of vertical oscillations in stratified air would require time steps smaller than $N_{\mathrm{BV}}^{-1}$.

The initial value $I_{0}$ at stage " 0 " is computed in a module ICEINI as the sum of the water mass emitted by the engines from burning kerosene mass per flight distance plus the water mass concentration available from humidity in the air mass entrained into the young contrail:

$I_{0}=\frac{\mathrm{EI}_{\mathrm{H}_{2} \mathrm{O}} m_{F}}{(\pi / 4) \rho D_{1} B_{1}}+q_{0}-q_{\mathrm{s}}\left(p_{0}, T_{0}\right)$.

The first term is the water mass emitted by the engines from burning kerosene per flight distance. The last two terms measure the amount of water vapor above ice saturation available in the air mass entrained into the young contrail. This amount is deposited on ice particles at ice saturation. Here, $q_{0}$ is the ambient humidity (mass ratio) and $q_{\mathrm{s}}(p, T)=$ $\left(R_{0} / R_{1}\right) p_{\text {ice }}(T) / p$ is the saturation humidity at point " 0 ". $I_{0}$ is replaced by zero if becoming negative (in dry air), and this ends the contrail life-cycle, for such cases.

During sinking of the contrail with the wake vortex, part of the initial ice mass sublimates because of adiabatic warming (Holzäpfel and Gerz, 1999; Sussmann and Gierens, 1999; Lewellen and Lewellen, 2001). Hence, the ice water mass fraction $I_{1}$ in the contrail at stage " 1 " (at the end of the wake vortex phase) is smaller:

$I_{1}=I_{0}-\Delta I_{\mathrm{ad}}$

where

$\Delta I_{\text {ad }}=\frac{R_{0}}{R_{1}}\left[\frac{p_{\text {ice }}\left(T_{0}+\Delta T_{\mathrm{ad}}\right)}{p_{1}}-\frac{p_{\text {ice }}\left(T_{0}\right)}{p_{0}}\right]$

and

$\Delta T_{\mathrm{ad}}=T_{0}\left(R_{0} / c_{p}\right)\left(p_{1}-p_{0}\right) / p_{0}$,

with gas constant $R_{0}$ and specific heat capacity $c_{p}$ of air. Again, the contrail life-cycle ends if the above expression gives a negative result (in dry air). Without aircraft emissions, a plume starting at $p_{0}=250 \mathrm{hPa}, T_{0}=220 \mathrm{~K}$, with $\mathrm{RHi}=1.5$ has to descend $400 \mathrm{~m}$ according to these equations to reach saturation $(\mathrm{RHi}=1)$, in agreement with earlier estimates (Lewellen and Lewellen, 2001; Unterstrasser et al., 2008).

\subsection{Initial contrail ice crystal number concentration}

The initial number of ice particles is a result of liquid droplets which form by nucleation on emitted and ambient aerosols and which freeze shortly thereafter (Kärcher et al., 1996; Fahey et al., 1999). The local relative humidity at the place of contrail formation in the exhaust plume exceeds liquid saturation, so that liquid droplets form at least on the larger soot particles in the young contrail, which then freeze quickly because they are relatively large (compared to volatiles), and form at low temperature and high relative humidity in the contrail. This nucleation process depends on aerosol properties, mixing, and local temperature and humidity in the plume. This process has been extensively studied with measurements and models (Busen and Schumann, 1995; Schumann et al., 1996; Kärcher et al., 1996; Brown et al., 1997; Jensen et al., 1998b; Kärcher, 1998; Kärcher et al., 1998; Schumann et al., 2002; Paoli et al., 2008; Wong and MiakeLye, 2010). Ice particle formation is a self-limiting process in which vapor depletion by the first ice particles limit further nucleation (Jensen et al., 1998b; Kärcher et al., 1998). The initial number of ice particles is essentially determined by the number of soot particles emitted by the engines (Brown et al., 1997; Kärcher, 1998). In principle, also nucleation in volatile 
materials, e.g. sulfuric acid and organic material, contributes to ice formation, however, the contribution of volatiles is small for typical soot emissions and moderately low temperatures (Kärcher and Yu, 2009). The few existing measurements of ice and soot particle concentrations in young contrails are hardly accurate enough to test this assumption (Schumann et al., 2002). However, analysis of experiments for different fuel sulfur contents show that the number of ice particles in young contrails increases by only about a factor 1.3 for an increase in fuel sulfur content from $6 \mu \mathrm{g} \mathrm{g}^{-1}$ to $2800 \mu \mathrm{g} \mathrm{g}^{-1}$ (Brown et al., 1997; Kärcher et al., 1998; Schumann et al., 2002). Volatile particles contribute a larger share to ice nucleation for low soot particle emissions (below $10^{14} \mathrm{~kg}^{-1}$ ) and low ambient temperatures (below $210 \mathrm{~K}$ ) (Kärcher and Yu, 2009).

Hence, the initial number $N_{0}$ of ice particles per contrail length in the freshly formed contrail at stage " 0 " is assumed to be determined by soot, and prescribed (in a module ICEINI) as a function of the soot emission index and the fuel consumption rate per flight distance,

$N_{0}=\mathrm{EI}_{\text {soot }} m_{F}$.

Contributions of ice particles from other sources, such as nucleation in volatiles or aerodynamic contrails (Gierens et al., 2009; Kärcher et al., 2009b), could be included when proper parameterizations become available.

Only a fraction of the ice crystals survives the transition between stages " 0 " and " 1 ". Ice particle loss by Brownianmotion-induced coagulation after the jet phase is small (Gierens, 1996; Kärcher, 1998; Paoli et al., 2008). However, important loss is caused in the sinking vortex (Sussmann and Gierens, 1999; Lewellen and Lewellen, 2001; Unterstrasser et al., 2008). Any initial supersaturation gets reduced by deposition of humidity on ice particles quickly and before the vortex has reached its lowest altitude. The sinking vortex with adiabatic heating causes local subsaturation around the ice particles. As a consequence, the ice particles sublimate and some of them disappear (Unterstrasser and Sölch, 2010). Also turbulent detrainment from the sinking wake vortex in dry air may contribute to particle losses. These losses depend on aircraft type, aircraft speed and mass, soot and heat emissions, humidity, temperature, shear, stratification, ambient turbulence, and particle sizes (Huebsch and Lewellen, 2006; Unterstrasser et al., 2008; Unterstrasser and Gierens, 2010a,b; Naiman et al., 2011).

Hence, we prescribe a "survival factor" $f_{\text {surv }}$ of ice particles remaining after the vortex phase:

$N_{1}=f_{\text {surv }} N_{0}$.

As a first approximation, we assume that the surviving particle fraction is in line with ice mass changes,

$f_{\text {surv }}=I_{1} / I_{0}, 0<f_{\text {surv }}<1$.

Implicitly this is a function of ambient humidity and temperature. The survival factor varies between 1 and about 0.7 .

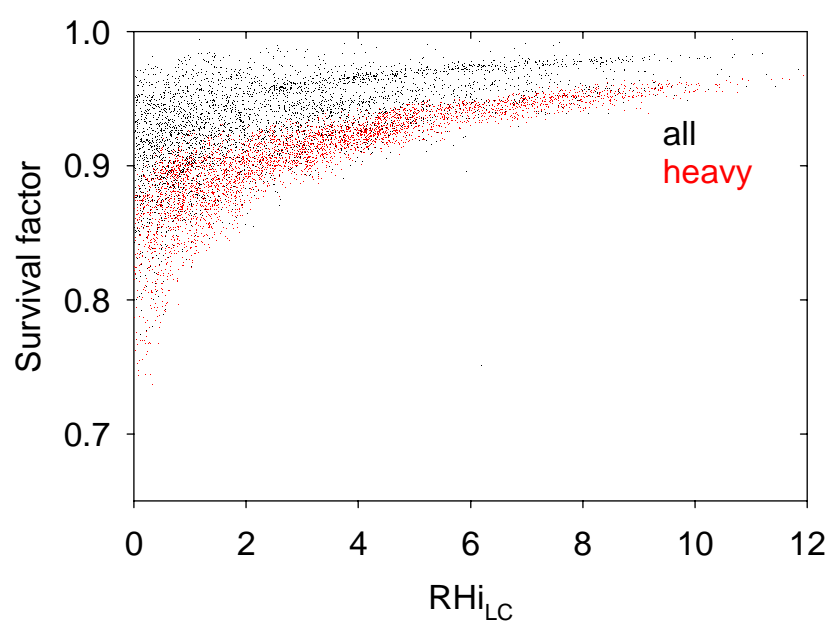

Fig. 5. Survival factor for ice mass due to adiabatic wake vortex sinking versus the critical relative humidity over ice $\mathrm{RHi}_{\mathrm{LC}}$ required for contrail formation, for meteorology over the North Atlantic for 6-9 June 2006. Each point corresponds to an aircraft with properties representing aircraft types in the traffic input (black) or with properties of an heavy aircraft (red).

LES models compute smaller factors (Unterstrasser et al., 2008; Kärcher et al., 2009a). The difference is partly caused by the small sinking distance $z_{1}$ of the bulk plume (in contrast to maximum sinking), see Eq. (13). In principle, ice mass and ice number evolve differently (Gierens and Bretl, 2009) implying different survival factors. Therefore, we cannot exclude at this stage that smaller survival factors might give better results. Anyway, the ratio $I_{1} / I_{0}$ is aircraft dependent. The survival factor depends on the temperature and humidity differences relative to the SAC threshold values, see Fig. 5, because the plume starts sinking with initial ice mass depending on these differences.

\subsection{Time integration and segment trajectories}

After contrail initialization at stage "1" slightly below the aircraft track, the contrail trajectories and properties are followed with the Gaussian plume model until a given time of analysis or until final disappearance of the contrail at any later stage "2" (Fig. 1). This evolution is computed by numerical integration of the Lagrangian contrail position and properties in a sub-module INTCOCIP. The integration in time $t$ is performed over a sequence of time steps $\Delta t$. For global simulations, we may use large time steps, of the order of $1 \mathrm{~h}$, for computational efficiency. The ice mass and particle concentrations have to stay non-negative. Hence, the integration scheme has to be accurate, positive definite, and unconditionally stable.

The state of the contrail is characterized by a state vector $X(t, i), i=1,2, \ldots, N_{W}$, containing $X=x, y, p, \sigma, I, N$, including horizontal positions, static pressure, plume parameters, mass specific ice mass content, and total number of ice 
particles per contrail length. All components of $X$ depend on position in space and time $t$. The integration is performed for each contrail segment between two successive contrail points.

For the trajectory part $X=(x, y, p)$ of the state vector, we could use the standard second-order two-step Runge-Kutta scheme (Abramowitz and Stegun, 1964). Lagrangian models for trajectory calculation in meteorology often use a first order or the two-step second order scheme, which is unconditionally stable for smooth wind fields, without iterations (Danilin et al., 1994; Nair et al., 2003; Stohl et al., 1998, 2001; Wernli and Davies, 1997). However, for accuracy at large time steps, and because of strong nonlinearities of other plume parameters, we apply the Runge-Kutta scheme with a few (2-6) iteration steps. Accuracy tests have shown that the time step $\Delta t$ does not need to be taken much smaller than the time step of the available NWP results. This is very important for the efficiency of the scheme.

The integration over one step is performed in a sub-module RUNGE. Time derivatives of $X$ are computed in module DTCONTR. For each time step, these routines internally assume that the input was given for a time point " 1 " and the integration ends after one step at a time " 2 ", with $\Delta t=t_{2}-t_{1}$.

The Runge-Kutta scheme starts with a predictor step:

$\tilde{X}=X(t)+\Delta t \frac{\partial}{\partial t} X(t)$,

followed by one or several corrector steps,

$X(t+\Delta t)=X(t)+\frac{\Delta t}{2}\left[\frac{\partial}{\partial t} X(t)+\frac{\partial}{\partial t} \tilde{X}\right]$,

$\tilde{X}:=X(t+\Delta t)$.

The time derivatives of the positions are computed using the wind vector $(U, V, \omega)$ and ice particle terminal fall speed $V_{T}$,

$\partial x / \partial t=U, \partial y / \partial t=V, \partial p / \partial t=\omega+g \rho V_{T}$.

Here, we assume that the contrail follows the mid point of the bulk of the ice particles under sedimentation so that the point "2" gets displaced downwards according to the terminal fall speed $V_{T}$. Vertical advection may be computed following kinematic or diabatic trajectories (Danielsen, 1961). The local heating rates required for the diabatic approach are not generally available from NWP output (Fueglistaler et al., 2009). Here, we use the kinematic variant for given $\omega$, although it may cause excessive vertical dispersion and noise for large time steps due to waves and assimilation errors (Schoeberl et al., 1997; Ploeger et al., 2002). Adiabatic advection for constant potential temperature was tested, but showed only minor changes in contrail statistics compared to the kinematic variant. The other components of $X$ are integrated quasi analytically as described below.

For numerical integration, one has to keep the way-point variables at the start of the time-step, at the intermediate level denoted with tilde, and at the end of the time step. Thereafter, older results may be forgotten, unless required for later analysis. For old contrail waypoints originating from the same aircraft at times before $t_{1}$, integration is performed with the given time step. For new contrail waypoints getting initiated within the time interval, a smaller time step is used to reach $t_{2}$. Polewards of $|y|>80^{\circ}$, these calculations are performed in Cartesian coordinates to avoid the pole singularity, see Appendix A7. This seems to be common practice in trajectory models (Nair et al., 2003), but details have not been published. Moreover, care is needed to make sure that the coordinates stay consecutive when passing the date line (at $\pm 180^{\circ}$ ) for periodicity in longitude (see Appendix A8).

It should be noted that advection with horizontally diverging wind fields changes the segment length $L$ between waypoints. Individual waypoints may depart from each other considerably over the time of integration, so that $L$ changes by factors of order 0.5-2. Because of continuity, horizontal divergence is connected with convergence in vertical planes and this reduces the cross-section area. The change in segment length $L(t)$ is taken into account when integrating cross-sections and the particle concentration $N$ per unit contrail length in time. Segment length is computed efficiently as square root of the sum of squared geographical coordinate differences (in meters), except in polar regions where great circle equations are used (http://www.astro.uu. $\mathrm{nl} / \sim$ strous/AA/en/reken/grootcirkel.html).

For illustration, Fig. 6 shows contrails analyzed for global traffic and for ECMWF NWP data for a time slice at an arbitrary analysis time 06:00 UTC 6 June 2006. This analysis includes contrails with maximum age of $<36 \mathrm{~h}$. The code actually reads NWP data for a 5-days period, including the period $36 \mathrm{~h}$ before the analysis time. The plot shows in red the contrails existing at the analysis time. In addition, the flight paths of aircraft cruising in cold and humid air causing at least short contrails are plotted for the 3-h time interval before the analysis time. Most of the contrails at analysis time originate from recent flights but some from flights that occurred more than one day (and up to $36 \mathrm{~h}$ ) before. Young contrails experience little advection, but older contrails get advected partly over large distances as indicated by the separation between red and black lines. Contrails occur in clusters in regions with supersaturation. Many of these regions also include natural cirrus. This coexistence of cirrus and contrails will be taken into account when analyzing the radiative forcing and contrail cirrus cover. The results will be discussed further below.

\subsection{Evolution of Gaussian plume parameters}

The initially symmetrical Gaussian contrail inclines and grows in cross-section area $A$ with time because of shear (vertical gradient of the horizontal velocity normal to the contrail axis), $S=d V_{n} / d z$, of either sign, and horizontal 


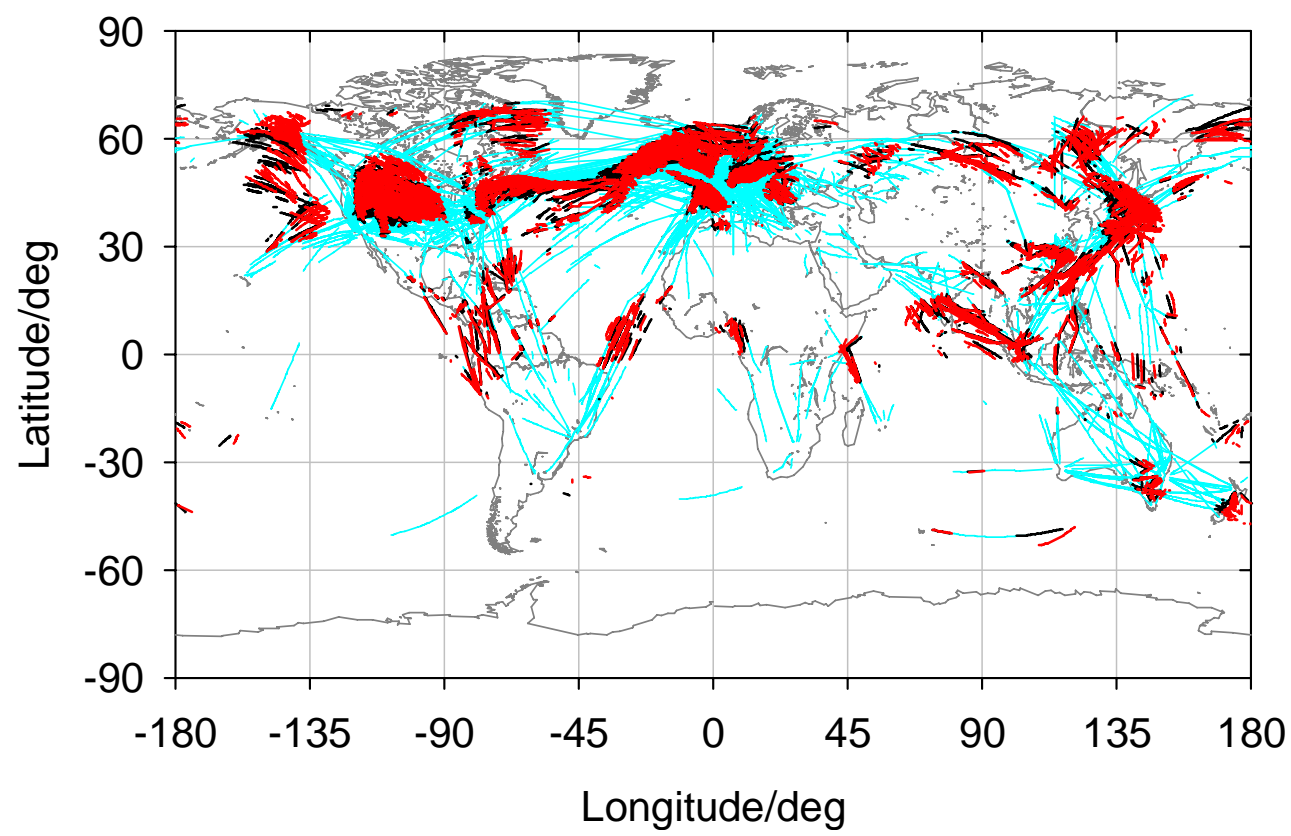

Fig. 6. Contrail forming flight paths (cyan) for global traffic between 03:00-06:00 UTC 6 June 2006. Contrails existing at 06:00 UTC that day are identified by red lines. Those parts of the tracks where these contrails originated are shown in black.

and vertical diffusivities, $D_{\mathrm{H}}$ and $D_{\mathrm{V}}$, and possibly shear diffusivity $D_{\mathrm{S}}$ (of either sign) with the constraint

$D_{\mathrm{S}}^{2} \leq D_{\mathrm{V}} D_{\mathrm{H}}$

for positiv definite solutions. As derived by Konopka (1995), with extension for variable segment length $L(t)$, the temporal evolution of $\sigma(t)$, for constant values of $S, D_{\mathrm{H}}, D_{\mathrm{V}}, D_{\mathrm{S}}$ follows from

$$
\begin{aligned}
& \sigma_{y y}(t+\Delta t)=\left[\frac{2}{3} S^{2} D_{\mathrm{V}} \Delta t^{3}+\left(S^{2} \sigma_{z z}(t)+2 D_{\mathrm{S}} S\right) \Delta t^{2}\right. \\
& \left.\quad+2\left(D_{\mathrm{H}}+S \sigma_{y z}(t)\right) \Delta t+\sigma_{y y}(t)\right][L(t) / L(t+\Delta t)]^{2} \\
& \sigma_{z z}(t+\Delta t)=2 D_{\mathrm{V}} \Delta t+\sigma_{z z}(t) \\
& \sigma_{y z}(t+\Delta t)=\left[S D_{\mathrm{V}} \Delta t^{2}\right. \\
& \left.+\left(2 D_{\mathrm{S}}+S \sigma_{z z}(t)\right) \Delta t+\sigma_{y z}(t)\right] L(t) / L(t+\Delta t) .
\end{aligned}
$$

The equations assume that only the horizontal plume scales change inversely to the segment length $L(t)$, while the vertical stays constant. The vertical to horizontal divergence ratio could be estimated from the NWP model, if necessary.

The contrail model starts from initial values $\sigma_{y y}\left(t=t_{0}\right)=$ $B^{2} / 8, \sigma_{z z}\left(t=t_{0}\right)=D^{2} / 8, \sigma_{y z}\left(t=t_{0}\right)=0$, see Eqs. $(14$, 15). Thereafter, these equations are used with equal-weight algebraic mean values of $D_{\mathrm{H}}, D_{\mathrm{V}}, D_{\mathrm{S}}$, and $S$ at times $t$ and $t+\Delta t$ to integrate over time to $t+\Delta t$. (The accuracy of the method might be improved by weighting the contributions differently.) Linear diffusivity changes with time could be treated analytically (Konopka, 1995), but any parameterization of the diffusivities as a function of plume scales makes the equations nonlinear. Hence, the solutions are no longer exact and the accuracy becomes time-step dependent. However, the integration is unconditionally stable and guarantees positive definite solutions of $\sigma$.

In the code we compute $A$ from Eq. (3) for each waypoint. However, the analytical solution for $A$ is

$$
\begin{gathered}
A(t+\Delta t)=2 \pi\left[\frac{1}{3} S^{2} D_{\mathrm{V}}^{2}(\Delta t)^{4}+\frac{2}{3} S^{2} D_{\mathrm{V}} \sigma_{z z}(t)(\Delta t)^{3}\right. \\
+\left(2 S D_{\mathrm{V}} \sigma_{z z}(t)-2 S D_{\mathrm{S}} \sigma_{z z}(t)+4 D_{\mathrm{H}} D_{\mathrm{V}}-4 D_{\mathrm{S}}^{2}\right)(\Delta t)^{2} \\
+\left(2 D_{\mathrm{V}} \sigma_{z z}(t)+2 D_{\mathrm{H}} \sigma_{z z}(t)-4 D_{\mathrm{S}} \sigma_{y z}(t)\right) \Delta t \\
\left.+\sigma_{y y}(t) \sigma_{z z}(t)-\sigma_{y z}^{2}(t)\right]^{1 / 2} .
\end{gathered}
$$

Except for a factor 2, Eq. (32) is the same as Eq. (9) of Dürbeck and Gerz (1996). It should be noted that shear alone does not increase the cross-section $A$. It makes the elliptical contrail cross-section wider but also thinner. But in combination with turbulent diffusion, mainly vertically, shear enhances mixing considerably. The vertical diffusivity is most important for growth, since when $D_{\mathrm{V}}=0\left(D_{\mathrm{S}}=0\right.$ because of Eq. 28), Eq. (32) reduces to $A(t+\Delta t)=2 \pi\left[2 D_{\mathrm{H}} \sigma_{z z} \Delta t+\right.$ $\left.\sigma_{y y} \sigma_{z z}-\sigma_{y z}^{2}\right]^{1 / 2}$ regardless of the shear value. The value $D_{\mathrm{S}}$ looses importance for $A(t)$ when shear increases (Dürbeck and Gerz, 1996). The same would be true for $D_{\mathrm{H}}$ if it were shear-independent.

\subsection{The turbulence model}

Most of the contrails form in the upper troposphere or lower stratosphere where strong winds, shear and stratification 
prevail (Birner et al., 2002; Houchi et al., 2010; Manney et al., 2011). Shear production of turbulence is driven by total shear $S_{T}$, while plume distortion follows the vertical shear $S$ of the plume-normal velocity,

$S_{T}^{2}=(d U / d z)^{2}+(d V / d z)^{2}, S=d V_{n} / d z$.

The Brunt-Vaisaila frequency $N_{\mathrm{BV}}$ measures stratification, and the Richardson number $R i$ the ratio of both.

$N_{\mathrm{BV}}^{2}=(d \Theta / d z) g / \Theta, R i=N_{\mathrm{BV}}^{2} / S_{T}^{2}$.

Molecular diffusion is negligible at plume scales because of large Reynolds numbers. The state of turbulence in this region is highly anisotropic and often composed of largescale horizontal quasi $2 \mathrm{~d}$ motions with little vertical motions. These motions are composed of wavy motions and intermittent turbulent spots (Dewan, 1979; Dörnbrack and Dürbeck, 1998; Riley and Lindborg, 2008). The kinetic energy spectrum follows a -3 -power law for the large scales and a $-5 / 3$-power law for smaller scales, suggesting either $2 \mathrm{~d}$ turbulence or $3 \mathrm{~d}$ inertial range turbulence, but local isotropy requires smaller scales (Nastrom and Gage, 1985; Riley and Lindborg, 2008). Wavy motions advect and distort the plumes without mixing. As mentioned before, the dissipation rate is often small (Schumann et al., 1995; Kantha and Hocking, 2011; Gultepe and Starr, 1995; Clayson and Kantha, 2008). Vertical motions in stratified air are limited by kinetic energy convertible to potential energy (buoyancy scale $\left.L_{B}=w^{\prime} / N_{\mathrm{BV}}\right)$. Overturning turbulence occurs only for scales below the Ozmidov scale $L_{\mathrm{Oz}}=\epsilon^{1 / 2} N_{\mathrm{BV}}^{-3 / 2}$ (Riley and Lindborg, 2008). Only dissipating turbulence causes essential mixing between plume air and ambient air. The horizontal scales $B$ of aged contrails are often in the transition region between the $2 \mathrm{~d}$-turbulence controlled by shear and stratification and even the smaller vertical scale $D$ is often far too large for being within the isotropic inertial range turbulence. Estimates of kinetic energy from subgrid scale models require knowledge of the Richardson number and the length scales of mixing and dissipation (Schumann, 1991), see Appendix A9, which is uncertain for NWP grids with grid scales far outside the inertial range. Turbulence experienced by cruising aircraft (including but not restricted to clear air turbulence) can be estimated from coarse NWP fields (Sharman et al., 2005; Frehlich and Sharman, 2010) but the scaling of this turbulence (composed of waves and turbulence) to plume scales has still to be investigated.

Since the plume trajectory meanders with ambient air motions at the scales of the contrail segments by advection in a Lagrangian manner, internal mixing is limited to motions at segment scales. The assumption of the same constant diffusivities for young and aged contrails would certainly not be appropriate. Instead, the diffusivities depend on shear and stratification at plume scales. The diffusivity model used here is based on classical fluid dynamics arguments, such as
Prandtl's mixing length for shear driven mixing and equilibrium between dissipation and energy production by shear and (negative) buoyancy, due to vertical mixing in stably stratified fluids (Hunt et al., 1988; Schumann, 1991; Schumann and Gerz, 1995).

Vertical diffusivities derived from measurements in the free atmosphere are typically $(0-1) \mathrm{m}^{2} \mathrm{~s}^{-1}$ (Pavelin et al., 2002). Only slightly larger values occur locally in buoyant plumes (Pisso et al., 2009) or in breaking gravity waves (Dörnbrack and Dürbeck, 1998). Smaller values, (00.6) $\mathrm{m}^{2} \mathrm{~s}^{-1}$, should be effective at plume scales. The horizontal diffusivities within a Lagrangian plume are of order (5-20) $\mathrm{m}^{2} \mathrm{~s}^{-1}$ for young plumes but grow to the order of $10^{4} \mathrm{~m}^{2} \mathrm{~s}^{-1}$ for wider and thicker plumes (Schumann et al., 1995; Dürbeck and Gerz, 1996; Pisso et al., 2009). Large eddy simulations are prone to numerical diffusion in particular in the presence of shear and stratification (Dürbeck and Gerz, 1995, 1996). They compute mixing in an Eulerian frame and do not identify the Lagrangian and scaledependent mixing. Therefore, differences between analytical plume models and large eddy simulations (Naiman et al., 2010; Unterstrasser and Gierens, 2010a) can hardly be used to constrain the model parameters for diffusivities.

Vertical mixing can be parameterized either for given dissipation rate $\epsilon$ or for given root-mean-square (rms) vertical turbulence fluctuations $w_{N}^{\prime}$ (Schumann and Gerz, 1995). Here, we prescribe $w_{N}^{\prime}=0.1 \mathrm{~m} \mathrm{~s}^{-1}$ to compute

$D_{\mathrm{V}}=\frac{c_{\mathrm{V}}}{N_{\mathrm{BV}}} w_{N}^{\prime 2}+f_{T} V_{T} D_{\mathrm{eff}}, c_{\mathrm{V}}=0.2, f_{T}=0.1$,

$D_{\mathrm{H}}=c_{\mathrm{H}} D^{2} S_{T}, D_{\mathrm{S}}=0, c_{\mathrm{H}}=0.1$

(To avoid division by zero, we constrain $N_{\mathrm{BV}}>0.001 \mathrm{~s}^{-1}$.) The value of $w_{N}^{\prime}$ is related to the dissipation rate by

$\epsilon=A_{\mathrm{S}} w_{N}^{\prime 2} S^{2}, A_{\mathrm{S}}=0.5$

(Hunt et al., 1988; Schumann and Gerz, 1995). Typical shear values, $S=\left(10^{-3}-10^{-2}\right) \mathrm{s}^{-1}$, and typical stratifications, $N_{\mathrm{BV}}=(0.01-0.03) \mathrm{s}^{-1}$, for $D=100 \mathrm{~m}$, imply $D_{\mathrm{V}} \approx(0.07-$ $0.2) \mathrm{m}^{2} \mathrm{~s}^{-1}, \quad D_{\mathrm{H}} \approx(1-10) \mathrm{m}^{2} \mathrm{~s}^{-1}, \quad$ and $\epsilon \approx\left(5 \times 10^{-7}-\right.$ $0.05) \mathrm{m}^{2} \mathrm{~s}^{-3}$. These values are within the range of measured data (Schumann et al., 1995). The buoyancy and Ozmidov scales are astonishingly small in this range, $L_{B} \approx(3-10) \mathrm{m}$, $L_{\mathrm{Oz}} \approx(0.07-0.7) \mathrm{m}$, i.e. not very much larger than the Kolmogorov scale $L_{\mathrm{K}}=\left(v^{3} / \epsilon\right)^{1 / 4} \approx(0.3-6) \mathrm{mm}$ where kinematic viscosity $v$ becomes important.

For quasi-steady homogeneous turbulence at low Richardson numbers, $c_{\mathrm{V}}$ is a constant and $c_{\mathrm{V}}=0.2$ is supported by atmospheric boundary layer measurements (Hunt, 1985). For large values of $R i, c_{\mathrm{V}}$ decreases (Schumann and Gerz, 1995) but $N_{\mathrm{BV}}$ gets large and, hence, $D_{\mathrm{V}}$ gets small in this limit anyway, so that this decrease is less important. $D_{\mathrm{H}}$ would become unrealistically large when replacing the mixing length 
scale $D$ in Eq. (36) with the dimensionally possible alternative $B$. The coefficient $c_{\mathrm{H}}=0.1$ is a free adjustable model coefficient. Its value is of minor importance because mixing is mainly controlled by the product of $D_{\mathrm{V}} S$ and less by $D_{\mathrm{H}}$, see Eq. (32). The essential free parameter is $w_{N}^{\prime}$ which measures the turbulence level at flight altitude.

Ice particles in contrails differ from passive tracers in that they sediment, grow or sublimate, release latent heat, and interact with radiation. Larger ice particles efficiently increase the effective depth of a contrail by sedimentation (Schumann, 1994; Atlas et al., 2006). We account for the vertical growth of the contrail cross-section by increasing the vertical diffusivity for given fall velocity $V_{T}$ (as computed below). Also $f_{T}$ is an important adjustable parameter.

Except for the constraint $D_{\mathrm{S}}^{2} \leq D_{\mathrm{V}} D_{\mathrm{H}}$, little is known about the off-diagonal "shear" diffusivity $D_{\mathrm{S}}$ (which has either sign). The shear diffusivity increases $A$ only if $S D_{\mathrm{S}}<0$, see Eqs. (32) and (31). This sign is imposed by shear driven correlations between vertical and horizontal velocity fluctuations.

$D_{\mathrm{S}}=-C_{\mathrm{DS}}\left(D_{\mathrm{V}} D_{\mathrm{H}}\right)^{1 / 2} S /|S|$.

As noted before, the value of $D_{\mathrm{S}}$ is unimportant for strong shear. Therefore, in the absence of further information, we set $D_{\mathrm{S}}=0$.

The shear $S$ is determined numerically from the wind field provided by the NWP model. The vertical grid spacing $\Delta z$ in such models is usually far larger than the contrail depth $D$. Hence, the magnitude of the shear at contrail scale may be larger because of shear by subgrid velocity fluctuations. The effective vertical resolution $\Delta z_{\text {eff }}$ in respect to shear is usually larger than the numerical resolution $\Delta z$. By comparisons to radiosondes, Houchi et al. (2010) show that the effective vertical resolution of the ECMWF model for shear is about $\Delta z_{\text {eff }}=2000 \mathrm{~m}$. We, therefore, use a shear value which is enhanced by a factor

$f_{\mathrm{S}}=\left(1+\left(\Delta z_{\mathrm{eff}} / D\right)^{n}\right) / 2, n \geq 0$.

This can be justified by considering the relationship between the mean squared shear and the kinetic energy spectrum $E(k)$ of homogeneous turbulence versus wave number $k$ (Lilly, 1967):

$\left\langle\left(\frac{\partial u}{\partial z}\right)^{2}\right\rangle \simeq \int_{0}^{\pi / \Delta z} k^{2} E(k) d k$.

The angular brackets denote a suitable statistical mean operator. For $E(k) \sim k^{-m}$, follows

$\left\langle\left(\frac{\partial u}{\partial z}\right)^{2}\right\rangle \sim(D / \Delta z)^{3-m}$.

For isotropic turbulence in Komogorov's inertial subrange, $m=5 / 3$, and hence $n=2 / 3$. For stably stratified flows, one expects steeper spectra, e.g. $m=3$, and hence $n=0$. The true value of $n$ needs to be determined empirically (Adelfang, 1971; Riley and Lindborg, 2008). For this model, we assume $n=1 / 2$.

The upper panels of Fig. 7 illustrate the order of magnitude of the contrail mixing properties computed this way. The vertical and horizontal diffusivities, $D_{\mathrm{V}}$ and $D_{\mathrm{H}}$, are small initially and grow with time when the contrails spread. $D_{\mathrm{H}}$ is about 10-100 times larger than $D_{\mathrm{V}}$. The details depend on age, shear, and stratification and, for high supersaturation, also on sedimentation. The diffusivity values for the early period, $(0.01-0.5) \mathrm{m}^{2} \mathrm{~s}^{-1}$ vertically and $(5-20) \mathrm{m}^{2} \mathrm{~s}^{-1}$ horizontally, are fully consistent with estimates derived from measurements and large eddy simulations (Dürbeck and Gerz, 1996; Schumann et al., 1995). Also the later values are consistent with observations (Pisso et al., 2009). The thickness and width values, $D$ and $B$, are of the right order of magnitude compared to ground-based lidar and satellite observations (Detwiler and Pratt, 1984; Freudenthaler et al., 1995; Duda et al., 2004; Atlas et al., 2006; Immler et al., 2008; Iwabuchi et al., 2012). More detailed comparisons have still to be made.

\subsection{Contrail ice mass integration in time}

The ice content changes with time according to mixing of the water mass mixing ratio in the plume with humidity from the ambient air. To simulate this process, we consider the mass budgets of contrail air mass $M=\rho A L$ and contrail water mass $M_{\mathrm{H}_{2} \mathrm{O}}=M(I+q)$ per contrail segment. The humidity $q$ inside the plume is assumed, as before, at ice-saturation, $q=q_{\mathrm{s}}$.

The air mass $M\left(t_{+}\right)$at $t_{+} \equiv t+\Delta t$ is

$M\left(t_{+}\right)=\rho\left(t_{+}\right) A\left(t_{+}\right) L\left(t_{+}\right)$.

Here, $\rho\left(t_{+}\right)$is computed for given ambient pressure and temperature at $t_{+}$, and the contrail cross-section $A\left(t_{+}\right)$from Eq. (3). The segment length $L$ is computed for the waypoint geometry.

The water mass $M_{\mathrm{H}_{2} \mathrm{O}}$ in the segment is composed of contrail water in the ice phase and water in the vapor phase at ice-saturation. The water mass budget changes by mixing with humid ambient air. The ambient air contains water vapor at mass specific concentration $q_{\mathrm{a}}$, and it may be supersaturated or subsaturated. Hence, at the end of the time step, the amount of water mass in the contrail is

$M\left(t_{+}\right)\left(I\left(t_{+}\right)+q_{\mathrm{s}}\left(t_{+}\right)\right)=M(t)\left(I(t)+q_{\mathrm{s}}(t)\right)+\Delta M q_{\mathrm{a}}$,

with contrail mass change $\Delta M=M\left(t_{+}\right)-M(t)$, and ambient humidity $q_{\mathrm{a}}=\left(q_{\mathrm{a}}\left(t_{+}\right)+q_{\mathrm{a}}(t)\right) / 2$ on average during the time step. We do not include ice from ambient cirrus and ignore the latent heat release for phase changes, because the resultant temperature changes are small, see Appendix A6. As a consequence, we find

$I\left(t_{+}\right)=\left[M(t)\left(I(t)+q_{\mathrm{s}}(t)\right)+\Delta M q_{\mathrm{a}}\right] / M\left(t_{+}\right)-q_{\mathrm{s}}\left(t_{+}\right)$. 

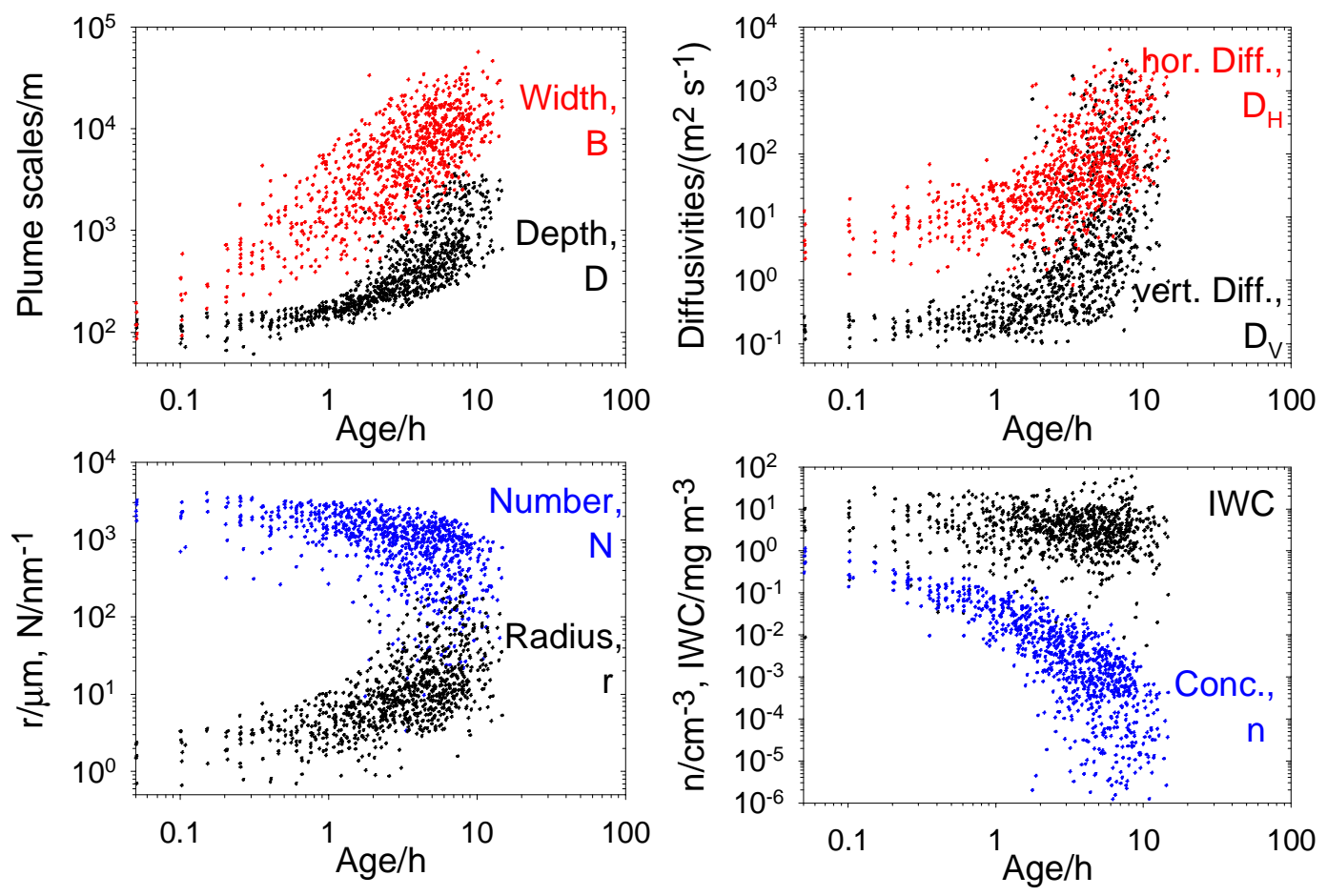

Fig. 7. Contrail properties versus contrail age in a set of contrail segments in the North Atlantic region during 6-9 June 2006. Top-left panel: Width $B$ and depth $D$. Top-right: Horizontal and vertical diffusivities $D_{\mathrm{H}}$ and $D_{\mathrm{V}}$. Bottom-left: Total number of ice particles per nanometer flight distance $N$, ice particle volume mean radius $r$, Bottom-right: Ice particle number concentration per volume $n$, and ice water mass content per volume $\rho I$.

Equations $(42,44)$ are used in CoCiP in their discrete form to compute $M\left(t_{+}\right)$and $I\left(t_{+}\right)$for each time step. In case of mixing with subsaturated ambient air and for decreasing saturation vapor inside the plume, the contrail may dry out. In this case, we set $I\left(t_{+}\right)=0$.

Here, we could distinguish contrails inside and outside the cloud covered part of a NWP grid cell, as assumed in the ECMWF model. Tompkins et al. (2007) assumed ice saturation inside the cloudy part of the cell, $q_{\mathrm{a}}=q_{\mathrm{s}}$. Outside the cloudy part, $q_{\mathrm{a}}=\left(q-C q_{\mathrm{s}}\right) /(1-C)$, where $C$ is the cloud cover in the grid cell. However, assuming that contrails occur uniformly inside the grid cell, with a share $C$ inside clouds and a share $1-C$ outside clouds, implies that the ambient humidity $q_{\mathrm{a}}$ equals on average the grid mean value, $q_{\mathrm{a}}=q$. Still, there could be differences in the contrail properties inside and outside of clouds because of nonlinearities. Full treatment of such differences would require considering two types of contrails, within and outside cloudy parts of a grid cell. In view of many other uncertainties in NWP humidity predictions, we ignore these differences in the present $\mathrm{CoCiP}$ version.

For interpretation, we note that Eqs. $(42,44)$ are discrete representations of

$d I / d t=(d M / d t)\left(q_{\mathrm{a}}-q_{\mathrm{s}}-I\right) / M-d q_{\mathrm{s}} / d t$.
We see that this differential equation describes the uptake of ice water mass from the ambient air in supersaturated air masses and the loss of ice water in subsaturated air masses according to the change in contrail mass $M(t)$. The ice water content of the contrail adapts to changes of saturation humidity inside the contrail with given plume mass. The contrail mass and the ice content grow mainly by mixing with ambient air masses. If the ambient air is supersaturated, this supersaturation of the air entrained into the plume is converted to ice. If the ambient air is subsaturated, ice particles in the plume sublimate keeping the mixed plume in ice saturation until all ice is consumed. For $q_{\mathrm{a}}=q_{\mathrm{s}}$, we have $d I / d t=-I(d M / d t) / M$, i.e. the ice mass content gets distributed over a growing plume mass with decreasing concentration. Moreover, the ice water content of the contrail adapts to changes of saturation humidity due to temperature or pressure changes inside the contrail with given plume mass. Finally, we note that $I=$ const in this approximation for constant ambient conditions, as it should.

The lower-right panel of Fig. 7 shows that the ice water content of the contrails, at a given time instant, varies little with their ages. In fact, the IWC is mainly a function of ambient ice supersaturation and temperature. Temperature determines the amount of water mass condensable for 
given relative humidity, consistent with empirical relationships (Schumann, 2002; Schiller et al., 2008), see Fig. 8.

\subsection{Contrail lifetime and ice number integration}

For given ice water content $I$ and total number of ice particles $N$, we compute the ice particle number concentration per volume $n$ and the volume-mean ice particle radius $r$ (needed as input for sedimentation and optical depth computations)

$n=N / A$,

$r=\left[\rho I /\left(n \rho_{\text {ice }} 4 \pi / 3\right)\right]^{1 / 3}$.

The volume mean radius is the radius of a volume-equivalent ice-particle sphere. It does not imply assuming spherical ice particles. Here, $\rho_{\text {ice }}=917 \mathrm{~kg} \mathrm{~m}^{-3}$ is a commonly used bulk density of ice particles. Mixing of air between the contrail plume and ambient air conserves $N$ but reduces $n$ by spreading the ice particles over an increasing plume cross-section.

Without microphysical loss processes (and without nucleation, without mixing with ambient cirrus, and for constant segment length $L$ ), the total number $N$ of ice particles remains conserved. (The change in segment length $L(t)$ is taken into account, see Eq. (56), later). As a consequence, contrails would stay infinitely for constant ice supersaturation, although getting thinner with time.

The lifetime of contrail clusters should be similar to the lifetime of ice supersaturated regions (ISSR) (Gierens et al., 1999). However, there are important differences: The lifetime of contrails is shorter because flight routes only exceptionally start when the air mass becomes supersaturated first. The lifetime of individual contrails may be shorter than the lifetime of contrail clusters. Moreover, the phase speed of contrails and ISSR regions is basically different. Contrails get advected with the wind while ISSR regions may be stagnant (at mountains) or move slower (in the upward motion branch of cyclones, i.e. in so-called conveyor belts).

The lifetime of ISSR has been estimated from observations to be of the order of hours (Immler et al., 2008). A mean flight path length in ISSR of $150 \mathrm{~km}$, with a standard deviation of $250 \mathrm{~km}$, was derived from humidity measurements onboard commercial aircraft (Gierens and Spichtinger, 2000); hence, there were many small ISSR regions. The longest distance flown inside an ISSR extended over more than $3700 \mathrm{~km}$ (Gierens and Spichtinger, 2000). The mean values are consistent with observed contrail cluster diameters of a few $100 \mathrm{~km}$ (Mannstein et al., 1999). If age scales with this length and with the mean wind speed (possibly of order $20 \mathrm{~m} \mathrm{~s}^{-1}$ ), a maximum ISSR age of about $1 \mathrm{~d}$ is to be expected, but most have likely shorter lifetimes. In two case-studies, at different scales, the lifetime derived from ECMWF trajectories was 6 and $24 \mathrm{~h}$ (Spichtinger et al., 2005b,a). Values exceeding $1 \mathrm{~d}$ are consistent with contrail cluster observations (Bakan et al., 1994).
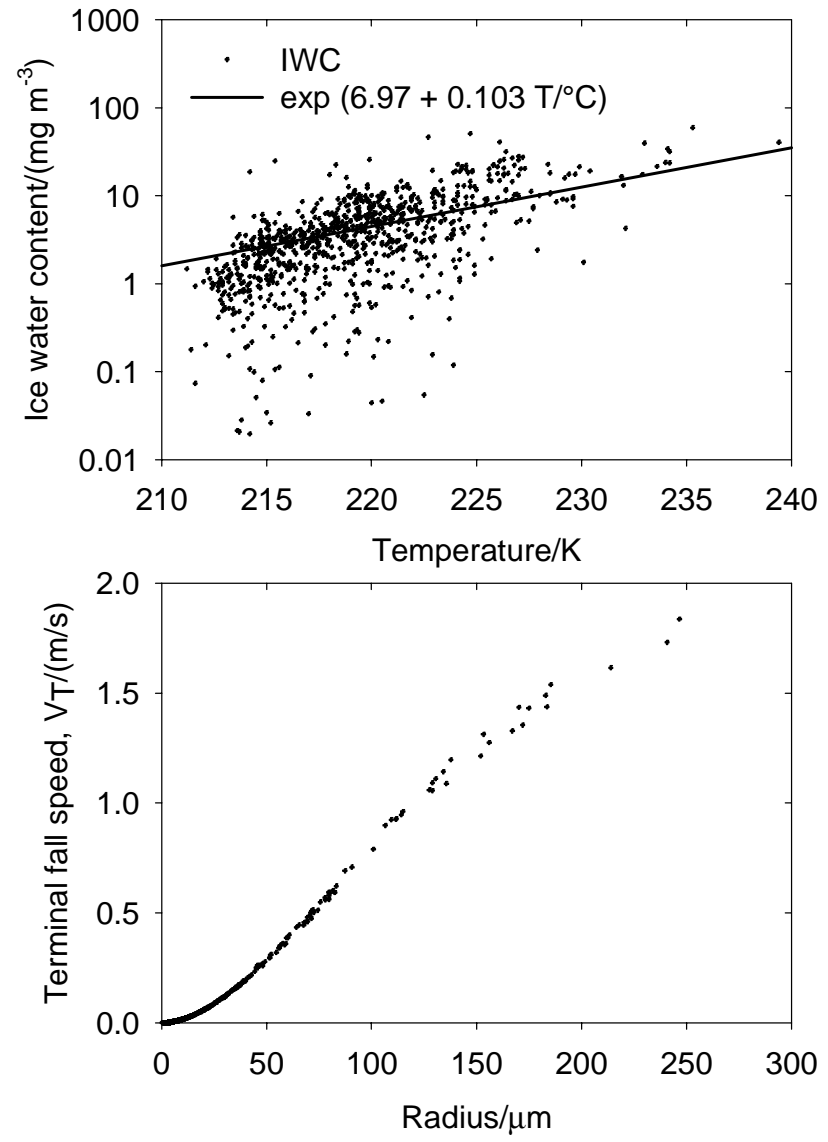

Fig. 8. Top: Ice water content versus temperature in a set of contrail segments as in Fig. 7, compared to an empirical approximation (Schumann, 2002). Bottom: Terminal fall velocities versus particle radius Ice water content versus temperature in the same set of contrail segments.

Here, we estimate an aviation-related lifetime of ISSR regions by computing the age of trajectories which start at aicraft flight waypoints satisfying the SAC in ice supersaturated air and last until the ambient humidity drops below ice saturation. For this purpose, we use the advection scheme for a passive tracer (without sedimentation) with ECMWF data for 6-9 June 2006. Most of such trajectories end after less than one hour, see Fig. 9, the mean, median, and maximum (mean, med, max) ages of these aircraft-related ISSR trajectories are computed this way as $9.5 \mathrm{~h}, 7.3 \mathrm{~h}$, and $>36 \mathrm{~h}$, respectively. Apparently, the age frequency distribution follows an exponential function. Based on such a fit, the mean and median ages are 14.6 and $10.1 \mathrm{~h}$. Simulated ISSR lifetimes exceeding $24 \mathrm{~h}$ are found for about $1 \%$ of all flights, which is not unrealistic (Bakan et al., 1994).

When we apply CoCiP without particle number loss processes, we compute even larger ages, see Fig. 9. The mean, med, max ages of these contrails would be $11.2 \mathrm{~h}, 8.7 \mathrm{~h}$, $>36 \mathrm{~h}$. The larger lifetime can be explained by the reservoir 


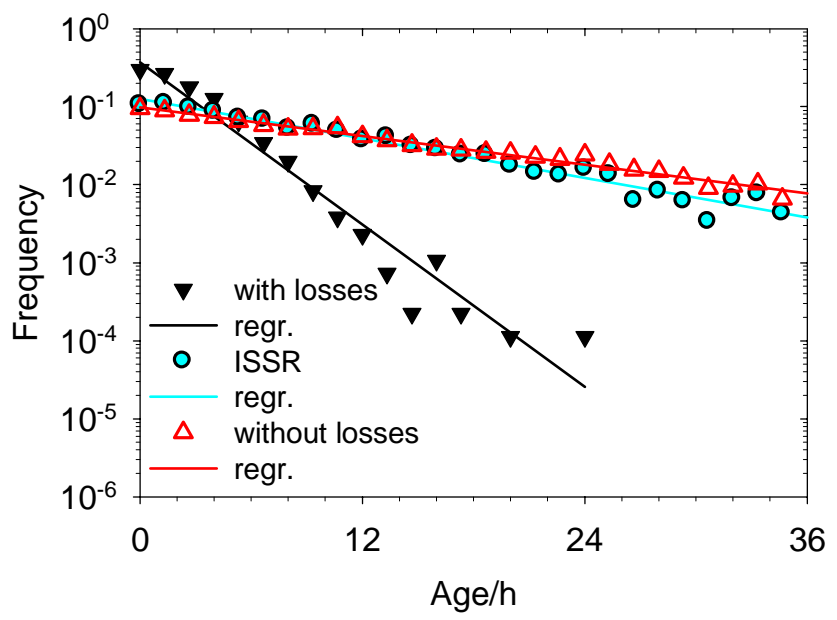

Fig. 9. Age frequency of ice supersaturated regions (cyan: ISSR) and of contrails (black: with losses, red: without losses) in a set of contrail segments as in Fig. 7

of ice water built up while staying in the ISSR, which is maximum just when the ISSR regime ends, and which takes time to sublimate. At this time $t$, the contrails have large cross-sections $A(t)$. It takes a further time of order $\tau_{\text {dil }}=$ $A /(d A / d t)$ to dilute the plume to subsaturation. For dilution following a power-law with time, $A(t)=A_{0} t^{n}$, and for $n \approx 0.8$ (Schumann et al., 1998), the mixing time $\tau_{\mathrm{dil}}=t / n$ is large and the total contrail age $t+\tau_{\mathrm{dil}}$ could reach twice the age of ISSR masses.

The lifetime of contrails is highly variable (Detwiler and Pratt, 1984). Contrail ages up to $18 \mathrm{~h}$ and possibly more have been observed (Bakan et al., 1994; Minnis et al., 1998; Haywood et al., 2009). These ages may be exceptions, not the rule. Contrails were traced in time with data from geostationary (Duda et al., 2001, 2004; Vazquez-Navarro, 2009) and polar orbiting satellites (Atlas et al., 2006). Contrails become first visible in these data after a dwell time of about $(0.5-1) \mathrm{h}$, depending on satellite resolution (Atlas and Wang, 2010). Contrail clusters were often followed for (2-5) h (Duda et al., 2001, 2004). An automatic contrail-tracking algorithm identified many contrails in METEOSAT satellite scenes with mean, med, max ages (without this dwell time) of $51 \mathrm{~min}$, $20 \mathrm{~min}, 14 \mathrm{~h}$, respectively (Vazquez-Navarro, 2009). Hence, we expect median contrail lifetimes of order of a few hours, definitely shorter than what is computed without losses.

Therefore, we add particle loss models to CoCiP. The model contains three of them:

$$
(d N / d t)_{\mathrm{loss}}=(d N / d t)_{\mathrm{turb}}+(d N / d t)_{\mathrm{agg}}+(d N / d t)_{\mathrm{meso}}
$$

Unfortunatley, we found very little theory in the literature which would allow to parameterize contrail or cirrus ice particle loss processes within the framework of a bulk model. The models used are mainly based on dimensional analysis. The modeled loss processes are designed to simulate (turb:) particle losses by plume-internal turbulence, (agg:) sedimentation-induced aggregation (Sölch and Kärcher, 2010), and (meso:) losses induced by turbulent humidity fluctuations by mesoscale turbulence and gravity waves (Kärcher and Ström, 2003) and possibly plume turbulence (Gierens and Bretl, 2009; Unterstrasser and Gierens, 2010a). Also radiative heating of the contrail plume and of individual (large) ice particles may contribute to particle losses (Gierens, 1994; Chlond, 1998; Jensen et al., 1998a; Dobbie and Jonas, 2001; Unterstrasser and Gierens, 2010b). Efficient treatment of radiative heating is possible (similar to radiative forcing, see Sect. 2.14), but not considered in this paper (Schumann et al., 2010).

Mixing of contrails with dry ambient air masses was considered for ice mass in the Sect. 2.11. If the ice size distribution would be narrow and if the plume is well mixed, then it seems conceivable that the number of ice particles stays constant until all ice mass is sublimated, when all particles disappear suddenly. However, this is not realistic (Gierens and Bretl, 2009). While growth of particles would narrow the size distribution, turbulent mixing of cloud air masses with particles of different sizes tends to broaden the size distribution. Eventually, mixing causes some of the smaller particles to sublimate. We roughly model this with the first loss term, "turb",

$(d N / d t)_{\text {turb }}=-E_{T}\left(\frac{D_{\mathrm{H}}}{\max (B, D)^{2}}+\frac{D_{\mathrm{V}}}{D_{\text {eff }}^{2}}\right) N$

with $E_{T}=1$ as an adjustable parameter. The maximum function avoids large losses for the initially narrow plume, when $B<D$, because the initial losses are included in the survival factor, Eq. (22). The scale $D_{\text {eff }}$ is used instead of $D$ because it enhances the loss for vertically thin contrails. Parameter studies, with and without this and the other loss models, show that the "turb" part contributes to losses relatively to all loss terms most strongly at low ice supersaturation. Further improvements appear possible in future model versions (e.g. the losses may be humidity dependent).

Sedimentation limits the lifetime of contrails for high ambient supersaturation when the ice particles grow large (Schumann, 1996; Unterstrasser and Gierens, 2010a). During sedimentation, large falling particles may collide and aggregate with smaller ice particles so that the total number of ice particles $N$ decreases. In a size-resolved model, with $n_{i}$ representing the number density of ice particles in a size range $r_{i}-r_{i+1}$, the aggregation process could be parameterized by a rate equation (e.g. Pruppacher and Klett, 1997; Sölch and Kärcher, 2010)

$\left(d n_{k} / d t\right)_{\mathrm{agg}}=\frac{1}{2} \sum_{i+j=k} K_{i j} n_{i} n_{j}-n_{k} \sum_{j=1}^{\infty} K_{k j} n_{j}$,

with rate coefficients

$K_{i j}=E_{A} \pi\left(r_{i}+r_{j}\right)^{2}\left|V_{i}-V_{j}\right|$, 
accounting for aggregation due to collision of particles of different sizes $r_{i}$ and different fall speeds $V_{i}$. Since we have no explicit information on the particle size spectrum, we assume that the size spectrum in the contrails has a width of order $r$, so that $\left(r_{i}+r_{j}\right)^{2} \approx 4 r^{2}$, and $\left|V_{i}-V_{j}\right| \approx V_{T}(2 r) \approx 2 V_{T}(r)$. Hence, an approximate aggregation rate as a function of mean particle size $r$ is

$(d N / d t)_{\mathrm{agg}}=-E_{A} 8 \pi r^{2} V_{T} N^{2} / A$,

with an adjustable model parameter $E_{A}=1$. The terminal fall velocity $V_{T}(r, T, p)$ is computed as in Sölch and Kärcher (2010). The aggregation efficiency $E_{A}$ may depend on temperature (Sölch and Kärcher, 2010). However, the aggregation is unimportant anyway at low temperatures (Jensen et al., 2011). We find important contributions by aggregation only for temperatures larger about $220 \mathrm{~K}$ when the ice water content gets large (see Fig. 8).

Finally, we roughly parameterize the effect of subgrid scale vertical velocity fluctuations $w_{\text {meso }}^{\prime}$, e.g. from mesoscale turbulence and gravity waves (Kärcher and Ström, 2003). The velocity fluctuations induce temperature fluctuations depending on vertical stratification and mixing, $T_{\text {meso }}^{\prime} \approx$ $w_{\text {meso }}^{\prime} d T / d z$. Particles are assumed to get lost when $T_{\text {meso }}^{\prime}$ becomes large. In place for a better scale still to be found, we compare to the temperature change for doubling ice mass (Pruppacher and Klett, 1997),

$\Delta T_{c}=\left(R_{1} T^{2} / L_{\mathrm{s}}\right)$.

Here, $L_{\mathrm{S}} \approx 2.8 \times 10^{6} \mathrm{~J} \mathrm{~kg}^{-1}$ is the latent heat of sublimation and $R_{1}=461.5 \mathrm{~J}\left(\mathrm{~K} \mathrm{~kg}^{-1}\right.$ the gas constant for water vapor; typically, $\Delta T_{c} \approx 9 \mathrm{~K}$. Note that $w_{\text {meso }}^{\prime}$ is different from $w_{N}^{\prime}$, because it includes wave motions. Moreover, $w_{\text {meso }}^{\prime}$ is a mesoscale property related to a NWP grid scale $\ell$ and possibly plume segment length, but different from the plume cross-section scales. Hence, a separate turbulence model is needed for this. We estimate

$w_{\text {meso }}^{\prime 2}=w_{\text {SGS }}^{\prime 2}+\bar{w}^{2}$

for the variance $w_{\text {SGS }}^{\prime 2}$ of vertical SGS turbulence and of vertical motions $\bar{w}$ (possibly gravity wave driven) at NWP scales. The method to analyse SGS velocity variance follows Schumann (1991), his Eqs. (10, 12, 14). Details are given in Appendix A9. These velocity fluctuations $w_{\text {meso }}^{\prime}$ are mostly small, of the order (1-10) $\mathrm{cm} \mathrm{s}^{-1}$. We further assume that the time scale for reducing the number of ice particles by wave induced vertical fluctuations is of order $N_{\mathrm{BV}}^{-1}$. With these relations we set

$(d N / d t)_{\text {meso }}=-E_{\text {meso }} N_{\mathrm{BV}} w_{\text {meso }}^{\prime}(d T / d z) / \Delta T_{c}$,

Based on preliminary tests and comparisons of computed and observed contrail cirrus cover, we presently use this model with $E_{\text {meso }}=2$. Further studies are needed to test and possibly improve this model.
For keeping $N \geq 0$ during numerical integration of $N$ in time, even for strong loss processes, instead of using the standard Runge-Kutta scheme, we use an analytical integral (see Appendix A10), which provides the exact solution for constant coefficients and positive approximate solutions otherwise.

$N(t+\Delta t)=\frac{N(t) \beta \exp (-\beta \Delta t)}{\beta+\alpha y_{0}[1-\exp (-\beta \Delta t)]} \frac{L(t)}{L(t+\Delta t)}$.

(The length scale ratio corrects for the change in segment length during the time step.) The coefficients $\alpha$ and $\beta$

$\alpha=-\frac{\Delta t}{N^{2}}(\partial N / \partial t)_{\text {agg }}, \beta=-\frac{\Delta t}{N}(\partial N / \partial t)_{\text {turb }}$,

are computed as average of the values at times $t$ and $t+\Delta t$.

Figure 9 shows that the contrail ages get considerably reduced with these particle loss parameterizations. The contrail ages are now below the ISSR ages. The mean, med, max ages for this June-case are $2.9 \mathrm{~h}, 2.0 \mathrm{~h}, 24 \mathrm{~h}$. These ages are consistent with the observations cited. From parameter studies, we find that all three loss models are important for the mean statistics.

The computed particle concentrations are consistent with observations: The lower-left panel in Fig. 7 shows that the total ice crystal number $N$ in the ensemble of contrails ranges within $(1-50) \times 10^{11} \mathrm{~m}^{-1}$, slowly decreasing with plume age. For comparison, Spinhirne et al. (1998) deduced values of (1-3) $\times 10^{11} \mathrm{~m}^{-1}$ slightly decreasing with plume age from remote sensing of two contrails with estimated ages in the order of one or a few hours. The computations show ice particle concentrations $n$ decreasing more strongly than $N$ because of dilution from about $0.1 \mathrm{~cm}^{-3}$ to $0.001 \mathrm{~cm}^{-3}$ for 1-h aged contrails, which is roughly consistent with extensive in-situ observations in cirrus (Krämer et al., 2009). For aged contrails, one expects ice particle concentrations similar to ambient cirrus (Gayet et al., 1996; Spinhirne et al., 1998; Schröder et al., 2000; Schumann et al., 2011b).

The lower panel of Fig. 8 shows the relationship between particle sizes and fall speeds. The fall speeds depend on Reynolds number and, hence, the scatter of data reflects variations of viscosity with pressure and temperature. The range of computed sedimentation speeds is consistent with the few observations: Duda et al. (2004) found best agreement with observations for computed contrail positions, depending on wind shear and altitude, for fall speeds up to $0.045 \mathrm{~m} \mathrm{~s}^{-1}$. Konrad and Howard (1974) deduced fall speeds up to $1.4 \mathrm{~m} \mathrm{~s}^{-1}$ from radar observations in contrail fallstreaks.

\subsection{Contrail optical depth for solar radiation}

The radiative effect of a contrail segment is controlled by the product of the effective width $B$ of the contrail and its optical depth $\tau$ for solar radiation (at $550 \mathrm{~nm}$ ) from zenith. These 


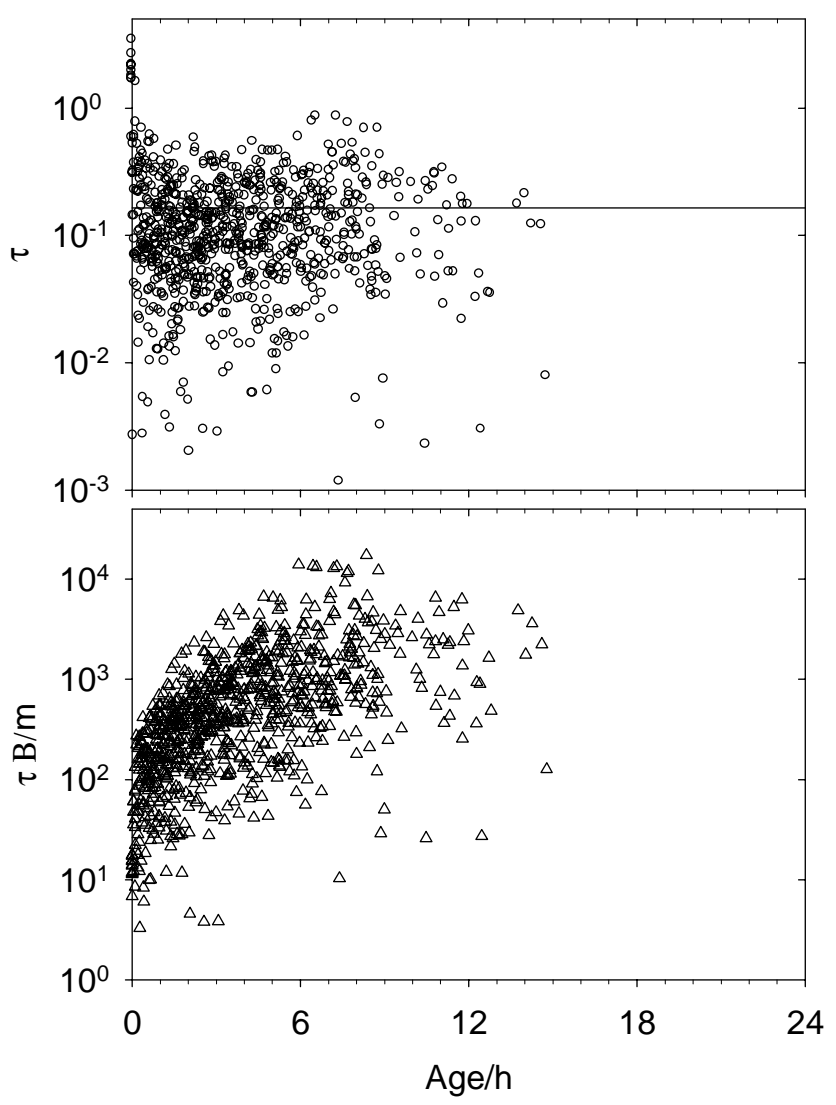

Fig. 10. Top: Optical depth $\tau$ at $550 \mathrm{~nm}$ versus contrail age in a set of contrail segments (as in Fig. 7); the line depicts the mean value (0.164). Bottom: Product $\tau B$ of optical depth times contrail width $B$ versus contrail age.

values follow from the horizontal and vertical integrals over the width and height of the contrail,

$B \tau=\beta \iint \exp \left[-\frac{1}{2} \mathbf{x}^{T} \sigma^{-1} \mathbf{x}\right] d z d y=\beta A$.

The extinction $\beta$ and the optical depth $\tau$ are related to the effective plume depth $D_{\text {eff }}$,

$\beta=3 Q_{\text {ext }} \rho I /\left(4 \rho_{\text {ice }} r_{\text {eff }}\right), \tau=\beta D_{\text {eff }}$,

$D_{\text {eff }}=A / B$.

The radiation extinction efficiency $Q_{\text {ext }}$ is computed using approximate Mie-theory

$Q_{\mathrm{ext}}=2-\left(4 / \rho_{\lambda}\right)\left(\sin \left(\rho_{\lambda}\right)-\left(1-\cos \left(\rho_{\lambda}\right)\right) / \rho_{\lambda}\right.$,

with $\rho_{\lambda}=4 \pi r_{\text {eff }}(\kappa-1) / \lambda, \kappa=1.31$ as the real refractive index of ice, and $\lambda=550 \mathrm{~nm}$ the wavelength of visible light (van de Hulst, 1957).
The effective radius $r_{\text {eff }}$ is defined by the ratio of particle volume $\bar{V}_{p}$ to particle projected area $\bar{A}_{p}$ (Hansen and Travis, 1974),

$r_{\mathrm{eff}}=\frac{3 \bar{V}_{p}}{4 \bar{A}_{p}}=\frac{r}{C_{r}}$.

The coefficient $C_{r}$ measures the ratio between volume mean radius and the effective radius. Its value $C_{r}=0.9 \pm 0.3$ depends on the particle shape and size distribution of the ice particles, which are variable, so that this value is uncertain (Schumann et al., 2011b).

According to Appendix A11, the local value of $\tau(y)=$ $f_{\tau}(y) \tau$ for given $y$, as needed for analysis of contrail cover for given optical depth, is a factor

$f_{\tau}(y)=(4 / \pi)^{1 / 2} \exp \left[-(1 / 2) y^{2} / \sigma_{y y}\right]$

larger than the bulk value $\tau ; f_{\tau}(0)=1.13$.

The contrail simulation ends when $\tau$ gets small $\left(<10^{-4}\right)$, or when the number concentration $n$ of ice particles gets small $\left(<1 \mathrm{~L}^{-1}\right)$, or when the contrail center of gravity falls below the lower boundary of the computational domain (typically at $600 \mathrm{hPa}$ ).

Figure 10 shows that the model computes optical depth values within the range $0.001-0.5$ in most cases, with larger values for young contrails. This is fully consistent with observations and other models (Kärcher et al., 2009a; Atlas and Wang, 2010; Voigt et al., 2011). However, with respect to climate impact, it is the product of $\tau$ with the contrail width $B$ which counts. This value generally increases and takes a maximum after about a few hours in this model, consistent with observations (Atlas and Wang, 2010). Only a few of the older contrails reach larger values. It implies that the largest climate impact comes from contrails a few hours of age.

\subsection{Radiative forcing}

Contrails induce a change in net radiances at top of the atmosphere, which is known as radiative forcing (RF). RF is commonly used for assessing the climate impact of contrails (IPCC, 2007). For CoCiP it is essential to have a parameterization which allows for efficient computation of RF for each contrail segment. The standard approach using complex radiation transfer models would be far too time consuming. Therefore, a new parameterization has been developed (Schumann et al., 2009, 2012) which is briefly summarized here.

The RF by contrails is computed separately for the longwave (LW) and shortwave (SW) spectral ranges. For each range, the local RF is computed as a function of the optical depth $\tau$, effective radius $r_{\text {eff }}$, and temperature $T$ of the contrail, and optical depth $\tau_{c}$ of cirrus above the contrail. The parameterization uses NWP input for outgoing longwave radiation (OLR), reflected solar radiation (RSR), and solar direct radiation (SDR) (Blanco-Muriel et al., 2001) for the given 
atmosphere without contrails. Further input includes the solar constant $S_{0}$ which varies with the day of the year a few percent around the annual mean value of about $1365 \mathrm{~W} \mathrm{~m}^{-2}$ (Blanco-Muriel et al., 2001; Paltridge and Platt, 1976).

From these input values, the model (in module RADI) computes the LW and SW RF per contrail area using given analytical functions,

$$
\begin{aligned}
& \mathrm{RF}_{\mathrm{LW}}=\mathrm{RF}_{\mathrm{LW}}\left(O L R, T, \tau, r_{\mathrm{eff}}, \tau_{c}\right) \geq 0, \\
& \mathrm{RF}_{\mathrm{SW}}=\mathrm{RF}_{\mathrm{SW}}\left(S_{0}, A_{\mathrm{eff}}, \mu, \tau, r_{\mathrm{eff}}, \tau_{c}\right) \leq 0,
\end{aligned}
$$

with effective albedo $A_{\text {eff }}=\mathrm{RSR} / \mathrm{SDR}$, and cosine of solar zenith angle $\mu=\cos (\theta)=\mathrm{SDR} / S_{0} . \mathrm{RF}_{\mathrm{LW}}$ is constrained to positive values, so that contrails at low altitudes with high ambient temperatures have zero contribution. Likewise, $\mathrm{RF}_{\mathrm{SW}}$, is constrained to negative values. The model prescribes the contrail habit mixture as a function of radius $r$ (Schumann et al., 2011b). The parameterization with 5 fit parameters for LW and 10 for SW has been calibrated for an extensive set of atmospheres/surfaces against libRadtran forward calculations (Mayer and Kylling, 2005). The approximate results and the libRadtran results correlate better than $98 \%$. The RF parameterization is consistent with previous results (Meerkötter et al., 1999; Schumann et al., 2012). Preliminary RF results have been presented in Schumann et al. (2011a).

\subsection{Contrail cirrus cover}

The ensemble of contrails together with the natural (nonaviation) NWP-cirrus is considered as the "total cirrus" in this model. The additional cirrus induced by contrails is interpreted as "contrail cirrus". Contrail cirrus differs from young isolated contrails in shape. Single contrails widen by turbulent diffusion and shear, get distorted by variable winds, and disrupted by contrail free segments, but still remain identifiable as a near-linear sequence of contrail segments in clear air. Single contrails change their shape when overlapping with natural cirrus. A cluster of contrails with different histories overlap irregularly so that the linear structure gets lost. $\mathrm{CoCiP}$ in this version does not account for physical interactions between contrails among each other nor with cirrus. However, CoCiP does simulate irregularly structured contrail cirrus.

We define the regional cover $C$ of a cirrus as that fractional horizontal area in which the optical depth $\tau$ of the cirrus is larger than a critical value $\tau_{c}$. This seems plausible, because only cirrus with a minimum optical depth is visible. Moreover, the optical depth controls its radiative forcing and, hence, its climate impact. Details of detectability depend on underground properties, ambient atmosphere, illumination, etc. The threshold is observer and instrument dependent. Hence, cloud cover may be ill-defined. Here, we orient ourselves on satellite observations, where a value of $\tau_{\mathrm{c}} \approx 0.1$ may be realistic.
Overlapping contrails are treated according to their known position and optical depth. The coverage of overlapping contrails is far smaller than the summed coverage of individual contrails. In regions with many overlapping contrails, the contrail cirrus cover increases less than linear with air traffic and saturates at a maximum value (which may be $100 \%$ ). The cover gets even smaller when contrails overlap with natural cirrus. Hence, it is important to have a clear definition of cover $C$. Here, we define the cover of contrail-cirrus (CO) as the cover of total cirrus (COCI) minus the cover of natural cirrus $(\mathrm{CI})$,

$C_{\mathrm{CO}}=C_{\mathrm{COCI}}-C_{\mathrm{CI}}$,

based on their optical depth values, respectively, above a critical value,

$\tau_{\mathrm{COCI}}>\tau_{\mathrm{c}}$ and $\tau_{\mathrm{CI}}>\tau_{\mathrm{c}}$.

More complex criteria, e.g. the contrast in brightness temperature and reflectance of contrails and cirrus, computed similar to RF, Sect. 2.14, might be included in future versions.

Here, the cover is computed pixel-wise based on optical depth. The NWP grid is far too coarse to resolve the contributions from the relatively narrow contrails. Instead, we use a fine-resolution matrix of optical depth values ("cloud mask") $\tau_{i, j}$ in grid cells (pixels) with far higher resolution and independent of the NWP grid. For global analysis, the grid center points are located at $x_{i}=i \Delta x, y_{j}=j \Delta y, i=0,1, \ldots, N_{x T}$, $j=0,1, \ldots, N_{y T}$, with grid spacing $\Delta x=360 /\left(N_{x T}-1\right)$, $\Delta y=180 /\left(N_{y T}-1\right)$. We use $N_{x T}=5001, N_{y T}=3601$ in our global reference cases. The pixel area size is $\Delta x \times \Delta y$. In this implementation its value is $5.7 \times 5.6 \mathrm{~km}^{2}$ at $45^{\circ}$ latitude. Even this fine grid cannot resolve the very narrow young contrails, but these contribute little to the total cover. The resolution error caused by finite grid spacings depends on the ratio of contrail width relative to grid resolution and on the threshold ratio $\tau_{c} / \tau$. It decreases with increasing contrail width and decreasing threshold. The error gets small in particular for many randomly distributed and overlapping contrails. For example, for a region over the North Atlantic, for $\tau_{c}=0.1$, mean width $B=7 \mathrm{~km}$, and mean optical depth $\tau=0.12$, the contrail cover changes by $5 \%$ if the grid scale is reduced by a factor 10. This appears to be acceptable.

The contrail contributions to $\tau_{i, j}$ are computed within a module TAU2DS within the loop over all flights. The NWPcirrus contributions are added in module TAU2DX at the end. As explained in Appendix A12 and Fig. A7, a segment contributes to $\tau_{i, j}$ when the normal from $x_{i}, y_{j}$ onto the segment $\left(W_{n}, W_{n+1}\right)$ crosses the segment at a cross-point $x_{s}, y_{s}$ between its endpoints. The amount $\tau f_{\tau}(s)$, see Eq. (63), of contribution depends on the distance $s$ between $x_{s}, y_{s}$ and $x_{i}, y_{j}$. In order to add all $\tau$-contributions from all contrail segments, one has to test the distance to all points $(i, j)$ in the cloud mask. This time-consuming test can be reduced to 
pixels in a certain distance from the segment end-points, depending on the maximum product $B \tau$ of the contrail segments. By this strategy, the computing time increases about linearly with the total number of contrail segments from all flights.

For analysis of contributions of natural cirrus to $\tau_{i, j}$ in the upper troposphere and lower stratosphere (between $z_{\min }=6.0 \mathrm{~km}$ and $z_{\max }=16.1 \mathrm{~km}$ ), we first compute the optical depth $\tau_{C}=\int_{z_{\min }}^{z_{\max }} \beta_{C} d z$ of this cirrus for given cirrusextinction $\beta_{\mathrm{C}}=3 Q_{C \mathrm{ext}} \mathrm{IWC}_{\mathrm{C}} /\left(4 \rho_{\text {ice }} r_{\text {eff }}\right)$, and extinction efficiency $Q_{C \text { ext }}=2$. The effective radius is determined from the NWP data as a function of temperature and ice water content $\mathrm{IWC}_{\mathrm{C}}$ of the NWP-cirrus. When using ECMWF data, we compute $r_{\text {effC }}$ consistently (Sun, 2001; Sun and Rikus, 1999).

Moreover, we account for the $3 \mathrm{~d}$ distribution of cirrus cover $C_{\mathrm{C}}(x, y, z, t)$ inside a cirrus, which is provided as input for each NWP grid cell: For given $C_{\mathrm{C}}(x, y, z, t)$, we first compute a $2 \mathrm{~d}$ cirrus cover effective for observers from above, $C i_{\mathrm{C}}(x, y, t)=\max _{z}\left[C_{\mathrm{C}}(x, y, z, t)\right]$. Since the cloud mask has a far higher resolution than the NWP-grid, we distribute the broken cirrus coverage in the cloud mask randomly. For this purpose, we distribute the cirrus optical depth $\tau_{\mathrm{C}}$ in at least partially covered grid cells onto pixels, keeping its grid mean value constant at $\tau_{C}$. For each grid cell $i, j$ with $C i_{\mathrm{C}}>0$, a random number $R$, uniformly distributed within $0-1$, decides, if $R>1-C i_{\mathrm{C}}$ then $\tau_{c}=\tau_{\mathrm{C}} / C i_{\mathrm{C}}$, else $\tau_{c}=0$. The value $\tau_{c}$ is added to $\tau_{i, j}$ as cirrus contribution. The randomly distributed cloud cover is evident in Figs. 11-12.

For example, the top panel of Fig. 11 shows the optical depth of contrails $\left(\tau_{\mathrm{CO}}\right)$ for all the contrails identified by red lines in Fig. 6, while the lower panel shows the optical depth of contrails and cirrus together $\left(\tau_{\mathrm{COCI}}\right)$. Figure 11 (upper panel) exhibits again clusters of contrails over North America, the North Atlantic and mid Europe. Some contrails plotted in Fig. 6 (e.g. near $90^{\circ}$ latitude in the Southern Indian Ocean) are invisible in Fig. 11, because of low optical depth. When plotted together with the optical depth of natural cirrus (lower panel, only few contrails (e.g. over North America) remain visible. This plot shows the optical depth of ice clouds above $6 \mathrm{~km}$ altitude. This includes deep convective clouds with quite large optical depth, in particular in the tropics.

Figure 12 shows the computed cover for a contrail outbreak over parts of North America. The upper part shows the cover $C_{\mathrm{CO}}$ of contrails $\left(\tau_{\mathrm{CO}}>0.1\right)$ alone, while the lower panel shows the cover of total cirrus $C_{\mathrm{COCI}}$ as composed of natural cirrus $\left(\tau_{\mathrm{CI}}>0.1\right.$, blue pixels) and contrail-cirrus ( $\tau_{\mathrm{CI}}<0.1$ and $\tau_{\mathrm{COCI}}>0.1$, red pixels). Contrails inside (or above/below) thick cirrus do not contribute to contrail-cirrus cover. In fact, a large fraction of contrail pixels (about 2/3 globally) become invisible inside natural cirrus. However, even thin contrails may push the optical depth of thin cirrus above the critical value. The contrail outbreak happens to occur in this simulation over North America (at $10 \mathrm{pm}$ local time). Some contrails are still identifiable as linear structures.
However, the linear shape gets lost in contrail clusters, which mostly occur in the neighborhood of natural cirrus. Still part of the contrail pixels remain visible in the total cirrus results while others submerge in natural cirrus. Some of the additional contrail cirrus pixels are closing cloud gaps in the natural cirrus cover. Regionally, over (100-600) km scales in this example, the contrails cause $100 \%$ cirrus cover.

For this cloud mask, we finally compute global or regional cloud covers by summing the areas of pixel cells with $C_{\mathrm{COCI}}=1$ and $C_{\mathrm{CI}}=0$, and dividing by the respective global or regional total horizontal area. The results depend nonlinearly on the threshold value $\tau_{c}$. For $\tau_{c}=0.1$ and 0.05 , in this example, for a single time slice, the global contrail coverage is $0.23 \%$ and $0.36 \%$ (NWP cirrus cover: $19 \%$ and $27 \%$ ), respectively. Hence, the value of $\tau_{c}$ has to be carefully selected for meaningful comparisons to observations.

\section{Results and discussions}

\subsection{Example simulation of contrails}

As discussed before, see Figs. 6, 11, and 12, CoCiP has been applied for a global case study using NWP data from ECMWF and traffic data from FAA-EUROCONTROL. The global simulation identifies 31575 flights in the time period 03:00-06:00 UTC 6 June 2006, forming at least short contrails. In this simulation, the time step is $\Delta t=1 \mathrm{~h}$. The computation time is $3.5 \mathrm{~min}$ (including a large fraction for $\mathrm{I} / \mathrm{O}$ time). Hence, for each contrail, less than $0.01 \mathrm{~s}$ is needed. For a snapshot at 06:00 UTC that day, a total of 3402 contrails are found. This clearly demonstrates the size of the problem and the efficiency of the method. Still, to simulate a full year hourly with an order 30 million flights globally, requires about $7 \mathrm{~h}$ of computing time.

Figures 7-10 show the contrail properties for a random subset of contrail segments over the North Atlantic. As discussed above, the resultant contrail dimensions and ice properties exhibit magnitudes and trends with age at least roughly consistent with observations. The simulations were performed with model parameters as explained. The most important parameters and their values are set as listed in Table 2. For quantitative assessment, we next consider case studies for single contrails.

\subsection{Dilution}

The dilution ratio of passive tracers emitted form aircraft was discussed before. It determines the mass specific concentration above background for given emission index EI as $\Delta c=\mathrm{EI} / N_{\text {dil }}$. The empirical function, Eq. (16), was derived from measurements for plume ages $t$ up to $5000 \mathrm{~s}$, with about factor three uncertainty (Schumann et al., 1998). A rather large spread of the data relative to this approximation had to 

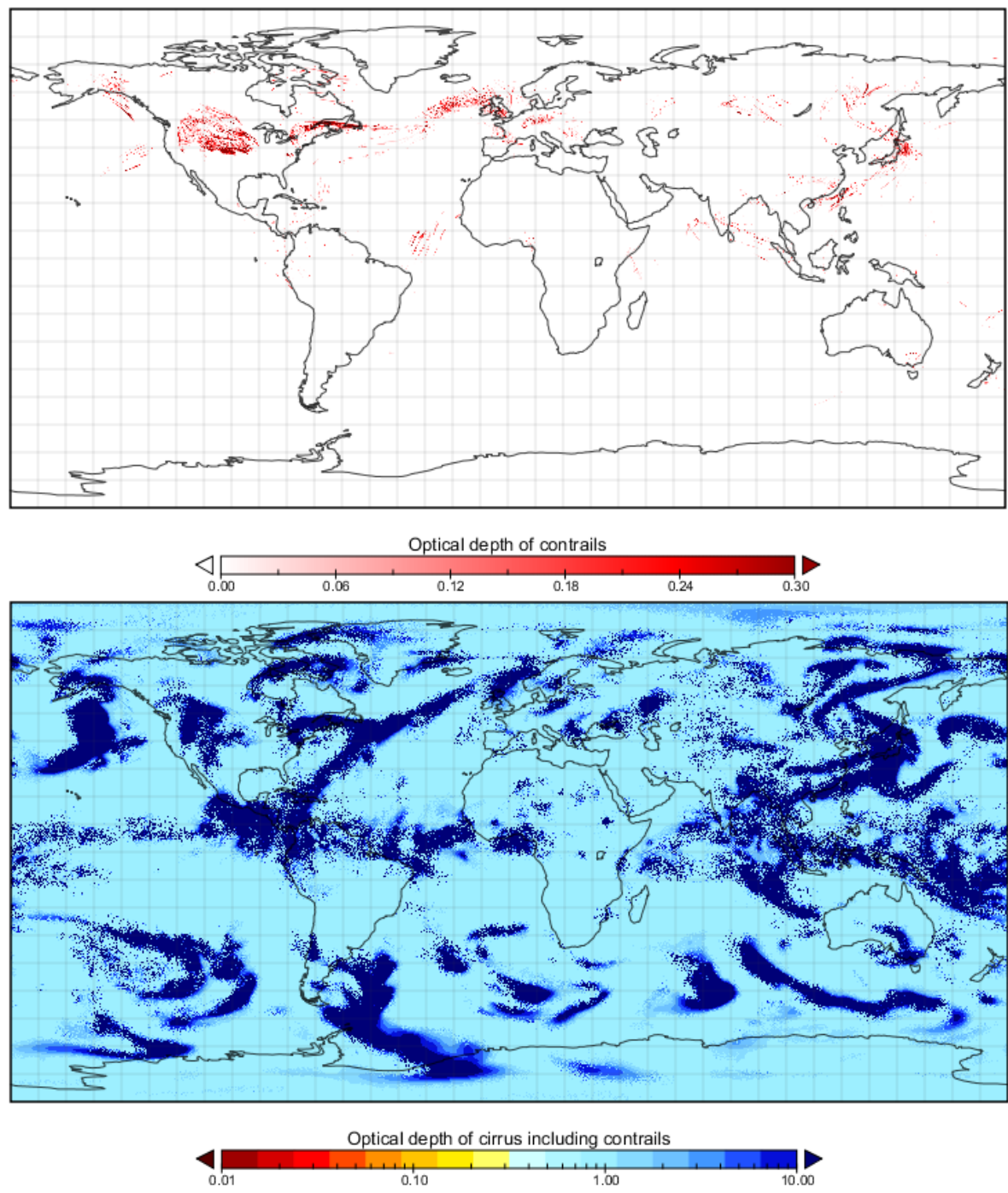

Fig. 11. Optical depth of contrail cover (top) and of cirrus and contrails (bottom) for traffic and time as in Fig. 6. Note, the data include cirrus above $6 \mathrm{~km}$ altitude. The light blue background color indicates zero optical depth.

be expected because the empirical function does not account for different dilution processes (in the jet, wake vortex, and dispersion phases), for aircraft details, and for atmospheric shear and stratification.

CoCiP is applied to compute the dilution of a passive tracer for a flight in a uniform model atmosphere, and for a range of values of shear $S$ and Brunt-Vaisaila frequency $N_{\mathrm{BV}}$, and for three typical commercial aircraft of different sizes, similar to B747, A310, and B737, see Table 3.

Figure 13 shows that the dilution depends on aircraft properties and, in particular, on ambient shear and ambient stratification. As to be expected, the dilution is stronger for smaller aircraft, stronger shear, and weaker stratification. Overall, the order of magnitude agrees with Eq. (16). In fact, CoCiP provides a generalization of this function for given parameters. This agreement is sensitive to various model parameters
Table 2. Critical model parameters.

\begin{tabular}{lll}
\hline Variable & Explanation & Eq. \\
\hline$C_{D 0}=0.5$ & initial wake vortex depth & $(14)$ \\
$C_{r}=0.9$ & volume mean to effective particle radius ratio & $(62)$ \\
$C_{z 1}=0.25$ & wake vortex downwash distance & $(13)$ \\
$E_{A}=1$ & aggregation efficiency & $(52)$ \\
$E_{\text {meso }}=2$ & mesoscale sublimation efficiency & $(55)$ \\
$E_{T}=1$ & turbulent sublimation efficiency & $(49)$ \\
$\mathrm{EI}_{\text {soot }}=3.57 \times 10^{14} \mathrm{~kg}^{-1}$ & soot number emission index & $(17)$ \\
$f_{\text {surv }}=I_{1} / I_{0}$ & survival factor & $(22)$ \\
$f_{T}=0.1$ & sedimentation impact on $D_{\mathrm{V}}$ & $(35)$ \\
$\mathrm{RHI}_{\mathrm{c}}=0.8$ & critical humidity for persistence & $(9)$ \\
$w_{N}^{\prime}=0.1 \mathrm{~m} \mathrm{~s}^{-1}$ & turbulent velocity scale & $(35)$ \\
$\Delta z_{\text {eff }}=2000 \mathrm{~m}$ & effective resolution for shear & $(39)$ \\
$\tau_{c}=0.1$ & optical depth threshold of cover & $(67)$ \\
\hline
\end{tabular}



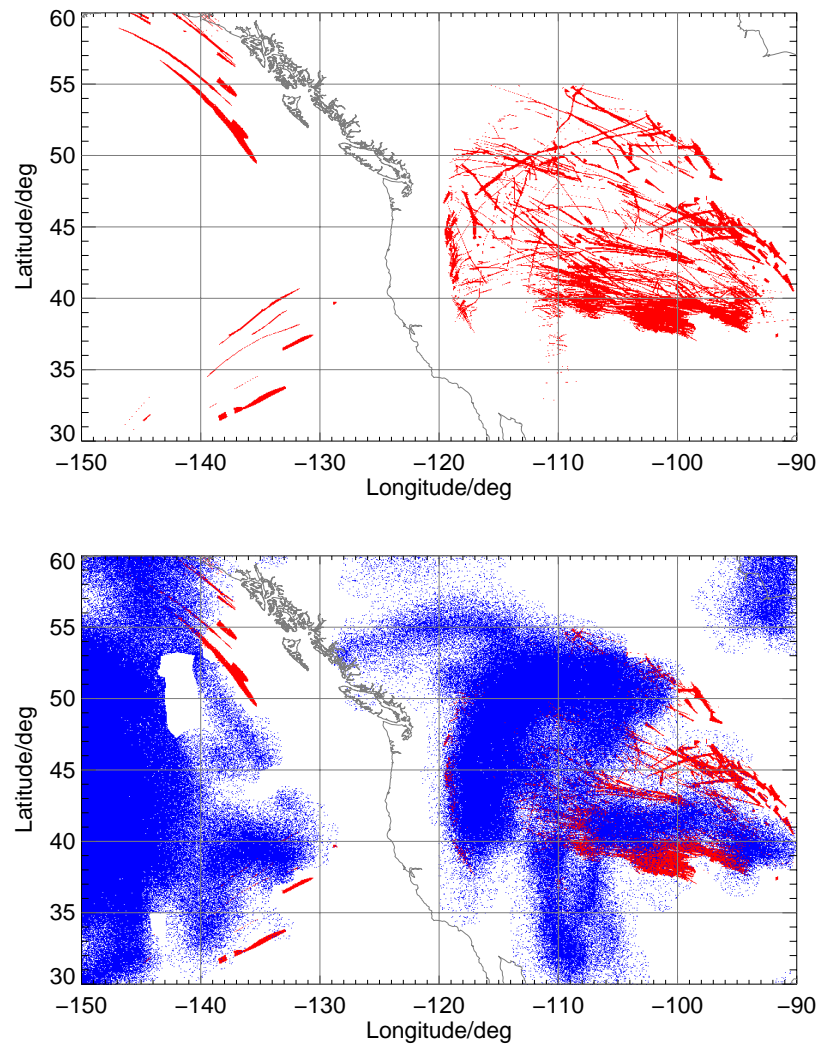

Fig. 12. Distribution of total cirrus-cover composed of natural cirrus and contrail-cirrus for a North American region, with a contrail outbreak. Top: contrail-cirrus pixels (red); bottom: natural cirrus pixels (blue) and contrail-cirrus pixels (red).

listed in Table 2. Smaller initial downwash $\left(C_{z 1}\right)$, vertical diffusivity $\left(w_{N}^{\prime}\right)$, and shear $\Delta z_{\text {eff }}$ reduce dilution. On the other hand, these parameters also control the contrail depth, and larger parameter values may bring our depth results closer to observed values (Freudenthaler et al., 1995).

The computed dilution for integration with two different time steps of either $\Delta t=60 \mathrm{~s}$ or $3600 \mathrm{~s}$ has been found to show differences of about $10 \%$. Hence, the model results are only weakly sensitive to such drastic time step changes. Thus, CoCiP can be applied with rather large time steps. This is essential for computational efficiency in global applications.

\subsection{Aged contrail in comparison with other model results}

CoCiP is designed to simulate contrails with large ages. Comparisons with in-situ measurements for short and mid time-scales will be reported in Sect. 3.4. Here, we describe model results for an artificial case, discuss their plausibility, and compare with results obtained with a more complex $2 \mathrm{~d}$ fluid and microphysics model (Unterstrasser, 2008; Unterstrasser and Gierens, 2010a) for nearly the same case.
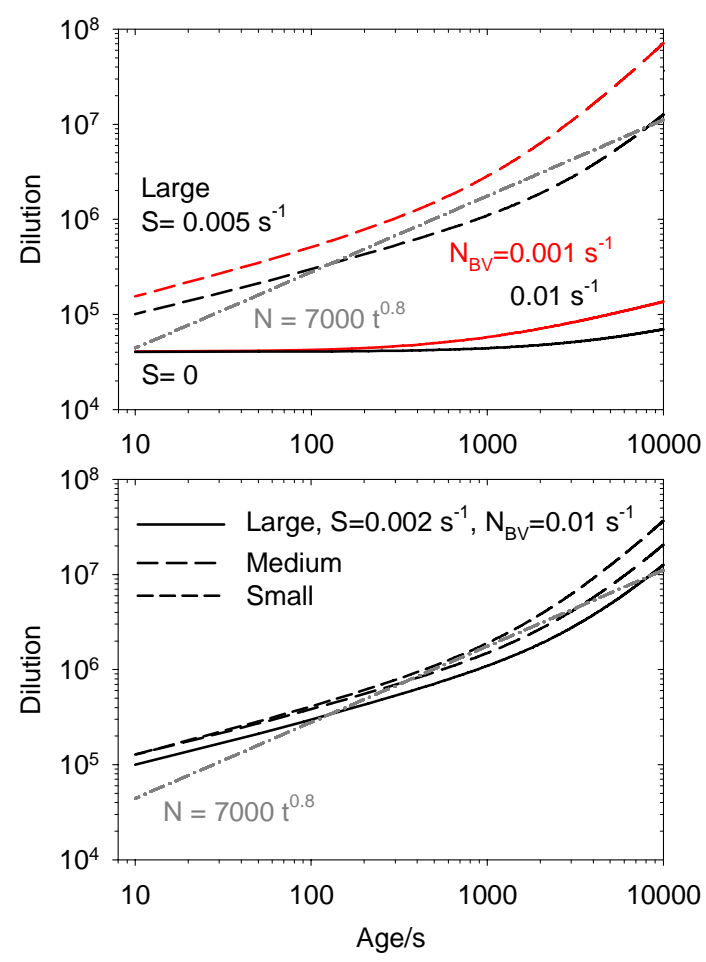

Fig. 13. Bottom: Dilution versus plume age for various aircraft sizes in comparison to an empirical dilution function Eq. (16) for fixed shear $S=0.002 \mathrm{~s}^{-1}$ and Brunt-Vaisaila frequency $N_{\mathrm{BV}}=$ $0.01 \mathrm{~s}^{-1}$. Top: Dilution versus plume age for a large aircraft, for different shear $S$ (full curve: 0 , dashed: $0.005 \mathrm{~s}^{-1}$ ), and different Brunt-Vaisaila frequency $N_{\mathrm{BV}}$ (red: $0.001 \mathrm{~s}^{-1}$, black: $0.01 \mathrm{~s}^{-1}$ ), in comparison to the empirical dilution function.

Table 3. Aircraft parameters used for test cases.

\begin{tabular}{lllll}
\hline & Large & Medium & Small & Unit \\
\hline Type & B747 & A330 & B737 & \\
span $s_{\mathrm{a}}$ & 64.4 & 60 & 34.4 & $\mathrm{~m}$ \\
mass $M_{\mathrm{a}}$ & 310 & 190 & 65 & $\mathrm{Mg}$ \\
fuel flow $m_{\mathrm{F}}$ & 12 & 6.5 & 3 & $\mathrm{~g} \mathrm{~m}^{-1}$ \\
$V_{\mathrm{a}}$ & 250 & 240 & 230 & $\mathrm{~m} \mathrm{~s}^{-1}$ \\
EI $_{\text {soot }}$ & 2.8 & 2.8 & 2.8 & $10^{14} \mathrm{~kg}^{-1}$ \\
$\eta$ & 0.3 & 0.3 & 0.3 & 1 \\
\hline
\end{tabular}

We consider a long-lived contrail, up to $10 \mathrm{~h}$ age, in a static, clear, and uniformly ice-supersaturated atmosphere without vertical motions. Note that the real atmosphere will never be uniform for such a long time period. Both models use the same parameters except that the uniformly supersaturated layer in the $2 \mathrm{~d}$ model is only $1 \mathrm{~km}$ thick, with decreasing humidity above and below (Unterstrasser and Gierens, 2010b). The simulations are performed for a "B747" as listed in Table 3, at $p=250 \mathrm{hPa}, T=217 \mathrm{~K}, N_{\mathrm{BV}}=0.01 \mathrm{~s}^{-1}, S=$ 

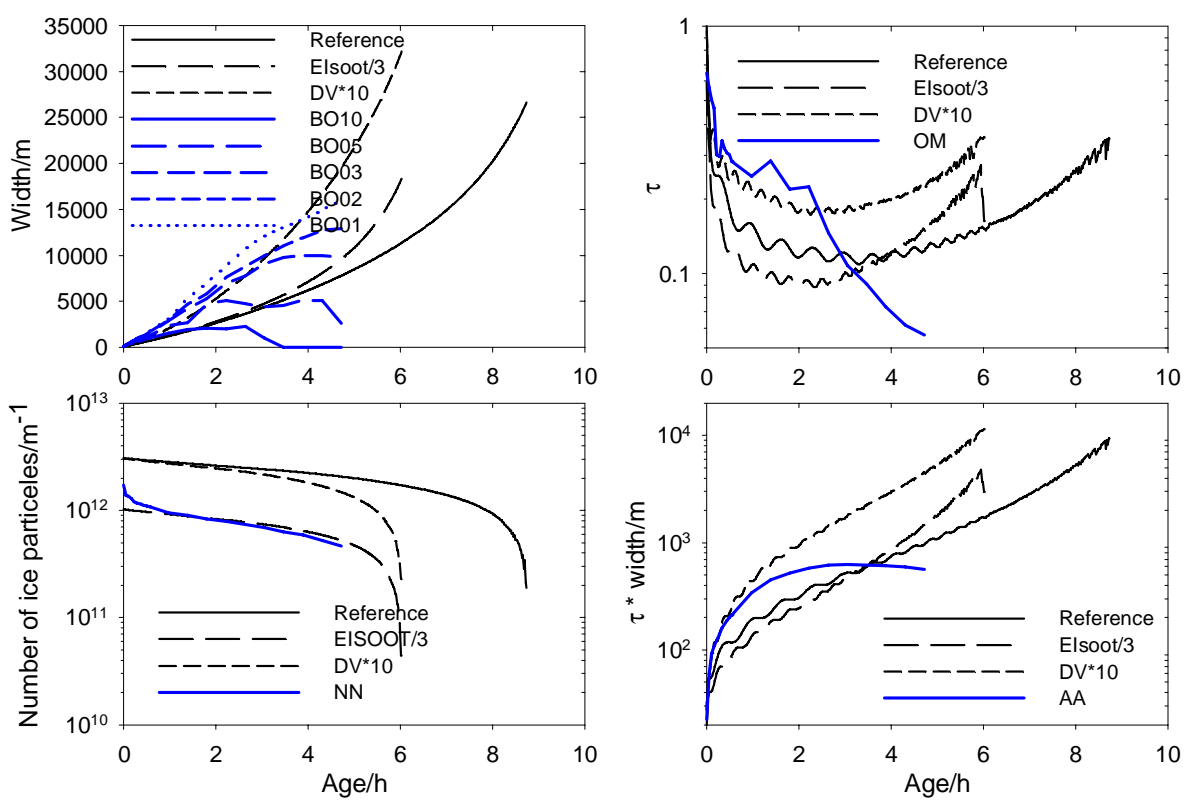

Fig. 14. Comparison of contrail properties versus plume age as computed with CoCiP (black lines) and with a 2d model (blue lines). CoCiP is run for three sets of parameters: reference, reduced soot emissions, and increased vertical diffusivity. Top-left panel: Black: CoCiP width, blue curves: $2 \mathrm{~d}$ model width results BOnn, for various threshold values nn/100 of optical depth. Top-right: black: CoCiP optical depth $\tau$, blue: $2 \mathrm{~d}$ model $\tau$ OM. Bottom-left: black: CoCiP number concentration, blue: $2 \mathrm{~d}$ model number concentration NN. Bottom-right: black: CoCiP product $\tau B$ of optical depth and width, blue: $2 \mathrm{~d}$ integrated optical depth AA.

$0.002 \mathrm{~s}^{-1}$, and $\mathrm{RHi}=1.2$. We vary $E I_{\text {soot }}$ relative to the value given in Table 3 by a factor 3, to study the impact of wake-induced particle losses. In addition, we vary $D_{\mathrm{V}}$ (reference as in Eq. 35), to explain differences between CoCiP and the 2d model, see Figs. 14 and 15.

CoCiP is run in this case with time steps of $150 \mathrm{~s}(5 \mathrm{~s}$ in the $2 \mathrm{~d}$ model). Small wiggles in the CoCiP $\tau$ values are caused by Mie oscillations, Eq. (61). Wiggles at late times are caused by accumulation of approximation errors in the late exponential particle growth phase. Note that the model contains no smoothing which could damp oscillations along waypoint tracks. These wiggles are avoided when using smaller time steps.

Qualitatively, the results of the two models are similar in magnitude and trends, but significant differences are notable. The CoCiP contrail width (Fig. 14 left upper panel) initially increases linearly with time because shear dominates the lateral spread at early times. Note that the width $B$ as defined by the Gaussian plume model is plotted. Smaller plume concentrations spread further away from the contrail center line. In the $2 \mathrm{~d}$ model, the width of the contrail is defined as that part with optical depth larger than given threshold values, as identified in the figure legend (0.01, 0.02, 0.05, and 0.1). Our reference case agrees best with the $2 \mathrm{~d}$ results for optical depth 0.01-0.05. In principle, a similar threshold-width could be computed for the Gaussian profiles in CoCiP. But more important is the product of optical depth with contrail width (or its integral over the lateral coordinate), see Eq. (58), which controls cover and radiative forcing. The right panels of Fig. 14 show $\tau$ and this integral value or product $\tau B$. We see that the two models agree for these important parameters to the order of magnitude. Best agreement is achieved at early times for enhanced vertical diffusivity. Later, the width grows stronger than linear in CoCiP because of increasing depth making shear dispersion more efficient. The thicker depth at later times is mainly caused by sedimentation. For reduced particle emissions, sedimentation becomes important earlier, as expected, so that the width grows more quickly. The thickness also increases for enhanced vertical diffusivity.

The dilution at one hour of age (not shown) is about a factor 4 larger for enhanced vertical diffusivity. Sedimentation effects on dilution are still small $(10 \%-20 \%)$ at this age. For lower diffusivity, the dilution is slightly $(30 \%)$ below, for higher diffusivity, it is 3 times above the empirical function Eq. (16). Hence, both versions and both models are roughly compatible with measured dilution.

The number of ice particles per flight distance $N$ (left lower panel of Fig. 14) remains fairly constant for the first few hours in both models after the wake vortex period. Later, $N$ decreases more quickly in CoCiP. Figure 16 shows that aggregation, Eq. (52), has by far the strongest impact on particle losses in this model. However, the other processes also contribute to these losses.

We note that CoCiP agrees far better with the $2 \mathrm{~d}$ model for reduced particle emissions. This is unexpected, because of the rather low ambient temperature and high humidity. The 

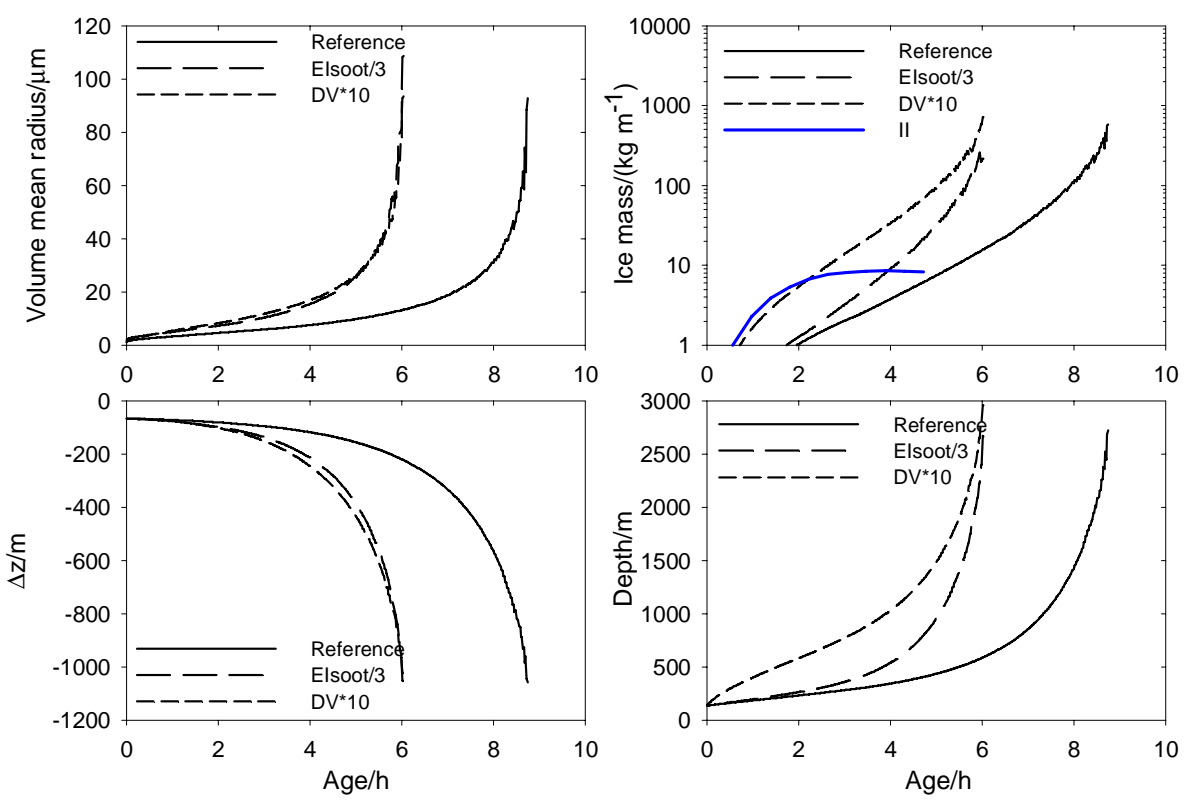

Fig. 15. Same as Fig. 14 for further contrail properties. Top-left panel: Volume mean radius $r$. Top-right: black: Ice mass per contrail length, blue: $2 \mathrm{~d}$ model result II. Bottom-left: Vertical sedimentation displacement $\Delta z$. Bottom-right: Contrail depth $D$.

contrail is formed at conditions far above the SAC threshold $\left(\mathrm{RHi}_{\mathrm{LC}}=3.3, T_{\mathrm{LC}}-T=8.3 \mathrm{~K}\right)$. Therefore, in spite of the size of the aircraft, CoCiP computes large survival fractions of ice particles $\left(f_{\text {surv }}=0.91\right)$, see Fig. 5 . An enhanced vertical diffusivity has a minor impact on $N$. The slow decrease in the first hours is caused by the turbulent decay process simulated according to Eq. (49). Later, the loss by aggregation dominates by far.

The optical depth values of the two models differ most strongly. The CoCiP optical depth comes closer to the $2 \mathrm{~d}$ model result for enhanced vertical diffusivity. Later, the optical depth increases in $\mathrm{CoCiP}$ because the contrail particles start to sediment. Hence, the contrail gets thicker, and collects more humidity from the ambient air, giving larger ice mass, with larger particles and, hence, larger optical thickness values. In contrast, the $2 \mathrm{~d}$ model predicts a decrease in optical depth after $3 \mathrm{~h}$ age. One possible reason for this difference is the limited depth of the supersaturated layer in this example with ice sedimenting out of the layer in the $2 \mathrm{~d}$ model, while CoCiP follows the plume in Lagrangian manner without loss of ice mass. A second reason may result from the assumed ice saturation in the contrail. At late times, the few remaining ice particle may no longer be effective in keeping the contrail at ice saturation, causing less ice water content.

Figure 15 shows further results to explain the contrail dynamics. The left lower panel of this figure shows the small initial downward displacement of the contrail center simulated for the wake vortex phase. Later, this displacement grows and exceeds $1 \mathrm{~km}$ because of sedimentation. The near constant number $N$ of particles share in the growing amount of ice mass from entrained humidity in supersaturated air. Hence, the particles grow in size (see top left panel). Eventually, the particles become large and start sedimenting quickly. In reality the largest particles would sediment first while the smaller ones stay longer at the initial altitude (Jensen et al., 1998a). This separation of particles cannot be simulated with the plume-bulk model. Instead, $\mathrm{CoCiP}$ simulates this process by letting the center of gravity of the contrail sink with time (see left lower panel), similar to what has been deduced from observations (Atlas et al., 2006). At the same time, the contrail depth increases by enhanced vertical diffusivity, see Eq. (35). At contrail ages of $8.7 \mathrm{~h}$ and $6.7 \mathrm{~h}$, for the high and low emission cases, respectively, the particle sizes exceed $100 \mu \mathrm{m}$ in radius, the fall speed exceeds $0.7 \mathrm{~m} \mathrm{~s}^{-1}$ (see lower panel of Fig. 8). Hence, the contrail falls quickly and precipitates at lower altitudes. We note that a reduction of the initial particle number and an increase of vertical diffusivity have similar effects on sedimentation (see mean radius and vertical displacement in the left panels of Fig. 15).

In a rough approximation, the model simulates the effects of fallstreaks which have been observed to form below contrails at high ambient humidity (Schumann, 1994; Heymsfield et al., 1998; Atlas et al., 2006). This process effectively limits the contrail life-cycle at high ambient humidity. Contrails would live longer for lower ambient humidity because of less sedimentation and aggregation. For this reason, the contrail climate impact does not increase linearly with humidity. The example results show also the importance of soot emissions. Reduced soot emissions reduce the number of ice particles but increase their sizes. As a consequence, both the 


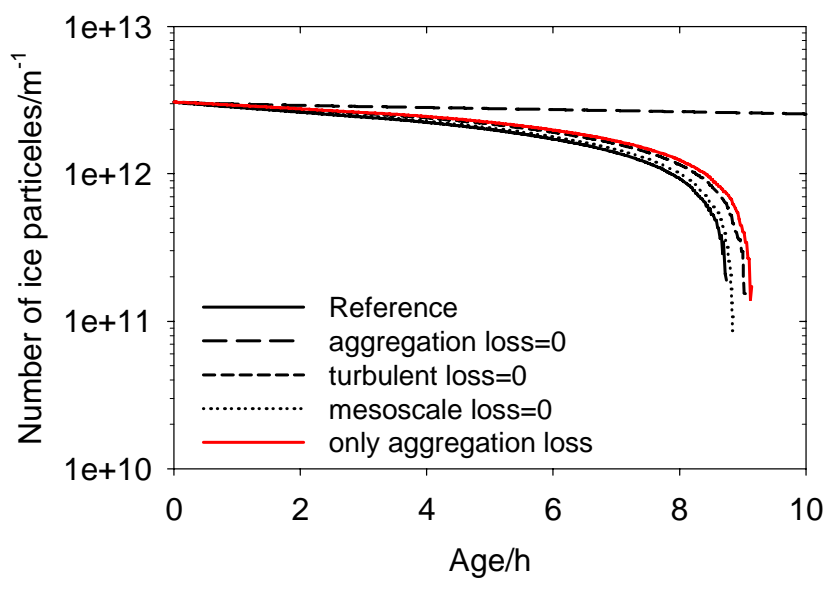

Fig. 16. Ice number per distance: Impact of omitting certain particle loss processes.

optical depth and the lifetime of the contrail get reduced. Both effects reduce the climate impact of contrails.

Although we can explain most of the differences, some differences remain to be understood. It is tempting to take the $2 \mathrm{~d}$ model as benchmark. However, dynamics and microphysics in the $2 \mathrm{~d}$ model also have uncertainties. Obviously, correct representation of turbulent mixing is as important as that of cloud physics. Therefore, in the absence of detailed measurements it is hard to decide which model is closer to the truth.

\subsection{Young and mid-aged contrails in comparison with in-situ observations}

CoCiP is applied to compute the ice bulk properties of contrails for comparison with in-situ measurements for various contrail ages ( $5 \mathrm{~s}-2000 \mathrm{~s})$ behind various aircraft as reported by Schröder et al. (2000), Febvre et al. (2009) and for the CONCERT campaign (Voigt et al., 2010). (We do not expect good agreement for CoCiP for the youngest contrails.) For plume ages larger $150 \mathrm{~s}$, some of the data have been used before to test LES models (Unterstrasser and Gierens, 2010a; Naiman et al., 2011). Also, CoCiP has been tested before by comparison to data for six contrails measured during one measurement flight in CONCERT; here we compare in more detail to the data for a CRJ2 aircraft from that campaign (Voigt et al., 2010).

The observational data are compiled in Table 4 . We replace the reported ice particle concentration $n_{m}$ by $n_{\text {ice }}=$ $\operatorname{IWC} /\left((\pi / 6) \rho_{\text {ice }} d_{\text {mean }}^{3}\right)$, i.e. the value consistent with the reported ice water content IWC and volume mean diameter $d_{\text {mean }}$; both values, partly with large differences, are listed in Table 4. The measured concentrations are uncertain due to variable lower cut-off size of ice particles in the data analysis (Febvre et al., 2009). Some aircraft types and humidity values were unknown and had to be estimated.
Measurements of relative humidity at low ambient temperatures are prone to large uncertainties. The ambient humidity may be estimated for the measured ice water content assuming that the ice water content stems from condensing ambient supersaturation and from the emitted water. Hence, we invert Eq. (17), and use the dilution function, Eq. (16) to obtain

$\mathrm{RHi}_{i}=1+\left[\frac{\mathrm{IWC}}{\rho}-\frac{\mathrm{EI}_{\mathrm{H}_{2} \mathrm{O}}}{7000\left(t_{\text {age }} / t_{\mathrm{s}}\right)^{0.8}}\right] \frac{R_{1} p}{R_{0} p_{\text {ice }}(T)}$.

Table 5 compares the measured relative ice humidity values $\mathrm{RHi}_{m}$ with the computed values $\mathrm{RHi}_{0}$ (for zero engine emissions) and $\mathrm{RHi}_{i}$. We see that the computed values generally are close to the measured ones. The values $\mathrm{RHi}_{0}$ and $\mathrm{RHi}_{i}$ differ considerably for young plumes. This highlights the importance of the water emitted by the aircraft engines in addition to water from ambient supersaturation for young contrails. The measured IWC for the youngest plume cannot be explained this way. The computation for the small age would imply negative ambient humidity. The maximum analyzed humidity values are in a reasonable range, above saturation but below homogeneous nucleation thresholds (Koop et al., 2000), as expected for persistent contrails.

We apply CoCiP for static atmospheres (without subsidence and other changes with time), mostly under ice supersaturated conditions in clear air. During these conditions and ages, the ice loss terms of CoCiP, Eq. (48), though included in these computations, are small. Table 5 lists the model parameters as used for these simulations.

The results are most sensitive to ambient humidity RHi and to dispersion (as a function of ambient shear $S$ ), as to be expected. CoCiP is run with $\mathrm{RHi}=0.9$ for those cases where the table indicates smaller $\mathrm{RHi}_{i}$ values. Unfortunately, also ambient shear, as listed in Table 5, and stratification had to be estimated. In most cases, shear was not reported. Even when the velocity profile was measured (Febvre et al., 2009), where shear was quite large, the effective shear value is difficult to derive because of large variability. Hence, we apply the model with a set of $S$-values, $0,0.001 \mathrm{~s}^{-1}$ and $0.002 \mathrm{~s}^{-1}$.

The results are shown in Fig. 17. The CoCiP results for low and high shear values embrace most of the observed data points. The IWC results for this input are close to the observed values as they should because of adjusted humidity values. The agreement is not perfect, because the dilution in CoCiP depends on the unknown shear, and differs from the empirical dilution function, as expected. With this input, CoCiP reproduces the data as observed in most cases, see Fig. 17. For example, the model comes closest to the observations of Febvre et al. (2009) (ages $150 \mathrm{~s}$ and $900 \mathrm{~s}$ ) for the largest $S$-value. An exception is case A of Schröder et al. (2000), with smallest (5 s) plume age, for which the measured IWC is far less than computed. This may be caused by incomplete condensation in the young contrail.

Because of the uncertainty in ice particle nucleation and in the fraction of ice particles surviving the wake vortex phase, we run $\mathrm{CoCiP}$ with reduced and increased initial particle 
Table 4. Observed contrail properties for comparison with simulations. Refer.: Schr - Schröder et al. (2000) with corresponding aircraft types indicated, Feb - Febvre et al. (2009) for young (y) and aged (a) contrails, and one case of Voigt et al. (2010). For explanation of negative $\mathrm{RHi}_{i}$, see text. Stars* indicate estimated values.

\begin{tabular}{lccccccccccc}
\hline $\begin{array}{l}\text { Refer. } \\
\text { Unit }\end{array}$ & $\begin{array}{c}\text { AC } \\
\text { type }\end{array}$ & $\begin{array}{c}\text { age } \\
\mathrm{s}\end{array}$ & $\begin{array}{c}T \\
{ }^{\circ} \mathrm{C}\end{array}$ & $\begin{array}{c}p \\
\mathrm{hPa}\end{array}$ & $\begin{array}{c}\mathrm{RHi}_{m} \\
1\end{array}$ & $\begin{array}{c}\mathrm{RHi}_{i} \\
1\end{array}$ & $\begin{array}{c}\mathrm{RHi}_{0} \\
1\end{array}$ & $\begin{array}{c}\mathrm{IWC}^{-3} \\
\mathrm{mg} \mathrm{m}^{-3}\end{array}$ & $\begin{array}{c}n_{m} \\
\mathrm{~cm}^{-3}\end{array}$ & $\begin{array}{c}n_{\text {ice }} \\
\mathrm{cm}^{-3}\end{array}$ & $\begin{array}{c}d_{\text {mean }} \\
\mu \mathrm{m}\end{array}$ \\
\hline Schr A & $\mathrm{A} 310$ & 5 & -58 & 238 & 0.53 & -0.35 & 1.02 & 0.26 & 1200 & 2506 & 0.60 \\
Schr AT & ATTAS & 8 & -54 & 287 & 1.02 & 0.45 & 1.12 & 2.80 & 2200 & 3374 & 1.20 \\
Schr B & B757 & 30 & -54 & 262 & 0.85 & 0.83 & 1.04 & 0.91 & 1200 & 2599 & 0.90 \\
Schr A1 & A310 & 70 & -54 & 262 & 0.85 & 1.03 & 1.14 & 3.20 & 1100 & 3033 & 1.30 \\
Voigt CRJ2 & CRJ2 & 90 & -52 & 263 & 1.00 & 0.99 & 1.06 & 1.65 & 125 & 156 & 2.80 \\
Schr B1 & B737 & 120 & -57 & 238 & 0.91 & 1.15 & 1.25 & 3.90 & 2150 & 4700 & 1.20 \\
Schr D & A330* & 135 & -53 & 287 & 1.35 & 1.22 & 1.28 & 7.30 & 1150 & 5540 & 1.40 \\
Feb y & E170 & 150 & -60 & 196 & 1.20 & 0.99 & 1.08 & 0.90 & 68 & 69 & 3.00 \\
Schr A2 & A300* & 200 & -54 & 262 & 0.77 & 1.04 & 1.09 & 2.00 & 290 & 1016 & 1.60 \\
Schr E & A330* & 340 & -55 & 262 & 1.20 & 1.06 & 1.09 & 1.90 & 870 & 1442 & 1.40 \\
Schr B2 & B737 & 600 & -57 & 238 & 0.91 & 1.21 & 1.23 & 3.70 & 180 & 963 & 2.00 \\
Schr F & Falcon & 700 & -55 & 262 & 1.20 & 1.12 & 1.14 & 2.90 & 130 & 652 & 2.10 \\
Schr O & A330* & 800 & -54 & 274 & 1.40 & 1.13 & 1.15 & 3.40 & 95 & 665 & 2.20 \\
Feb a & E170 & 900 & -60 & 196 & 1.20 & 1.07 & 1.09 & 1.00 & 19 & 20 & 4.70 \\
Schr U & B737* & 2000 & -56 & 238 & $1.10 *$ & 1.21 & 1.22 & 3.90 & 11 & 23 & 7.00 \\
\hline
\end{tabular}

Table 5. Input and results of contrail simulation. The last three columns list the CoCiP output. References as in Table 4.

\begin{tabular}{|c|c|c|c|c|c|c|c|c|c|c|c|}
\hline $\begin{array}{l}\text { Refer. } \\
\text { Unit }\end{array}$ & $\begin{array}{l}\text { age } \\
\mathrm{s}\end{array}$ & $\begin{array}{l}\eta \\
1\end{array}$ & $\begin{array}{c}V_{\mathrm{a}} \\
\mathrm{m} \mathrm{s}^{-1}\end{array}$ & $\begin{array}{l}M_{\mathrm{a}} \\
\mathrm{Mg}\end{array}$ & $\begin{array}{c}m_{\mathrm{F}} \\
\mathrm{g} \mathrm{m}^{-1}\end{array}$ & $\begin{array}{l}s_{\mathrm{a}} \\
\mathrm{m}\end{array}$ & $\begin{array}{c}\mathrm{EI}_{\text {soot }} \\
10^{14} \mathrm{~kg}^{-1}\end{array}$ & $\begin{array}{c}S \\
10^{-3} \mathrm{~s}^{-1}\end{array}$ & $\begin{array}{c}\mathrm{IWC}_{c} \\
\mathrm{mg} \mathrm{m}^{-3}\end{array}$ & $\begin{array}{c}n_{c} \\
\mathrm{~cm}^{-3}\end{array}$ & $\begin{array}{l}d_{c} \\
\mu \mathrm{m}\end{array}$ \\
\hline Schr A & 5 & 0.33 & 230 & 120 & 4.40 & 43.9 & 3 & 0 & 13.50 & 3532 & 2.00 \\
\hline Schr AT & 8 & 0.17 & 177 & 18 & 1.00 & 21.5 & 10 & 1 & 2.55 & 3910 & 1.11 \\
\hline Schr B & 30 & 0.33 & 240 & 95 & 4.31 & 38.0 & 3 & 0 & 14.46 & 3960 & 1.97 \\
\hline Schr A1 & 70 & 0.33 & 230 & 120 & 5.06 & 43.9 & 3 & 0 & 15.26 & 3453 & 2.10 \\
\hline Voigt CRJ2 & 90 & 0.26 & 210 & 52 & 1.30 & 21.2 & 3 & 2 & 0.67 & 230 & 1.82 \\
\hline Schr B1 & 120 & 0.33 & 230 & 60 & 2.82 & 34.3 & 3 & 0 & 17.57 & 3582 & 2.17 \\
\hline Schr D & 135 & 0.33 & 230 & 190 & 6.50 & 60.3 & 3 & 0 & 15.09 & 2298 & 2.39 \\
\hline Feb y & 150 & 0.33 & 220 & 30 & 1.85 & 26.0 & 3 & 2 & 0.79 & 210 & 1.99 \\
\hline Schr A2 & 200 & 0.33 & 230 & 140 & 6.22 & 44.8 & 3 & 1 & 2.46 & 331 & 2.49 \\
\hline Schr E & 340 & 0.33 & 230 & 190 & 6.50 & 60.3 & 3 & 0 & 10.27 & 2143 & 2.15 \\
\hline Schr B2 & 600 & 0.33 & 230 & 60 & 2.82 & 34.3 & 3 & 1 & 4.07 & 121 & 4.13 \\
\hline Schr F & 700 & 0.33 & 180 & 10 & 0.57 & 16.3 & 3 & 1 & 2.95 & 49 & 4.99 \\
\hline Schr O & 800 & 0.33 & 230 & 190 & 6.50 & 60.3 & 3 & 1 & 3.83 & 122 & 4.03 \\
\hline Feb a & 900 & 0.37 & 220 & 30 & 1.85 & 26.0 & 3 & 2 & 1.07 & 53 & 3.48 \\
\hline Schr U & 2000 & 0.33 & 230 & 60 & 2.82 & 34.3 & 3 & 2 & 4.14 & 27 & 6.85 \\
\hline
\end{tabular}

emissions. For this purpose, we simply multiply the soot emission index with factors 0.5 or 2 . We found that the agreement gets worse when simulating a smaller survival ratio by reducing the particle emissions. The agreement gets also worse for enhanced particle emissions, which should provide better results if ice particle formation, e.g. in volatile particles, is important. This result is in part a consequence of the selected shear values. Nevertheless, the test shows that the data are consistent with the model for the selected parameter values.

Finally, we test again the sensitivity to the vertical diffusivity. We changed $D_{\mathrm{V}}$ by factors 0.5 and 10 compared to the reference case. For plume ages below $3 \mathrm{~min}$, the impact of $D_{\mathrm{V}}$ is minor, as expected. For larger plume ages, e.g. case $\mathrm{U}$, an increase in $D_{\mathrm{V}}$ by a factor 10 causes more dilution and considerably (factor 7) smaller particle concentrations, therefore. The ice water content increases slightly (by $5 \%$ ) 

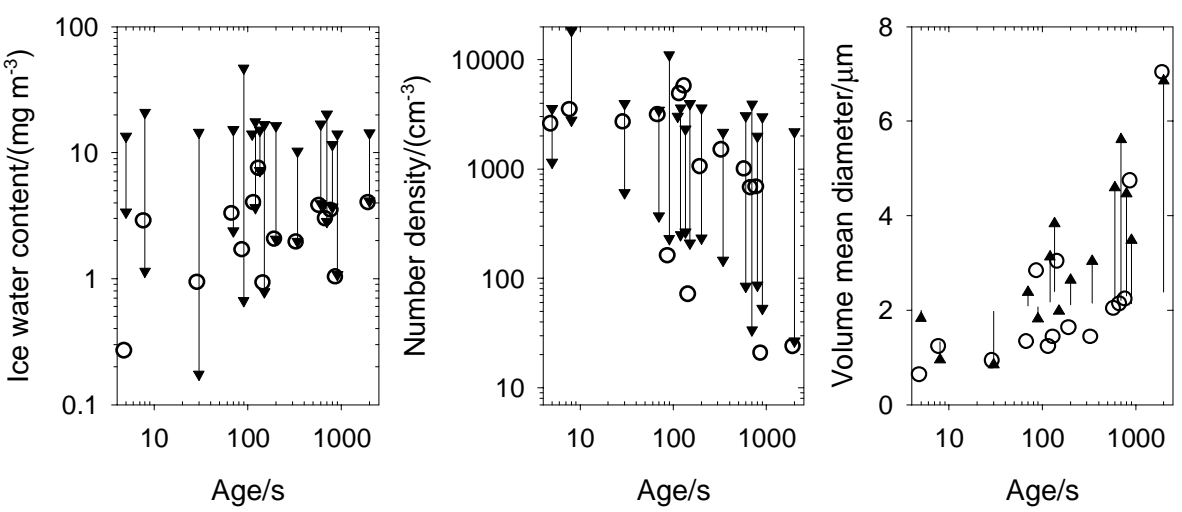

Fig. 17. Comparison of CoCiP results (lines with triangles, pointing towards increasing shear in the range $0-0.002 \mathrm{~s}^{-1}$ ), with observations (open circles) versus contrail age. From left to right: Ice water content, ice particle number concentration, volume mean ice particle diameter.

and the particles become a factor 2 larger in diameters. However, the agreement with observations deteriorates when $D_{\mathrm{V}}$ gets increased compared to the reference case. Hence, the diffusivity parameterization should stay unchanged.

It appears that the bulk microphysics model used in CoCiP is sufficient to explain the contrail properties consistent with the observations. The agreement between results and observations is at least as good for CoCiP as it is for LES models (Unterstrasser and Gierens, 2010a; Naiman et al., 2011). To some extent this implies that the set of measurements are not complete (e.g. for shear) or accurate enough (e.g. for humidity) to provide rigorous constrains. However, the comparison shows that the model gives a fair representation of the fraction of particles surviving the early wake vortex. There is no hint for important ice nucleation in volatile particles in these cases.

\subsection{Further discussion}

The results presented provide support for the usefulness of the model. However, because of the large number of meteorological and traffic input parameters and the simplifying assumptions used in the model, further parameter and validation studies are to be performed. For example, the model results depend critically on the quality of the NWP humidity input data. These have been shown to be reasonable (Tompkins et al., 2007; Lamquin et al., 2012). It also depends on the parameter $\mathrm{RHi}_{c}$ used in the troposphere in the NWP model, and on further parameters, such as the type of interpolation (in absolute or relative humidity, Appendix A2). These parameters should be varied and tested by direct comparisons of measured and computed contrail results. The single Gaussian plume model may need improvements for the wake vortex phase. Two plumes instead of one may be used to simulate the primary and secondary vortex parts (Gerz and Ehret, 1996; Lewellen and Lewellen, 1996) separately. This would allow simulating deeper sinking of the primary wake vortex part without overestimate of dilution. A deeper sinking would reduce the survival factor of ice particles in the wake phase. The model assumes ice saturation and equilibrium inside the contrail, which may cause significant errors for low ice particle concentrations. The model in the present form does not account for radiative heating which may be essential for thick long-lived contrails. The contrails are simulated in this paper without feedback among each other or with the background meteorology. Narrow contrails fill only a small fraction of the grid volume of a NWP model, so that the change of grid cell mean humidity by few contrails is small. Larger changes may occur for overlapping contrails and for many contrails in one grid cell (Burkhardt and Kärcher, 2011). Coupling of the model with the NWP model is an option to test the importance of this feedback. The bulk model does not account for the dependency of the equilibrium vapor pressure on the ice surface tension (Kelvin effect) which is important for very small ice particles (Pruppacher and Klett, 1997). A recent study suggests particle losses due to larger crystals scavenging water vapor from smaller ones because of the Kelvin effect (Lewellen, 2012). More research is required to improve understanding and modeling of the microphysics controlling ice crystal number losses (Sect. 2.12). Theses losses are critical for the simulated contrail lifetime and climate impact. As explained, only few data exist to test the lifetimes of contrails. This is also true for cirrus and ice supersaturated regions in general. CoCiP may serve as a model framework for testing alternative ice bulk models in relationship to contrail observations.

\section{Conclusions}

The contrail physics model implemented in the "Contrail Cirrus Prediction Tool" (CoCiP) has been described in detail. The model has been applied successfully for a global case and for individual contrails in comparison with other model results and in-situ measurements.

The Lagrangian Gaussian contrail model treats the lifecycle of contrails with bulk contrail ice physics, containing several simplifying assumptions (see Sect. 3.5). The model is 
efficient in treating mixing and cloud processes quasi analytically. The numerical scheme is unconditionally stable and guarantees positive definite solutions. The simulation of an individual contrail along a flight track with hundreds of waypoints with maximum lifetime of order a day, requires far less than a second computing time on a laptop. This makes contrail simulations for a large fleet of aircraft feasible.

The model accounts for the influence of aircraft properties and ambient meteorology. This includes standard contrail formation thresholds, advection, turbulent mixing, and ice mass formation from emitted and ambient humidity, with ice crystal number depending on the number of soot particles emitted. The model includes simple approximations for ice particle survival in the adiabatically sinking wake vortices, and particle losses in aged contrails. These model parts may need to be further improved when comparing to further observations. Moreover, we note the strong sensitivity of the model results to turbulent diffusivities. This calls for further refinement of the parameters for this purpose, e.g. kinetic energy and dissipation rates from NWP input data.

CoCiP provides a generalization of an often used simple dilution function derived previously from measurements (Schumann et al., 1998). The dilution of passive tracers from aircraft engines in the wake vortex and dispersion phases depends weakly on aircraft properties and rather strongly on shear and stratification in the ambient atmosphere.

The model reproduces measured contrail properties for given aircraft type in terms of ice water content, crystal number concentration, and mean crystal diameters, in contrails of about $100 \mathrm{~s}-2000 \mathrm{~s}$ age. Comparable measurements in isolated single contrails at larger ages are missing.

The CoCiP model constrains the ambient relative humidity at the time of contrail formation, which is difficult to measure. It is also used to estimate the ambient wind shear controlling contrail dispersion. The comparison supports the assumption that the number of ice particles in the contrail at several minutes plume age is mainly determined by the number of soot particles emitted by the aircraft engines. Turbulence and cloud physics are equally important for explaining observed contrail dynamics.

Particle loss processes, in particular sedimentation in warm ice supersaturated air masses, control the contrail lifetime. Such contrails end with fallstreaks. Contrails persist shorter for smaller soot particle emissions. Aged contrails contribute most to climate change because of largest product of optical depth and width. More data are needed to constrain the maximum ages of contrails and their final dissipation processes.

We have shown an example with contail-cirrus outbreaks. The linear shape of young contrails gets lost in this model by overlap with other contrails and cirrus. The global-mean contrail-cirrus cover is small, but reaches $100 \%$ regionally at scales of a few $100 \mathrm{~km}$, depending nonlinearly on traffic density and threshold values for cloud detectability.

\section{Appendix A}

\section{Model details}

\section{A1 Gaussian area integral}

The area integral, Eq. (3), can be evaluated using a principal axis transformation

$\mathbf{x}^{T} \sigma^{-1} \mathbf{x}=\mathbf{z}^{T} \mathbf{X}^{-1} \Lambda \mathbf{X z}$.

With eigenvalues $\Lambda$, defined by

$\sigma^{-1} \mathbf{X}=\Lambda \mathbf{X}, \Lambda=\operatorname{diag}\left(\lambda_{1}, \lambda_{2}\right)$,

and substitution $\mathbf{y}=\mathbf{X z}$,

we have

$A=\iint \exp \left[-(1 / 2) \mathbf{y}^{T} \Lambda \mathbf{y}\right] d \mathbf{y}$.

With,

$\mathbf{y}^{T} \Lambda \mathbf{y}=y_{1} \lambda_{1} y_{1}+y_{2} \lambda_{2} y_{2}$,

we obtain

$A=\iint \exp \left[-(1 / 2)\left(y_{1} \lambda_{1} y_{1}+y_{2} \lambda_{2} y_{2}\right)\right] d y_{1} d y_{2}$

$=\left[\int \exp \left[-(1 / 2) \lambda_{1} y_{1}^{2}\right] d y_{1}\right]\left[\int \exp \left[-(1 / 2) \lambda_{2} y_{2}^{2}\right] d y_{2}\right]$.

With (Abramowitz and Stegun, 1964)

$\int_{-\infty}^{\infty} \exp \left[-(1 / 2) \lambda y^{2}\right] d y=(2 \pi / \lambda)^{1 / 2}$,

and

$\lambda_{1} \lambda_{2}=\operatorname{det}\left(\sigma^{-1}\right)=1 / \operatorname{det}(\sigma)$,

we obtain the result as given in Eq. (3),

$A=2 \pi[\operatorname{det}(\sigma)]^{1 / 2}$.

In previous applications of the Konopka model, the first factor 2 in Eq. (3) was often missing.

\section{A2 Interpolation aspects}

Interpolation is performed, e.g. for a function $F_{i, j}$ at discrete grid-points $(i, j)$ in a Cartesian $x-y$-plane, with equidistant grid spacings $\Delta x$ and $\Delta y$, and mid-cell coordinates $x_{i}, y_{j}$, using bilinear interpolation:

$F(x, y)=\frac{1}{4}\left(g_{1} F_{i, j}+g_{2} F_{i+1, j}+g_{3} F_{i+1, j+1}+g_{4} F_{i, j+1}\right)$, where $g_{1}=(1-\gamma)(1-\eta), g_{2}=(1+\gamma)(1-\eta), g_{3}=(1+$ $\gamma)(1+\eta), g_{4}=(1-\gamma)(1+\eta)$, with $\gamma=\left(x-x_{i}\right) 2 / \Delta x, \eta=$ $\left(y-y_{j}\right) 2 / \Delta y$.

Relative (RHi) and absolute $(q)$ humidity, for given temperature $T$ and pressure $p$, are nonlinearly related by saturation pressure $p_{\text {ice }}(T)$ and $q=\operatorname{RHi}\left(p_{\text {ice }}(T) / p\right) R_{0} / R_{1}$. Interpolation of humidity may be performed in NWP input in either variable. Both variants introduce up to $7.2 \%$ errors (either in RHi or in $q$ ) for $50 \mathrm{hPa}$ pressure intervals, see Fig. A1. CoCiP has been coded in both variants, and the differences have been found to be important because of strong sensitivity to relative humidity in the SAC and to absolute humidity in the ice water content and optical depth. The results in this paper were computed with interpolation in q, partly (Sects. 3.23.4) with fine vertical resolution. 


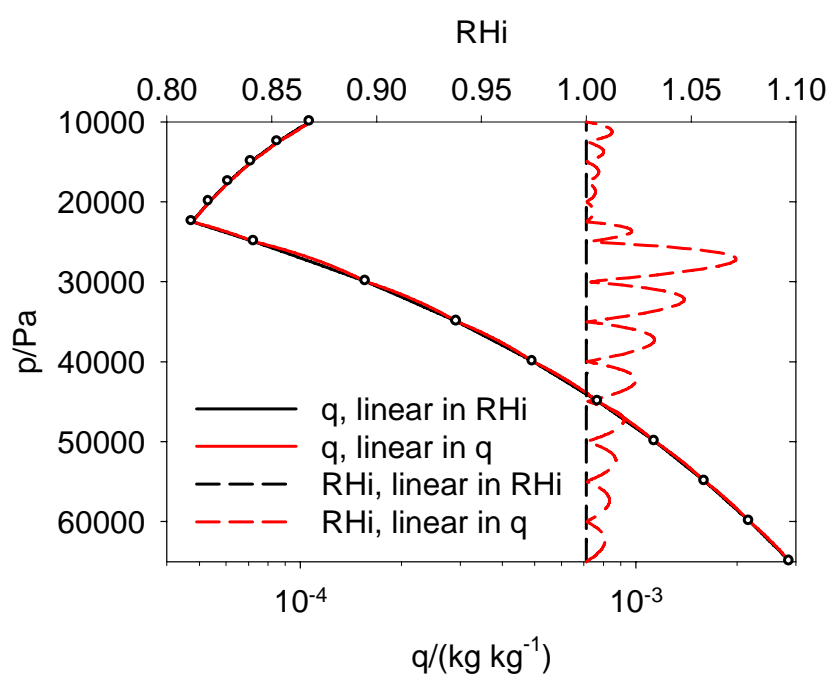

Fig. A1. Illustration of the difference in local relative humidity over ice (RHi) caused by either interpolating in absolute or relative humidity. Full curves: Absolute humidity $q$ versus pressure altitude $p$ for the ICAO standard atmosphere with RHi $=100 \%$. The dots represent the discrete pressure values as given in the NWP input. Data are constructed for this example (as in the ECMWF data) with $50 \mathrm{hPa}$ intervals below $250 \mathrm{hPa}$ and $25 \mathrm{hPa}$ above that altitude. The full black line represents the interpolation in absolute humidity. The red full line represents the interpolation in relative humidity. Dashed curves: RHi versus pressure altitude $p$. The dashed black line represents RHi computed by linear interpolation in relative humidity. The dashed red line represents RHi computed by linear interpolation in absolute humidity. The difference in RHi (up to 0.07) between the red and black dashed lines is a consequence of different interpolation methods either between discrete $q$ or RHi values.

\section{A3 Saturation pressure}

Saturation pressure over liquid and ice water surfaces, $p_{\text {liq }}$ and $p_{\text {ice }}$, is approximated as in Sonntag (1994):

$$
\begin{gathered}
p_{\text {liq }}(T)= \\
100 \exp [-6096.9385 / T+16.635794-0.02711193 T \\
\left.+1.67395210^{-5} T^{2}+2.433502 \ln (T)\right] \\
p_{\text {ice }}(T)= \\
100 \exp [-6024.5282 / T+24.7219+0.010613868 T \\
\left.-1.319882510^{-5} T^{2}-0.49382577 \ln (T)\right] .
\end{gathered}
$$

The equation for ice saturation agrees better than $1 \%$ with approximations recommended by Murphy and Koop (2005) at temperatures down to $-100^{\circ} \mathrm{C}$. Somewhat larger differences are found for liquid saturation.

\section{A4 Altitude in the standard atmosphere}

In the ICAO standard atmosphere $\left(T=15^{\circ} \mathrm{C}\right.$ at the surface, decreasing linearly with constant laspe rate $d T / d z=$
$-6.5 \mathrm{~K} \mathrm{~km}^{-1}$ up to $T=-56.5^{\circ} \mathrm{C}$ at $11 \mathrm{~km}$ altitude, and constant above), the flight level $z$ is converted to a static pressure $p$, for $p$ in $\mathrm{Pa}$ and $z$ in $\mathrm{m}$ (ICAO, 1964): In the troposphere $(z<11000 \mathrm{~m}, p>22632 \mathrm{~Pa})$,

$$
\begin{gathered}
p=101325\left(1-2.2557710^{-5} z\right)^{5.25589}, \\
z=44330.8\left[1-(p / 101325)^{0.190263}\right] .
\end{gathered}
$$

Otherwise,

$$
\begin{gathered}
p=22632 \exp \left[-1.5768910^{-4}(z-11000)\right], \\
z=[11000-6341.62 \ln (p / 22632)] .
\end{gathered}
$$

\section{A5 Contrail formation conditions}

The Schmidt-Appleman criterion (SAC) requires computing a threshold temperature $T_{\mathrm{LC}} \leq T_{\mathrm{LM}}$ depending on ambient relative humidity over liquid water $U=\operatorname{RHi} p_{\text {ice }}(T) / p_{\text {liq }}(T)$ and the steepness $G$ of the mixing line, see Eq. (11). The exact solutions for $T_{\mathrm{LM}}$ and $T_{\mathrm{LC}}$ follow implicitly from (Schumann, 1996)

$p_{\text {liq }}^{\prime}\left(T_{\mathrm{LM}}\right)=G$

and

$T_{\mathrm{LC}}=T_{\mathrm{LM}}-\frac{p_{\mathrm{liq}}\left(T_{\mathrm{LM}}\right)-U p_{\mathrm{liq}}\left(T_{\mathrm{LC}}\right)}{G}$.

For $U \ll 1$ and $U=1$, and for given $T_{\mathrm{LM}}$, e.g. from Eq. (10), an explicit solution for the threshold temperature $T_{\mathrm{LC}}$ follows from:

$T_{\mathrm{LC}}=T_{\mathrm{LM}}-(1-U) p_{\mathrm{liq}}\left(T_{\mathrm{LM}}\right) / G$ and $T_{\mathrm{LC}}=T_{\mathrm{LM}}$,

respectively. So far, no explicit approximation was available for $T_{\mathrm{LC}}$, for $0<U<1$.

A solution can be found by solving Eq. (A6) iteratively for given humidity $U$ and value of $G$. To start the iteration we use a quadratic Taylor series of $p_{\text {liq }}\left(T_{\mathrm{LM}}\right)$ around $T_{\mathrm{LM}}$ with $\Delta T=T_{\mathrm{LM}}-T_{\mathrm{LC}}$,

$$
\begin{gathered}
p_{\text {liq }}\left(T_{\mathrm{LC}}\right) \approx \\
p_{\text {liq }}\left(T_{\mathrm{LM}}\right)-\Delta T p^{\prime \text { liq }}\left(T_{\mathrm{LM}}\right)+\frac{\Delta T^{2}}{2} p_{\text {liq }}^{\prime \prime}\left(T_{\mathrm{LM}}\right) .
\end{gathered}
$$

With this series, Eq. (A6) implies

$T_{\mathrm{LC}}=T_{\mathrm{LM}}+A-\sqrt{A^{2}+2 B}$.

Schumann (1996) gives expressions for $A$ and $B$ requiring to calculate the second derivative of $p_{\text {liq }}^{\prime \prime}\left(T_{\mathrm{LC}}\right)$ with respect to $T$. (Eq. 34 of this reference contains a mistake: $U^{2}$ should be replaced by $U$ (Ferrone, 2011).) The second derivative of $p_{\text {liq }}(T)$ can be approximated by (e.g. Ferrone, 2011)

$p_{\mathrm{liq}}^{\prime \prime}\left(T_{\mathrm{LM}}\right) \approx \frac{2 p_{\mathrm{liq}}\left(T_{L 0}\right) G^{2}}{p_{\mathrm{liq}}\left(T_{\mathrm{LM}}\right)}$. 


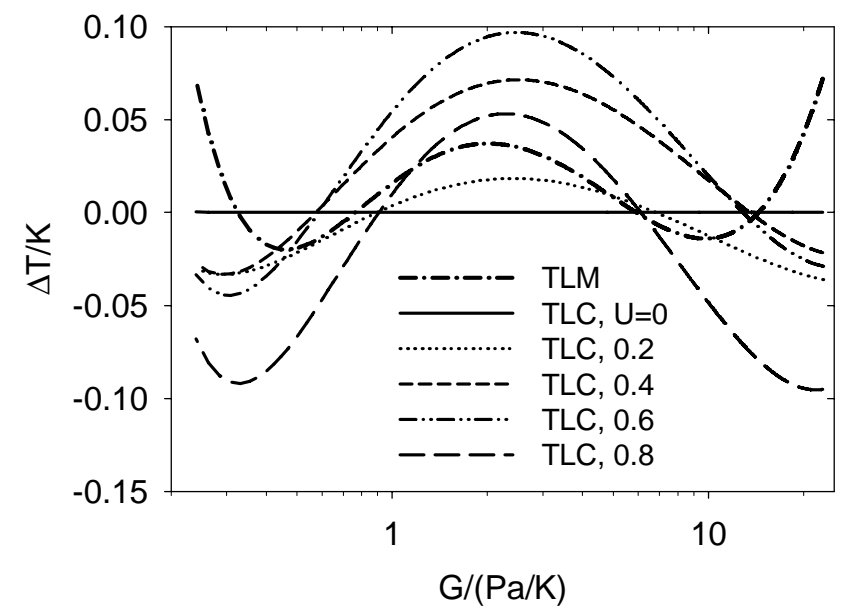

Fig. A2. Differences between fits (Eqs. 10 and A16) and exact solutions for (bold curve:) contrail threshold temperature at liquid saturation $T_{\mathrm{LM}}=T_{\mathrm{LC}}(U=1)$ and (thin cuves:) for $T_{\mathrm{LC}}(U)$ at various values of liquid relative humidity $U$.

This gives

$A=\frac{(1-U) p_{\mathrm{liq}}\left(T_{\mathrm{LM}}\right)\left(T_{\mathrm{LM}}-T_{L 0}\right)}{2 U p_{\mathrm{liq}}\left(T_{L 0}\right)}$,

$B=A\left(T_{\mathrm{LM}}-T_{L 0}\right), T_{L 0}=T_{\mathrm{LM}}-p_{\mathrm{liq}}\left(T_{\mathrm{LM}}\right) / G$.

The approximate result, Eq. (A9), deviates from the exact solution with maximum errors $0.3 \mathrm{~K}$ at humidity $U \approx 0.92$, nearly independent of $G$. Higher accuracy is achieved with a few Newton iteration steps:

$F=p_{\text {liq }}\left(T_{\mathrm{LM}}\right)-G\left(T_{\mathrm{LM}}-T_{\mathrm{LC}}\right)-U p_{\mathrm{liq}}\left(T_{\mathrm{LC}}\right)$,

$F^{\prime}=G-U p^{\prime} \mathrm{liq}_{(}\left(T_{\mathrm{LC}}\right)$

$\Delta T=F / F^{\prime}, T_{\mathrm{LC}}=T_{\mathrm{LC}}-\Delta T$,

until $\Delta T$ is less than about $0.001 \mathrm{~K}$.

The iteration is delicate because it requires the first and second derivatives of the saturation pressure and because $T_{\mathrm{LC}}$ varies smoothly for low $U$ but suddenly increases near $U=1$, see Fig. A3. For these reasons, it was not easy to find better fitting approximations. The analytical solutions for a third order Taylor series instead of Eq. (A8) were found to be sensitive to round-off errors.

A suitable fit extends Eq. (A7):

$T_{\mathrm{LC}}=T_{\mathrm{LM}}-(1-U) \frac{p_{\mathrm{liq}}\left(T_{\mathrm{LM}}\right)}{G}-\Delta T_{c}$,

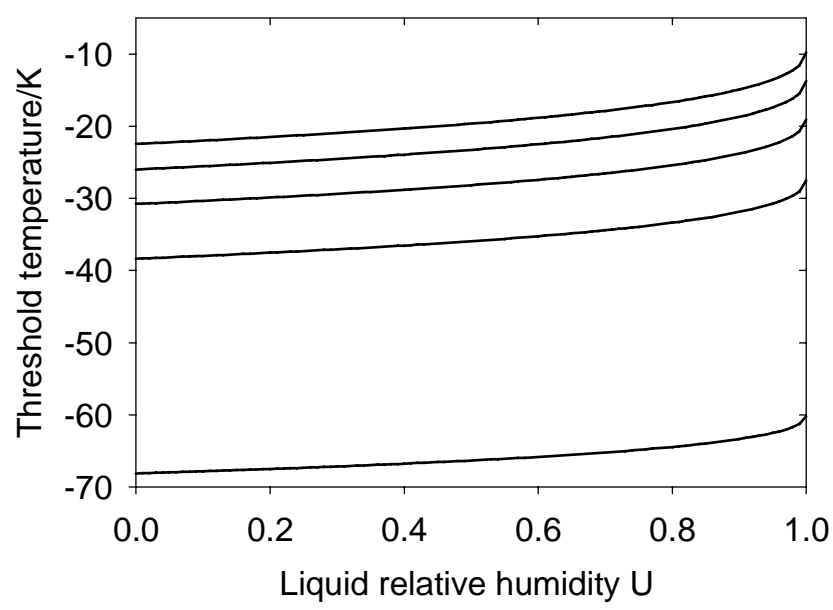

Fig. A3. Threshold temperature $T_{\mathrm{LC}}$ versus liquid relative humidity for 5 values of $G$, equidistant within $(0.24-23) \mathrm{Pa} \mathrm{K}^{-1}$, increasing from bottom to top.

with a correction $\Delta T_{c}$ which is zero for $U=0,1$ and increases sharply near $U=1$,

$\Delta T_{c}=F_{1} U\left[W-F_{2}(1-W)\right]$,

with

$F_{1}=x_{1}+x_{3} \ln (G), W=\left(1-U^{2}\right)^{x_{2}}$,

$F_{2}=\left[\frac{1}{4}-\left(U-\frac{1}{2}\right)^{2}\right]^{4}$.

The fit uses three fit coefficients $x_{1}=5.686, x_{2}=0.3840$, $x_{3}=0.6594$. The maximum error is $0.13 \mathrm{~K}$ over the full range $0 \leq U \leq 1,0.24 \mathrm{PaK}^{-1}<G<23 \mathrm{PaK}^{-1}$ (see Fig. A2). It is efficient because $T_{\mathrm{LC}}$ is computed with just one evaluation of $p_{\text {liq }}\left(T_{\mathrm{LM}}\right)$.

\section{A6 Contributions of emitted heat and water and latent heat release}

Engine water emissions increase the relative humidity over ice within the plume as a function of dilution, $\triangle \mathrm{RHi}=$ $\mathrm{EI}_{\mathrm{H}_{2} \mathrm{O}} R_{1} p /\left(R_{0} p_{\text {ice }}(T) N_{\text {dil }}(t)\right)$. The dilution can be estimated with Eq. (16). Figure A4 shows the change in relative humidity versus time after emission for selected values of ambient temperature $T(200 \mathrm{~K}$ and $240 \mathrm{~K})$ and pressure $p(100 \mathrm{hPa}$ and $400 \mathrm{hPa})$. The water vapor emission contributes considerably to relative humidity at low temperatures (where ambient air contains little water vapor). The contribution increases linearly with pressure because the plume mass, over which the emissions get spread, increases with pressure. Hence, the emitted water vapor is important (order $5 \%$ or larger) for times up to about $1 \mathrm{~h}$ at low temperatures near 


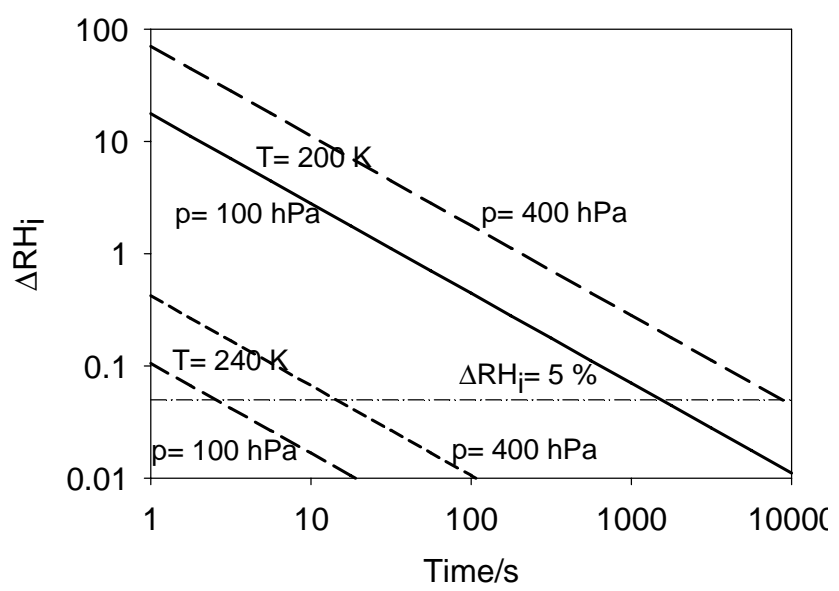

Fig. A4. Relative humidity increase over ice due to engine water vapor emissions versus time for four combinations of ambient pressure and temperature. The $5 \%$ change is high-lighted by a horizontal line

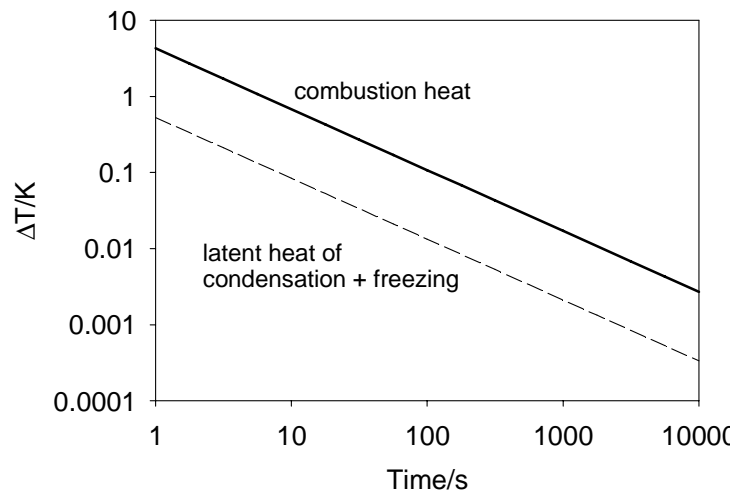

Fig. A5. Temperature increases due to combustion-heat and latentheat releases versus time.

$200 \mathrm{~K}$. For higher temperatures, near $240 \mathrm{~K}$, the emitted water vapor is important only for shorter ages, up to about 1 min.

The temperature increase $\Delta T$ due to combustion heat release is $\Delta T=Q_{\text {fuel }}(1-\eta) /\left(c_{p} N_{\text {dil }}(t)\right)$, see Fig. A5. For comparison, we note that a vertical potential temperature gradient of typically $0.003 \mathrm{~K} \mathrm{~m}^{-1}$ in the troposphere implies $0.3 \mathrm{~K}$ warming for $100 \mathrm{~m}$ altitude increase. The combustion induced temperature increases stay below $0.3 \mathrm{~K}$ after about 1 min age. The warming by latent heat release by condensing all emitted water would be about 10 times smaller because the latent heat is so much smaller than the combustion heat.

Typical ice water contents in cirrus and contrails are

$\mathrm{IWC} /\left(\mathrm{mg} \mathrm{m}^{-3}\right)=\exp \left(6.97+0.103 T /{ }^{\circ} \mathrm{C}\right)$

(Schumann, 2002; Schiller et al., 2008). If all this water gets sublimated, the resultant temperature change remains below $0.1 \mathrm{~K}$ in cold air, for $T<240 \mathrm{~K}$.

\section{A7 Advection near the Poles}

To avoid singularities at the Poles, for large latitude magnitudes $|y|>80^{\circ}$, we invert the longitude (E) and latitude (N) coordinates $x_{n}$ and $y_{n}$ at the previous time step $t_{n}$ first into Cartesian coordinates $X, Y$ (in the directions of $90^{\circ}$ and $180^{\circ}$ from the Pole), then compute advection during the time step $\Delta t=t_{n+1}-t_{n}$ towards new Cartesian coordinates $\tilde{X}, \tilde{Y}$ with the horizontal longitudinal/latitudinal velocity components $u_{n}, v_{n}$ at $\left(x_{n}, y_{n}, p_{n}, t_{n}\right)$ in degree $\mathrm{s}^{-1}$, and then return the new longitude/latitude coordinates to $x_{n+1}, y_{n+1}$ in degree. The angle $\alpha$ measures the negative longitude relative to the $X$-axis-direction. The variable $s$ equals $s=1$ at the North Pole and $s=-1$ at the South Pole.

$$
\begin{aligned}
& S_{x}=\sin \left(x_{n} \pi / 180^{\circ}\right), C_{x}=\cos \left(x_{n} \pi / 180^{\circ}\right), \\
& X=S_{x}\left(90^{\circ}-\left|y_{n}\right|\right), Y=-s C_{x}\left(90^{\circ}-\left|y_{n}\right|\right), \\
& C_{y}=\cos \left(y_{n} \pi / 180^{\circ}\right), \\
& U_{n}=C_{y} u_{n} C_{x}-s v_{n} S_{x}, V_{n}=s C_{y} u_{n} S_{x}+v_{n} C_{x}, \\
& \tilde{X}=X+\Delta t U_{n}, \tilde{Y}=Y+\Delta t V_{n} .
\end{aligned}
$$

These equations apply for the Runge-Kutta predictor step. For the corrector step, $\tilde{X}, \tilde{Y}$ are computed using $U_{n+1}, V_{n+1}$ derived similarly using $u_{n+1}, v_{n+1}$ at $x_{n+1}, y_{n+1}, p_{n+1}, t_{n+1}$ : $\tilde{X}=X+(1 / 2) \Delta t\left[U_{n}+U_{n+1}\right], \quad \tilde{Y}=Y+(1 / 2) \Delta t\left[V_{n}+\right.$ $\left.V_{n+1}\right]$

$$
\begin{aligned}
& \text { and then } \\
& Z=\tilde{X}^{2}+\tilde{Y}^{2}, \alpha=\operatorname{atan}(\tilde{Y}, \tilde{X}) 180^{\circ} / \pi, \\
& x_{n+1}=90^{\circ}+s \alpha, y_{n+1}=s\left[90^{\circ}-Z^{1 / 2}\right] .
\end{aligned}
$$

After call of this routine, one has to make sure (by adding or subtracting $360^{\circ}$ ) that consecutive way points are consecutive in longitude with increments $<180^{\circ}$.

\section{A8 Flight and contrail segments passing the date line}

Flights across the Pacific and some flights near the Poles pass the date line at $\pm 180^{\circ}$ once or several times. On input, flight way points are given consecutively with small longitude increments (we require increment less than $300^{\circ}$, which should be sufficient even close to the Poles). However, flights passing the date line, with some longitude values exceeding $180^{\circ}$ in magnitude, require special attention.

For contrail analysis, all segment coordinates are left unchanged from the input. This allows for straightforward computation of segment length and direction values (the latter is needed for computing the horizontal velocity component $V_{n}$ normal to the segment). Only for interpolation in NWP data, we shift longitudes by $\pm 360^{\circ}$ into the inner range $-180^{\circ}<x \leq 180^{\circ}$.

However, for plots and related analysis, one needs to map the flight paths and contrail segment coordinates to the inner range. This is done (in module TESTD) by inserting two extra points into a segment whenever passing the date line, one with $x=180^{\circ}$, the other with $x=-180^{\circ}$, where the sequence depends on the flight direction. All other contrail properties (except segment length) are interpolated linearly between the original endpoints to the new end points. Special 


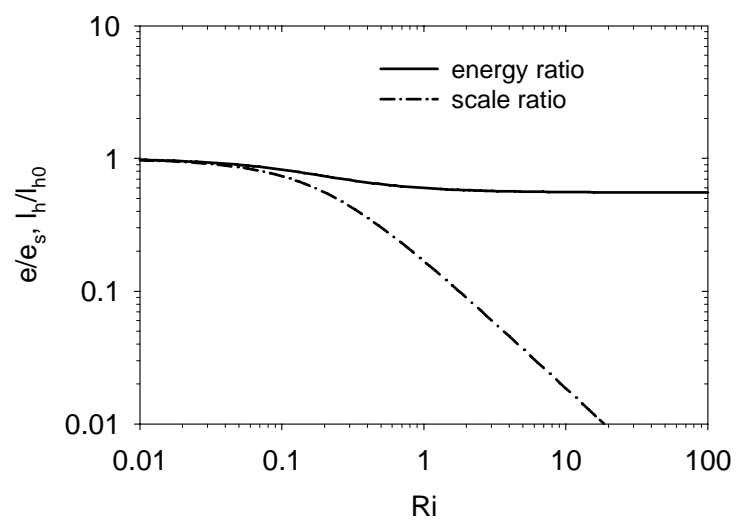

Fig. A6. Normalized subgrid-scale energy $e$ and mixing lengthscale for heat $\ell_{h}$ versus Richardson number, $e_{\mathrm{S}}=e(R i=0), \ell_{h 0}=$ $\ell_{h}(R i=0)$.

care was needed with input coordinates $|x|>540^{\circ}$. Such rare segments crossing this secondary date line are eliminated from further analysis (in module LIMITX).

\section{A9 Subgrid-scale vertical velocity variance}

The kinetic energy of mesoscale subgrid-scale turbulent motions $e$ per unit mass, and its vertical variance part $w_{\text {meso }}^{\prime}$ as needed in Eq. (55), are computed following Schumann (1991):

$e=\ell_{\epsilon}\left(\ell_{m} S_{T}^{2}-\ell_{h} N_{\mathrm{BV}}^{2}\right)$

$w_{\mathrm{SGS}}^{\prime 2} / e=(2 / 3)\left[\ell_{h} /\left(c_{h} \ell\right)\right]^{2}$,

with

$$
\begin{gathered}
\ell_{h}=c_{h} \ell e /\left(e+0.3 \ell^{2} N_{\mathrm{BV}}^{2}\right), \\
\ell_{\epsilon}=\ell / c_{\epsilon}, \ell_{m}=c_{m} \ell .
\end{gathered}
$$

Most of the model coefficients are consequences of an inertial range spectrum assumed at subgrid scales (Schumann, 1991): $c_{\epsilon}=0.845, c_{m}=0.0856, c_{h}=0.204$. The NWP grid scale is set to $\ell=700 \mathrm{~m}$. A larger $\ell$ implies a smaller $E_{\text {meso }}$ in Eq. (55). Hence, this is essentially not an additional free parameter in this application.

Because of the dependence of $\ell_{h}$ on $e$, these equations are quadratic in $e$. The solution is $e=b+\sqrt{b^{2}+c}$, with $b=\alpha_{\mathrm{S}} S_{T}^{2}-\alpha_{N} N_{\mathrm{BV}}^{2}, c=\alpha_{c} S_{T}^{2} N_{\mathrm{BV}}^{2}, \alpha_{\mathrm{S}}=\ell_{\epsilon} \ell_{m} / 2, \alpha_{N}=$ $\left(0.3 \ell+\ell_{\epsilon} c_{h}\right) \ell / 2, \alpha_{c}=0.3 \ell^{2} \ell_{\epsilon} \ell_{m}$. The solution $e$ is nonnegative for any positive or negative stratification.

Normalized results are functions of the Richardson number $R i$. The energy $e$ stays rather large, but the turbulence becomes highly anisotropic with decreasing $w_{\mathrm{SGS}}^{\prime 2}$ for growing $R i$. The ratio of vertical to total kinetic SGS energy $w_{\mathrm{SGS}}^{\prime 2} / e$ is $(2 / 3)$ for locally isotropic turbulence at weak stratification and tends to zero for $\ell^{2} N_{\mathrm{BV}}^{2} / e \rightarrow 0$, see Fig. A6.

\begin{tabular}{|c|c|c|}
\hline Parameter & Explanation & Unit \\
\hline$A$ & contrail cross-section area & $\mathrm{m}^{2}$ \\
\hline$B$ & contrail breadth & $\mathrm{m}$ \\
\hline$c$ & concentration & $\mathrm{kg} \mathrm{m}^{-3}$ \\
\hline$c_{p}$ & specific heat capacity of air & $\mathrm{J}(\mathrm{kg} \mathrm{K})^{-1}$ \\
\hline$C$ & fractional cloud cover & 1 \\
\hline$C_{0}$ & mass of a plume species per length & $\mathrm{kg} \mathrm{m}^{-1}$ \\
\hline$d$ & particle diameter & $\mathrm{m}$ \\
\hline$D$ & contrail depth & $\mathrm{m}$ \\
\hline$D_{\text {eff }}$ & effective contrail depth $A / B$ & $\mathrm{~m}$ \\
\hline$D_{\mathrm{H}}$ & horizontal diffusivity & $\mathrm{m}^{2} \mathrm{~s}^{-1}$ \\
\hline$D_{\mathrm{V}}$ & vertical diffusivity & $\mathrm{m}^{2} \mathrm{~s}^{-1}$ \\
\hline$D_{\mathrm{S}}$ & off-diagonal "shear" diffusivity & $\mathrm{m}^{2} \mathrm{~s}^{-1}$ \\
\hline$e^{5}$ & kinetic energy of turbulent subgrid-scale motions & $\mathrm{m}^{2} \mathrm{~s}^{-2}$ \\
\hline$E_{\mathrm{SGS}}$ & kinetic energy of all subgrid-scale motions & $\mathrm{m}^{2} \mathrm{~s}^{-2}$ \\
\hline EI & emission index, mass or number per fuel mass & $\mathrm{kg} \mathrm{kg}^{-1}, \mathrm{~kg}^{-1}$ \\
\hline$f_{\text {surv }}$ & fraction of particles surviving the wake vortex phase & 1 \\
\hline$g$ & gravity & $\mathrm{m} \mathrm{s}^{-2}$ \\
\hline$G$ & mixing line gradient & $\mathrm{Pa} \mathrm{K}^{-1}$ \\
\hline I & ice mass mixing ratio & $\mathrm{kg} \mathrm{kg}^{-1}$ \\
\hline IWC & ice water concentration & $\mathrm{kg} \mathrm{m}^{-3}$ \\
\hline IWP & ice water path & $\mathrm{kg} \mathrm{m}^{-2}$ \\
\hline$\ell$ & NWP subgrid length scale & $\mathrm{m}$ \\
\hline$L$ & horizontal segment length & $\mathrm{m}$ \\
\hline$L_{\mathrm{S}}$ & latent heat of sublimation & $\mathrm{J} \mathrm{kg}^{-1}$ \\
\hline$m_{F}$ & fuel consumption per flight distance & $\mathrm{kg} \mathrm{m}^{-1}$ \\
\hline$M$ & plume mass per contrail length & $\mathrm{kg} \mathrm{m}^{-1}$ \\
\hline$M_{\mathrm{S}}$ & molar mass of species $\mathrm{s}\left(\mathrm{air}, \mathrm{H}_{2} \mathrm{O}\right)$ & $\mathrm{kg} \mathrm{mol}^{-1}$ \\
\hline$M_{\mathrm{a}}$ & aircraft mass & $\mathrm{kg}$ \\
\hline$n$ & number of ice particles per volume & $\mathrm{m}^{-3}$ \\
\hline$N$ & total ice number concentration per contrail length & $\mathrm{m}^{-1}$ \\
\hline$N_{\mathrm{BV}}$ & Brunt-Vaisaila frequency & $s^{-1}$ \\
\hline$N_{W}$ & number of waypoints & 1 \\
\hline$N_{\text {dil }}$ & ratio between contrail mass and fuel flow per length & 1 \\
\hline OLR & outgoing longwave radiation & $\mathrm{W} \mathrm{m}^{-2}$ \\
\hline$p$ & pressure & $\mathrm{Pa}$ \\
\hline$p_{\text {liq }}$ & liquid saturation pressure & $\mathrm{Pa}$ \\
\hline$p_{\text {ice }}$ & ice saturation pressure & $\mathrm{Pa}$ \\
\hline$q$ & absolute humidity (mass fraction of water vapor in air) & $\mathrm{kg} \mathrm{kg}^{-1}$ \\
\hline$q_{\mathrm{s}}$ & ice saturation humidity & $\mathrm{kg} \mathrm{kg}^{-1}$ \\
\hline$Q_{\text {ext }}$ & solar radiation extinction efficiency & 1 \\
\hline
\end{tabular}

Table A1. List of symbols.

For $S_{T}=0.002 \mathrm{~s}^{-1}, N_{\mathrm{BV}}=0.02 \mathrm{~s}^{-1}$, the result is $e \approx$ $0.11 \mathrm{~m}^{2} \mathrm{~s}^{-2}$, which is within the range of measured values (Schumann et al., 1995). For $R i \approx 10$, the modeled ratio is $w^{\prime 2} / e \approx 0.02$, while the measurements show a ratio of about 0.15 . Therefore, we enhance $w_{\text {SGS }}^{\prime 2}$ by adding the variance of mean vertical motions $\bar{w}$ at grid scales, see Eq. (54).

\section{A10 Particle number integration}

For the number of particles per contrail length, we need to integrate an ordinary differential equation of the form

$N^{\prime}=-\mathrm{AN}^{2}-\mathrm{BN}, N(0)=N_{0}$.

The analytical solution of this equation is:

$N(t)=\frac{\mathrm{BN}_{0} e^{-B t}}{B+\mathrm{AN}_{0}\left(1-e^{-B t}\right)}$, for $B t>0$.

The solution simplifies to

$N(t)=\frac{N_{0}}{1+\mathrm{AN}_{0}}$, for $B t \ll 1$, 
Table A1. Continued.

\begin{tabular}{|c|c|c|}
\hline Parameter & Explanation & Unit \\
\hline$Q_{\text {fuel }}$ & heat of fuel combustion & $\mathrm{MJ} \mathrm{kg}^{-1}$ \\
\hline$r$ & volume mean particle radius & $\mathrm{m}$ \\
\hline$r_{\text {eff }}$ & optically effective particle radius & $\mathrm{m}$ \\
\hline$R_{0}, R_{1}$ & gas constants of air and water vapor & $\mathrm{J}(\mathrm{kg} \mathrm{K})^{-1}$ \\
\hline RHi & relative humidity over ice & 1 \\
\hline$R i$ & Richardson number $N_{\mathrm{BV}}^{2} / S_{T}^{2}$ & 1 \\
\hline $\mathrm{RF}$ & radiative forcing & $\mathrm{W} \mathrm{m}^{-2}$ \\
\hline RSR & reflected shortwave radiation & $\mathrm{W} \mathrm{m}^{-2}$ \\
\hline$s_{\mathrm{a}}$ & aircraft wing span & $\mathrm{m}$ \\
\hline SDR & solar direct radiation & $\mathrm{W} \mathrm{m}^{-2}$ \\
\hline$S_{0}$ & solar constant & $\mathrm{W} \mathrm{m}^{-2}$ \\
\hline$S_{i}$ & flight segment $\left(W_{i}, W_{i}+1\right)$ & \\
\hline$S, S_{T}$ & shear perpendicular to plume, total shear & $\mathrm{s}^{-1}$ \\
\hline$t$ & time or age & $\mathrm{s}$ \\
\hline$T$ & absolute temperature & K \\
\hline$T_{\mathrm{LC}}$ & threshold temperature & K \\
\hline$T_{\mathrm{LM}}$ & maximum threshold temperature & $\mathrm{K}$ \\
\hline$U$ & air speed in $\mathrm{x}$-direction & $\mathrm{m} \mathrm{s}^{-1}$ \\
\hline$U$ & relative humidity of liquid saturation & 1 \\
\hline$V$ & air speed in y-direction & $\mathrm{m} \mathrm{s}^{-1}$ \\
\hline$V_{\mathrm{a}}$ & aircraft true airspeed & $\mathrm{m} \mathrm{s}^{-1}$ \\
\hline$V_{T}$ & particle terminal fall velocity & $\mathrm{m} \mathrm{s}^{-1}$ \\
\hline$w$ & vertical velocity & $\mathrm{m} \mathrm{s}^{-1}$ \\
\hline$w_{N}^{\prime}$ & stratified turbulence velocity scale & $\mathrm{m} \mathrm{s}^{-1}$ \\
\hline$W_{i}$ & waypoint $\left(x_{i}, y_{i}, z_{i}, t_{i}\right), i=1,2, \ldots, N_{W}$ & $\circ, \circ, \mathrm{m}, \mathrm{s}$ \\
\hline$x$ & longitude & $\circ \quad$ \\
\hline$X$ & vector of variables $(x, y, p, \sigma, I, N)$ & \\
\hline$y$ & latitude & $\circ$ \\
\hline$z$ & altitude above sea level & $\mathrm{m}$ \\
\hline$\epsilon$ & kinetic energy dissipation rate & $\mathrm{m}^{2} \mathrm{~s}^{-3}$ \\
\hline$\Delta t$ & time step & $\mathrm{s}$ \\
\hline$\Delta z_{w}$ & downwash distance & $\mathrm{m}$ \\
\hline$\eta$ & overall propulsion efficiency & 1 \\
\hline$\Gamma$ & wake vortex circulation & $\mathrm{m}^{2} \mathrm{~s}^{-1}$ \\
\hline$\mu$ & cosine of solar zenith angle & 1 \\
\hline$\rho, \rho_{\text {ice }}$ & air, bulk ice density & $\mathrm{kg} \mathrm{m}^{-3}$ \\
\hline$\omega$ & pressure change rate & $\mathrm{Pa} \mathrm{s}^{-1}$ \\
\hline$\sigma$ & covariance matrix & $\mathrm{m}^{2}$ \\
\hline$\sigma_{y y}, \sigma_{z z}, \sigma_{y z}$ & elements of $\sigma$ & $\mathrm{m}^{2}$ \\
\hline$\tau$ & optical depth at $550 \mathrm{~nm}$ & 1 \\
\hline$\tau_{d i l}$ & time scale of dilution & $\mathrm{s}$ \\
\hline$\theta$ & solar zenith angle & $\circ$ \\
\hline$\Theta$ & potential temperature & $\mathrm{K}$ \\
\hline
\end{tabular}

$N(t)=N_{0} e^{-B t}$, for $\mathrm{AN}_{0} \ll B$.

\section{A11 One-dimensional Gaussian integral}

The function $f_{\tau}(y)$, Eq. (63), follows from

$f_{\tau}(y)=(B / A) I(y)$, with

$I(y)=\int_{-\infty}^{\infty} \exp \left[-(1 / 2) \mathbf{x}^{T} \sigma^{-1} \mathbf{x}\right] d z$.

This integral can be evaluated (Abramowitz and Stegun,

1964, 7.4.2, 7.1.2 and 7.1.9),

$$
\begin{aligned}
& I(y)=\int_{-\infty}^{\infty} \exp \left[-\left(a z^{2}+2 b z+c\right)\right] d z \\
& =(\pi / a)^{1 / 2} \exp \left[\left(b^{2}-a c\right) / a\right] \\
& \text { where } \\
& a=(1 / 2) \sigma_{y y} / \operatorname{det} \sigma, b=(1 / 2) \sigma_{y z} / \operatorname{det} \sigma,
\end{aligned}
$$

Table A2. Abbreviations.

\begin{tabular}{ll}
\hline Acronym & Explanation \\
\hline ACCRI & FAA-project "Aviation Climate Change Research Initiative" \\
BADA & Base of Aircraft Date from EUROCONTROL \\
CATS & DLR-project "Climate-compatible Air Transport System" \\
CI & cirrus \\
CO & contrail \\
COCI & contrail cirrus \\
CoCiP & Contrail Cirrus Prediction tool \\
CONCERT & CONtrail and Cirrus ExpeRimenT \\
COSMO-DE & Consortium for Small Scale Modelling - \\
& Deutschland, NWP model of DWD \\
DFS & Deutsche Flugsicherung \\
DLR & Deutsches Zentrum für Luft- und Raumfahrt \\
DWD & Deutscher Wetterdienst \\
ECMWF & European Centre for Medium Range Weather Forecasts \\
EU & European Union \\
EUROCONTROL & European Organization for the Safety of Air Navigation \\
FAA & Federal Aviation Administration \\
ICAO & International Civil Aviation Organization \\
IFS & Integrated Forecasting System \\
ISSR & ice supersaturated region \\
LES & large-eddy simulation \\
LW & longwave \\
NWP & numerical weather prediction \\
OAG & Official Airline Guide \\
OLR & outgoing longwave radiation \\
rms & root-mean-square \\
RSR & reflected shortwave radiation \\
REACT-4C & EU project "Reducing Emissions from Aviation by \\
SAC & Changing Trajectories for the benefit of Climate" \\
SGS & Schmidt-Appleman criterion \\
SW & subgrid scale \\
TOA & shortwave \\
US, USA & top of atmosphere \\
2d, 3d & United States, .. of America \\
\hline & two-dimensional, three-dimensional \\
\hline &
\end{tabular}

and $c=(1 / 2) \sigma_{z z} / \operatorname{det} \sigma$.

With Eqs. $(3,8)$,

$A=2 \pi(\operatorname{det} \sigma)^{1 / 2}$ and $\sigma_{y y}=B^{2} / 8$,

this results into Eq. (63),

$f_{\tau}(y)=(4 / \pi)^{1 / 2}(A / B) \exp \left[-(1 / 2) y^{2} / \sigma_{y y}\right]$

independent of $\sigma_{y z}$.

\section{A12 Contrail segment contribution to optical depth in the cloud-mask}

For given Cartesian segment end-points $\left(x_{1}, y_{1}\right),\left(x_{2}, y_{2}\right)$, we compute the contribution of the segment to the optical depth $\tau_{0,0}$ at the pixel position, e.g. at $\left(x_{0}, y_{0}\right)$, using linear geometry, see Fig. A7, as follows. First, a parameter $w$ is computed,

$$
\begin{aligned}
& \Delta x_{0}=x_{2}-x_{1}, \Delta y_{0}=y_{2}-y_{1} \\
& \operatorname{det}=\left(\Delta x_{0}\right)^{2}+\left(\Delta y_{0}\right)^{2} \\
& \Delta x_{1}=x_{0}-x_{1}, \Delta y_{1}=y_{0}-y_{1} \\
& w=\left(\Delta x_{1} \Delta x_{0}+\Delta y_{1} \Delta y_{0}\right) / \text { det, }
\end{aligned}
$$

with det> 0 if the normal from $\left(x_{0}, y_{0}\right)$ crosses the segment line. If the cross-point $\left(x_{\mathrm{s}}, y_{\mathrm{s}}\right)$ is within the segment line, i.e. for $|w-1 / 2|<1 / 2$, we compute the distance $s$ to the pixel point and with this the contribution to optical depth.

$$
\begin{aligned}
& x_{\mathrm{s}}=x_{1}+w\left(x_{2}-x_{1}\right), y_{\mathrm{s}}=y_{1}+w\left(y_{2}-y_{1}\right) \\
& s^{2}=\left(x_{0}-x_{\mathrm{s}}\right)^{2}+\left(y_{0}-y_{\mathrm{s}}\right)^{2} \\
& B=w B_{2}+(1-w) B_{1}
\end{aligned}
$$




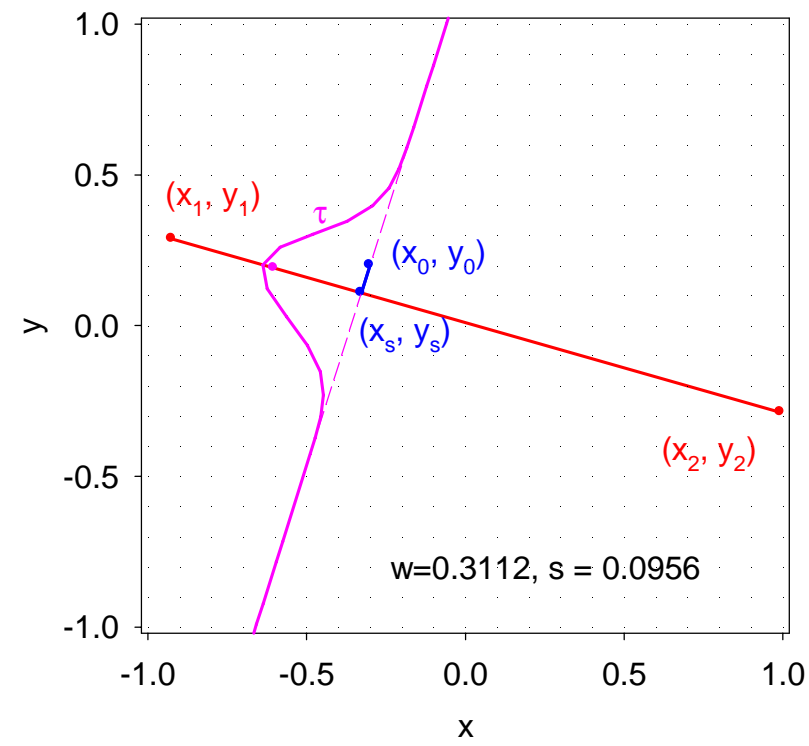

Fig. A7. Example of a contrail-segement between end-points $\left(x_{1}, y_{1}\right)$ and $\left(x_{2}, y_{2}\right)$ (red) with closest connection (blue) from a point $\left(x_{0}, y_{0}\right)$ in the cloud mask to $\left(x_{s}, y_{s}\right)$ on the contrail centerline, and contribution to optical depth $\tau$ (see Sects. 2.15 and A12).

$$
\begin{aligned}
& \delta=s^{2} /\left(B^{2} / 8\right) \\
& \gamma=(4 / \pi)^{1 / 2} \exp [-(1 / 2) \delta] \\
& \tau=w \tau_{2}+(1-w) \tau_{1} \\
& \tau_{0,0}:=\tau_{0,0}+\gamma \tau
\end{aligned}
$$

Here, $B_{m}$ and $\tau_{m}$ are the contrail width and optical depth at the segment end points, $m=1,2$.

Acknowledgements. Important contributions by several colleagues are gratefully acknowledged. Among these, Frank Holzäpfel contributed the non-dimensional parameterization of the wake vortex sinking distance and data shown in Fig. 3. Simon Unterstrasser contributed numerical output from his model for comparisons. Kaspar Graf helped with the emission data, a large number of tests, plots, and comparisons to satellite data (to be described elsewhere). Andreas Dörnbrack helped with access to the ECMWF data. Klaus Gierens, Bernd Kärcher, and Simon Unterstrasser provided valuable comments on a draft version. Aircraft data were provided within the BADA data set by EUROCONTROL. Air traffic data were provided to us by FAA and EUROCONTROL, partly within the project ACCRI. This work is part of the DLR project CATS and the EU project REACT4C.

Edited by: O. Boucher

\section{References}

Abramowitz, M. and Stegun, I. A.: Handbook of Mathematical Functions, Dover Publisher, New York, 1046 pp., 1964.

Adelfang, S. I.: On the relations between wind shears over various altitude intervals, J. Appl. Meteor., 10, 156-159, 1971.
Appleman, H.: The formation of exhaust contrails by jet aircraft, B Am. Meteor. Soc., 34, 14-20, 1953.

Atlas, D. and Wang, Z.: Contrails of small and very large optical depth, J. Atmos. Sci., 67, 3065-3073, doi:10.1175/2010JAS3403.1, 2010.

Atlas, D., Wang, Z., and Duda, D. P.: Contrails to cirrus - Morphology, microphysics, and radiative properties, J. Appl. Meteor. Climatol., 45, 5-19, 2006.

Bakan, S., Betancor, M., Gayler, V., and Graß1, H.: Contrail frequency over Europe from NOAA-satellite images, Ann. Geophys., 12, 962-968, doi:10.1007/s00585-994-0962-y, 1994.

Baldauf, M., Seifert, A., Förstner, J., Majewski, D., Raschendorfer, M., and Reinhardt, T.: Operational convective-scale numerical weather prediction with the COSMO model: Description and sensitivities, Mon. Wea. Rev., 139, 3887-3905, doi:10.1175/MWR-D-10-05013.1, 2011.

Birner, T., Dörnbrack, A., and Schumann, U.: How sharp is the tropopause at midlatitudes?, Geophys. Res. Lett., 29, 45-1-454, doi:10.1029/2002g1015142, 2002.

Blanco-Muriel, M., Alarcón-Padilla, D. C., López-Moratella, T., and Lara-Coira, M.: Computing the solar vector, Solar Energy, 70, 431-441, 2001.

Brown, R. C., Miake-Lye, R. C., Anderson, M. R., and Kolb, C. E.: Aircraft sulphur emissions and the formation of visible contrails, Geophys. Res. Lett., 12, 385-388, doi:10.1029/97GL00107, 1997.

Burkhardt, U. and Kärcher, B.: Global radiative forcing from contrail cirrus, Nature Clim. Change, 1, 54-58, doi:10.1038/NCLIMATE1068, 2011.

Burkhardt, U., Kärcher, B., and Schumann, U.: Global modelling of the contrail and contrail cirrus climate impact, B. Am. Meteor. Soc., 91, 479-484, doi:10.1175/2009BAMS2656.1, 2010.

Busen, R. and Schumann, U.: Visible contrail formation from fuels with different sulfur contents, Geophys. Res. Lett., 22, 13571360, doi:10.1029/95GL01312, 1995.

Cariolle, D., Caro, D., Paoli, R., Hauglustaine, D. A., Cuenot, B., Cozic, A., and Paugam, R.: Parameterization of plume chemistry into large-scale atmospheric models: Application to aircraft $\mathrm{NO}_{\mathrm{x}}$ emissions, J. Geophys. Res., 114, D19302, doi:10.1029/2009JD011873, 2009.

Chlond, A.: Large eddy simulations of contrails, J. Atmos. Sci., 55, 796-819, 1998.

Clayson, C. A. and Kantha, L.: On turbulence and mixing in the free atmosphere inferred from high-resolution soundings, J. Atmos. Oceanic Technol., 25, 833-852, 2008.

Danielsen, E. F.: Trajectories: Isobaric, isentropic and actual, J. Meteorol., 18, 479-486, 1961.

Danilin, M., Ebel, A., Elbern, H., and Petry, H.: Evolution of the concentrations of trace species in an aircraft plume: Trajectory study, J. Geophys. Res., 99, 18951-18972, doi:10.1029/94JD01820, 1994.

deBruin, A. and Kannemans, H.: Analysis of NLR Cessna Citation flight test data for flight test-1 in AWIATOR project, Tech. rep., NLR, Techn. Report AW-NLR-113-010, 2004.

Delisi, D. P. and Robins, R.: Short-scale instabilities in trailing wake vortices in a stratified fluid, AIAA J., 38, 1916-1923, 2000.

Detwiler, A. and Pratt, R.: Clear-air seeding: Opportunities and strategies, J. Wea. Mod., 16, 46-60, 1984. 
Dewan, E. M.: Stratospheric wave spectra resembling turbulence, Science, 204, 832-835, doi:10.1126/science.204.4395.832, 1979.

Dobbie, S. and Jonas, P.: Radiative influences on the structure and lifetime of cirrus clouds, Q. J. Roy. Meteor. Soc., 127, 26632682, 2001.

Dörnbrack, A. and Dürbeck, T.: Turbulent dispersion of aircraft exhausts in regions of breaking gravity waves, Atmos. Environ., 32, 3105-3112, 1998.

Duda, D., Minnis, P., and Nguyen, L.: Estimates of cloud radiative forcing in contrail clusters using GOES imagery, J. Geophys. Res., 106, 4927-4937, 2001.

Duda, D., Minnis, P., Nyuyen, L., and Palikonda, R.: A case study of the development of contrail clusters over the Great Lakes, J. Atmos. Sci., 61, 1132-1146, 2004.

Duda, D. P., Palikonda, R., and Minnis, P.: Relating observations of contrail persistence to numerical weather analysis output, Atmos. Chem. Phys., 9, 1357-1364, doi:10.5194/acp-9-1357-2009, 2009.

Dürbeck, T. and Gerz, T.: Large-eddy simulation of aircraft exhaust plumes in the free atmosphere: Effective diffusivities and cross-sections, Geophys. Res. Lett., 22, 3203-3206, doi:10.1029/95GL03021, 1995.

Dürbeck, T. and Gerz, T.: Dispersion of aircraft exhausts in the free atmosphere, J. Geophys. Res., 101, 26007-26015, 1996.

EUROCONTROL: Aircraft Performance Summary Tables for the Base of Aircraft Date (BADA), Revision 3.7, Tech. rep., European Organisation for the Safety of Air Navigation, 2009.

Eyers, C. J., Addleton, D., Atkinson, K., Broomhead, M. J., Christou, R., Elliff, T., Falk, R., Gee, I., Lee, D. S., Marizy, C., Michot, S., Middel, J., Newton, P., Norman, P., Plohr, M., Raper, D., and Stanciou, N.: AERO2k Global Aviation Emissions Inventories for 2002 and 2025, Tech. rep., QinetiQ for European Commission under Contract No. G4RD-CT-2000-00382, http: //www.cate.mmu.ac.uk/aero2k.asp, 2004.

Fahey, D., Schumann, U., Ackerman, S., Artaxo, P., Boucher, O., Danilin, M. Y., Kärcher, B., Minnis, P., Nakajima, T., and Toon, O. B.: Aviation-produced aerosols and cloudiness, in: Aviation and the Global Atmosphere. A Special Report of IPCC Working Groups I and III, edited by: Penner, J. E., Lister, D. H., Griggs, D. J., Dokken, D. J., and McFarland, M., 65120, Cambridge University Press, New York, http://www.ipcc. ch/ipccreports/sres/aviation/index.php?idp=0, 1999.

Febvre, G., Gayet, J.-F., Minikin, A., Schlager, H., Shcherbakov, V., Jourdan, O., Busen, R., Fiebig, M., Kärcher, B., and Schumann, U.: On optical and microphysical characteristics of contrails and cirrus, J. Geophys. Res., 114, D02204, doi:10.1029/2008JD010184, 2009.

Ferrone, A.: Aviation and climate change in Europe: from regional climate modelling to policy-options, Ph.D. thesis, Université Catholique de Louvain, 2011.

Fueglistaler, S., Legras, B., Beljaars, A., Morcrette, J.-J., Simmons, A., Tompkins, A. M., and Uppala, S.: The diabatic heat budget of the upper troposphere and lower/mid stratosphere in ECMWF reanalyses, Q. J. Roy. Meteor. Soc., 135, 21-37, doi:10.1002/qj.361, 2010.

Frehlich, R. and Sharman, R.: Climatology of velocity and temperature turbulence statistics determined from rawinsonde and ACARS/AMDAR data, J. Appl. Meteor. Climatol., 49, 1149-
1169, doi:10.1175/2010JAMC2196, 2010.

Freudenthaler, V., Homburg, F., and Jäger, H.: Contrail observations by ground-based scanning lidar: Cross-sectional growth, Geophys. Res. Lett., 22, 3501-3504, doi:10.1029/95GL03549, 1995.

Garber, D. P., Minnis, P., and Costulis, P. K.: A commercial flight track database for upper tropospheric aircraft emission studies over the USA and southern Canada, Meteor. Z., 14, 445-452, 2005.

Gayet, J.-F., Febvre, G., Brogniez, G., Chepfer, H., Renger, W., and Wendling, P.: Microphysical and optical properties of cirrus and contrails, J. Atmos. Sci., 53, 126-138, 1996.

Gerz, T. and Ehret, T.: Wake dynamics and exhaust distribution behind cruising aircraft, in: The Characterization and Modification of Wakes from Lifting Vehicles in Fluids, 35.1-35.8, AGARD CP 584, 1996.

Gerz, T., Dürbeck, T., and Konopka, P.: Transport and effective diffusion of aircraft emissions, J. Geophys. Res., 103, doi:10.1029/98JD02282, 1998.

Gierens, K.: Numerical simulations of persistent contrails, J. Atmos. Sci., 53, 3333-3348, 1996.

Gierens, K. and Bretl, S.: Analytical treatment of ice sublimation and test of sublimation parameterisations in two-moment ice microphysics models, Atmos. Chem. Phys., 9, 7481-7490, doi:10.5194/acp-9-7481-2009, 2009.

Gierens, K. and Jensen, E.: A numerical study of the contrail-tocirrus transition, Geophys. Res. Lett., 25, 4341-4344, 1998.

Gierens, K. and Spichtinger, P.: On the size distribution of icesupersaturated regions in the upper troposphere and lowermost stratosphere, Ann. Geophys., 18, 499-504, doi:10.1007/s00585000-0499-7, 2000.

Gierens, K., Schumann, U., Helten, M., Smit, H., and Marenco, A.: A distribution law for relative humidity in the upper troposphere and lower stratosphere derived from three years of MOZAIC measurements, Ann. Geophys., 17, 1218-1226, doi:10.1007/s00585-999-1218-7, 1999.

Gierens, K., Kärcher, B., Mannstein, H., and Mayer, B.: Aerodynamic contrails: Phenomenology and flow physics, J. Atmos Sci., 66, 217-226, doi:10.1175/2008JAS2767.1, 2009.

Gierens, K. M.: The influence of radiation on the diffusional growth of ice crystals, Beitr. Phys. Atmos., 67, 181-193, 1994.

Green, J. E.: Greener by design - the technology challenge, Aeron. J., 106, 57-113, 2002.

Gultepe, I. and Starr, D. O.: Dynamical structure and turbulence in cirrus clouds: Aircraft observations during FIRE, J. Atmos. Sci., 52, 4159-4182, 1995.

Hansen, J. E. and Travis, L. D.: Light scattering in planetary atmospheres, Space Sci. Rev., 16, 527-610, 1974.

Haywood, J. M., Allan, R. P., Bornemann, J., Forster, P. M., Francis, P. N., Milton, S., Rädel, G., Rap, A., Shine, K. P., and Thorpe, R.: A case study of the radiative forcing of persistent contrails evolving into contrail-induced cirrus, J. Geophys. Res., 114, D24201, doi:10.1029/2009JD012650, 2009.

Hennemann, I.: Deformation und Zerfall von Flugzeugwirbelschleppen in turbulenter und stabil geschichteter Atmosphäre, Tech. rep., Deutsches Zentrum für Luft- und Raumfahrt, Forschungsbericht 2010-21, 2010.

Heymsfield, A. J., Lawson, R. P., and Sachse, G. W.: Growth of ice crystals in a precipitating contrail, Geophys. Res. Lett., 25, 1335-1338, 1998. 
Holzäpfel, F.: Probabilistic two-phase wake vortex decay and transport model, J. Aircraft, 40, 323-331, 2003.

Holzäpfel, F. and Gerz, T.: Two-dimensional wake vortex physics in the stably stratified atmosphere, Aeros. Sci. Techn., 5, 261-270, 1999.

Holzäpfel, F., Misaka, T., and Hennemann, I.: Wake-vortex topology, circulation, and turbulent exchange processes, in: AIAA Paper 2010-7992, 1-16, 2010.

Houchi, K., Stoffelen, A., Marseille, G. J., and De Kloe, J.: Comparison of wind and wind shear climatologies derived from highresolution radiosondes and the ECMWF model, J. Geophys. Res., 115, D22123, doi:10.1029/2009JD013196, 2010.

Huebsch, W. W. and Lewellen, D. C.: Sensitivity study on contrail evolution, in: 36th AIAA Fluid Dynamics Conference and Exhibit, AIAA 2006-3749, 1-14, 2006.

Hunt, J. C. R.: Diffusion in the stably stratified atmospheric boundary layer, J. Climate Appl. Meteor., 24, 1187-1195, 1985.

Hunt, J. C. R., Stretch, D. D., and Britter, R. E.: Length scales in stably stratified turbulent flows and their use in turbulence models, in: Stably Stratified Flows and Dense Gas Dispersion, edited by: Puttock, J. S., 285-321, Clarendon, 1988.

ICAO: Manual of the ICAO Standard Atmosphere, Tech. rep., ICAO Document No. 7488, 2nd Edition, 1964.

Immler, F., Treffeisen, R., Engelbart, D., Krüger, K., and Schrems, O.: Cirrus, contrails, and ice supersaturated regions in high pressure systems at northern mid latitudes, Atmos. Chem. Phys., 8, 1689-1699, doi:10.5194/acp-8-1689-2008, 2008.

IPCC: Climate Change 2007: The Physical Science Basis. Contribution of Working Group I to the Fourth Assessment Report of the Intergovernmental Panel on Climate Change, Cambridge Univ. Press, Cambridge, UK, 996 pp., 2007.

Iwabuchi, H., Yang, P., Liou, K. N., and Minnis, P.: Physical and optical properties of persistent contrails: Climatology and interpretation, J. Geophys. Res., 117, D06215, doi:10.1029/2011JD017020, 2012.

Jensen, E. J., Ackermann, A. S., Stevens, D. E., Toon, O. B., and Minnis, P.: Spreading and growth of contrails in a sheared environment, J. Geophys. Res., 103, 13557-13567, 1998a.

Jensen, E. J., Toon, O. B., Pueschel, R. F., Goodman, J., Sachse, G. W., Anderson, B. E., Chan, K. R., Baumgardner, D., and Miake-Lye, R. C.: Ice crystal nucleation and growth in contrails forming at low ambient temperatures, Geophys. Res. Lett., 25, 1371-1374, doi:10.1029/97GL03592, 1998b.

Jensen, E. J., Pfister, L., and Toon, O. B.: Impact of radiative heating, wind shear, temperature variability, and microphysical processes on the structure and evolution of thin cirrus in the tropical tropopause layer, J. Geophys. Res., 116, D12209, doi:10.1029/2010JD015417, 2011.

Kantha, L. and Hocking, W.: Dissipation rates of turbulence kinetic energy in the free atmosphere: MST radar and radiosondes, J. Atm. Sol. Terr. Phys., 73, 1043-1051, doi:10.1016/j.jastp.2010.11.024, 2011.

Kärcher, B.: A trajectory box model for aircraft exhaust plumes, J. Geophys. Res., 100, 18835-18844, doi:10.1029/95JD01638, 1995.

Kärcher, B.: Physicochemistry of aircraft-generated liquid aerosols, soot, and ice particles: 1. Model description, J. Geophys. Res., 103, 17111-17128, 1998.
Kärcher, B. and Ström, J.: The roles of dynamical variability and aerosols in cirrus cloud formation, Atmos. Chem. Phys., 3, 823 838, doi:10.5194/acp-3-823-2003, 2003.

Kärcher, B. and Yu, F.: Role of aircraft soot emissions in contrail formation, Geophys. Res. Lett., 36, L01804, doi:10.1029/2008GL036649, 2009.

Kärcher, B., Peter, T., Biermann, U. M., and Schumann, U.: The initial composition of jet condensation trails, J. Atmos. Sci., 53, 3066-3083, 1996.

Kärcher, B., Busen, R., Petzold, A., Schröder, F. P., Schumann, U., and Jensen, E. J.: Physicochemistry of aircraft-generated liquid aerosols, soot, and ice particles. 2. Comparison with observations and sensitivity studies, J. Geophys. Res., 103, 1712917147, 1998

Kärcher, B., Burkhardt, U., Unterstrasser, S., and Minnis, P.: Factors controlling contrail cirrus optical depth, Atmos. Chem. Phys., 9, 6229-6254, doi:10.5194/acp-9-6229-2009, 2009a.

Kärcher, B., Mayer, B., Gierens, K., Burkhardt, U., Mannstein, H., and Chatterjee, R.: Aerodynamic contrails: Microphysics and optical properties, J. Atmos. Sci., 66, 227-243, doi:10.1175/2008JAS2768.1, 2009b.

Karol, I. L., Ozolin, Y. E., and Rozanov, E. V.: Box and Gaussian plume models of the exhaust composition evolution of subsonic transport aircraft in- and out of the flight corridor, Ann. Geophys., 15, 88-96, doi:10.1007/s00585-997-0088-0, 1997.

Knollenberg, R.: Measurements of the growth of the ice budget in a persisting contrail, J. Atmos. Sci., 29, 1367-1374, 1972.

Koch, A., Nagel, B., Gollnick, V., Dahlmann, K., Grewe, V., Kärcher, B., and Schumann, U.: Integrated analysis and design environment for a climate compatible air transport system, in: 9th AIAA Aviation Technology, Integration and Operations (ATIO) Conference, pp. AIAA 2009-7050, AIAA, 2009.

Konopka, P.: Analytical Gaussian solutions for anisotropic diffusion in a linear shear flow, J. Non.-Equilib. Thermodyn., 20, 78-91, 1995.

Konrad, T. G. and Howard, J. C.: Multiple contrail streamers observed by radar, J. Appl. Meteor., 13, 563-572, 1974.

Koop, T., Luo, B., Tsias, A., and Peter, T.: Water activity as the determinant for homogeneous ice nucleation in aqueous solutions, Nature, 406, 611-614, 2000.

Kraabøl, A. G., Flatøy, F., and Stordal, F.: Impact of $\mathrm{NO}_{\mathrm{x}}$ emissions from subsonic aircraft: Inclusion of plume processes in a three-dimensional model covering Europe, North America, and the North Atlantic, J. Geophys. Res., 105, 3573-3581, 2000.

Krämer, M., Schiller, C., Afchine, A., Bauer, R., Gensch, I., Mangold, A., Schlicht, S., Spelten, N., Sitnikov, N., Borrmann, S., de Reus, M., and Spichtinger, P.: Ice supersaturations and cirrus cloud crystal numbers, Atmos. Chem. Phys., 9, 3505-3522, doi:10.5194/acp-9-3505-2009, 2009.

Lamquin, N., Gierens, K., Stubenrauch, C. J., and Chatterjee, R.: Evaluation of upper tropospheric humidity forecasts from ECMWF using AIRS and CALIPSO data, Atmos. Chem. Phys., 9, 1779-1793, doi:10.5194/acp-9-1779-2009, 2009.

Lamquin, N., Stubenrauch, C. J., Gierens, K., Burkhardt, U., and Smit, H.: A global climatology of upper-tropospheric ice supersaturation occurrence inferred from the Atmospheric Infrared Sounder calibrated by MOZAIC, Atmos. Chem. Phys., 12, 381405, doi:10.5194/acp-12-381-2012, 2012. 
Lee, D., Pitari, G., Grewe, V., Gierens, K., Penner, J. E., Petzold, A., Prather, M. J., Schumann, U., Bais, A., Berntsen, T., Iachetti, D., Lim, L. L., and Sausen, R.: Transport impacts on atmosphere and climate: Aviation, Atmos. Environ., 44, 4678-4734, doi:10.1016/j.atmosenv.2009.06.005, 2010.

Lewellen, D. C.: Analytic solutions for evolving size distributions of spherical crystals or droplets undergoing diffusional growth in different regimes, J. Atmos. Sci., 69, 417-434, doi:10.1175/JASD-11-029.1, 2012.

Lewellen, D. C. and Lewellen, W. S.: Large-eddy simulations of the vortex-pair breakup in aircraft wakes, AIAA J., 34, 2337-2345, 1996.

Lewellen, D. C. and Lewellen, W. S.: The effects of aircraft wake dynamics on contrail development, J. Atmos. Sci., 58, 390-406, 2001

Lilly, D. K.: The representation of small-scale turbulence in numerical simulation experiments, in: IBM Sci. Comput. Symp. on Environm. Sci., 195-210, Thomas J. Watson Res. Center, Yorktown Heights, N.Y., IBM Form 320-1951, 1967.

Manney, G. L., Hegglin, M. I., Daffer, W. H., Santee, M. L., Ray, E. A., Pawson, S., Schwartz, M. J., Boone, C. D., Froidevaux, L., Livesey, N. J., Read, W. G., and Walker, K. A.: Jet characterization in the upper troposphere/lower stratosphere (UTLS): applications to climatology and transport studies, Atmos. Chem. Phys., 11, 6115-6137, doi:10.5194/acp-11-6115-2011, 2011.

Mannstein, H. and Schumann, U.: Aircraft induced contrail cirrus over Europe, Meteor. Z., 14, 549-554, 2005.

Mannstein, H., Meyer, R., and Wendling, P.: Operational detection of contrails from NOAA-AVHRR data, Int. J. Rem. Sens., 20, 1641-1660, 1999.

Mannstein, H., Spichtinger, P., and Gierens, K.: How to avoid contrail cirrus, Transp. Res., D 10, 421-426, 2005.

Mayer, B. and Kylling, A.: Technical note: The libRadtran software package for radiative transfer calculations - description and examples of use, Atmos. Chem. Phys., 5, 1855-1877, doi:10.5194/acp-5-1855-2005, 2005.

Meerkötter, R., Schumann, U., Doelling, D. R., Minnis, P., Nakajima, T., and Tsushima, Y.: Radiative forcing by contrails, Ann. Geophys., 17, 1080-1094, doi:10.1007/s00585-999-10807, 1999.

Meilinger, S. K., Kärcher, B., and Peter, Th.: Microphysics and heterogeneous chemistry in aircraft plumes - high sensitivity on local meteorology and atmospheric composition, Atmos. Chem. Phys., 5, 533-545, doi:10.5194/acp-5-533-2005, 2005.

Miake-Lye, R., Martinez-Sanchez, M., Brown, R., and Kolb, C. E.: Plume and wake dynamics, mixing and chemistry behind a high speed civil aircraft, J. Aircraft, 30, 467-479, 1993.

Minnis, P., Young, D. F., Garber, D. P., Nguyen, L., Smith Jr., W. L., and Palikonda, R.: Transformation of contrails into cirrus during SUCCESS, Geophys. Res. Lett., 25, 1157-1160, 1998.

Misaka, T., Holzäpfel, F., Hennemann, I., Gerz, T., Manhart, M., and Schwertfirm, F.: Vortex bursting and tracer transport of a counter-rotating vortex pair, Phys. Fluids, 24, 025104, doi:10.1063/1.3684990, 2012.

Mitchell, D. L. and Heymsfield, A. H.: Refinements in the treatment of ice particle terminal velocities, highlighting aggregates, J. Atmos. Sci., 62, 1637-1644, 2005.

Murphy, D. M. and Koop, T.: Review of the vapour pressures of ice and supercooled water for atmospheric applications, Q. J. Roy.
Meteor. Soc., 131, 1539-1565, doi:10.1256/qj.04.94, 2005.

Naiman, A. D., Lele, S. K., Wilkerson, J. T., and Jacobson, M. Z.: Parameterization of subgrid plume dilution for use in large-scale atmospheric simulations, Atmos. Chem. Phys., 10, 2551-2560, doi:10.5194/acp-10-2551-2010, 2010.

Naiman, A. D., Lele, S. K., and Jacobson, M. Z.: Large eddy simulations of contrail development: Sensitivity to initial and ambient conditions over first twenty minutes, J. Geophys. Res., 116, D21208, doi:10.1029/2011JD015806, 2011.

Nair, R. D., Scroggs, J. S., and Semazzi, F. H. M.: A forwardtrajectory global semi-Lagrangian transport scheme, J. Comput. Phys., 190, 275-294, doi:10.1016/S0021-9991(03)00274-2, 2003.

Nastrom, G. D. and Gage, K. S.: A climatology of atmospheric wavenumber spectra of wind and temperature observed by commerical aircraft, J. Atmos. Sci., 42, 950-960, 1985.

NIST: Guide for the use of the International System of Units (SI), Tech. rep., National Institute of Standards and Technology, Gaithersburg, MD 20899, NIST Special Publication 811, http://physics.nist.gov/cuu/pdf/sp811.pdf, 2008.

Paltridge, G. W. and Platt, C. M. R.: Radiative processes in meteorology and climatology, Elsevier, Amsterdam, 318 pp., 1976.

Paoli, R. and Garnier, F.: Interaction of exhaust jets and aircraft wake vortices: small-scale dynamics and potential microphysical-chemical transformations, Compt. Rend. Phys., 6, 525-547, 2005

Paoli, R., Vancassel, X., Garnier, F., and Mirabel, P.: Large-eddy simulation of a turbulent jet and a vortex sheet interaction: particle formation and evolution in the near field of an aircraft wake, Meteor. Z., 17, 131-144, doi:10.1127/0941-2948/2008/0278, 2008.

Paoli, R., Cariolle, D., and Sausen, R.: Review of effective emissions modeling and computation, Geosci. Model Dev., 4, 643 667, doi:10.5194/gmd-4-643-2011, 2011.

Paugam, R., Paoli, R., and Cariolle, D.: Influence of vortex dynamics and atmospheric turbulence on the early evolution of a contrail, Atmos. Chem. Phys., 10, 3933-3952, doi:10.5194/acp-103933-2010, 2010

Pavelin, E., Whiteway, J. A., Busen, R., and Hacker, J.: Airborne observations of turbulence, mixing, and gravity waves in the tropopause region, J. Geophys. Res., 107, 4084, doi:10.1029/2001JD000775, 2002.

Pisso, I., Real, E., Law, K. S., Legras, B., Bousserez, N., Attié, J. L., and Schlager, H.: Estimation of mixing in the troposphere from Lagrangian trace gas reconstructions during long-range pollution plume transport, J. Geophys. Res., 114, doi:10.1029/2008JD011289, 2009

Ploeger, F., Fueglistaler, S., Groö̈, J.-U., Günther, G., Konopka, P., Liu, Y. S., Müller, R., Ravegnani, F., Schiller, C., Ulanovski, A., and Riese, M.: Insight from ozone and water vapour on transport in the tropical tropopause layer (TTL), Atmos. Chem. Phys., 11, 407-419, doi:10.5194/acp-11-407-2011, 2011.

Ponater, M., Brinkop, S., Sausen, R., and Schumann, U.: Simulating the global atmospheric response to aircraft water vapour emissions and contrails: a first approach using a GCM, Ann. Geophys., 14, 941-960, doi:10.1007/s00585-996-0941-6, 1996.

Ponater, M., Marquart, S., and Sausen, R.: Contrails in a comprehensive global climate model: Parameterization and radiative forcing results, J. Geophys. Res., 107, 4164 
doi:10.1029/2001JD000429, 2002.

Pruppacher, H. R. and Klett, J. D.: Microphysics of Clouds and Precipitation, Kluwer Academic Publ., Dordrecht, 954 pp., 1997.

Rädel, G. and Shine, K. P.: Evaluation of the use of radiosonde humidity data to predict the occurrence of persistent contrails, Q. J. Roy. Meteor. Soc., 133, 1413-1423, doi:10.1002/qj.128, 2007, 2007.

Riley, J. J. and Lindborg, E.: Stratified turbulence: A possible interpretation of some geophysical turbulence measurements, J. Atmos. Sci., 65, 2416-2424, doi:10.1175/2007JAS2455.1, 2008.

Sarpkaya, T.: Trailing vortices in homogeneous and densitystratified media, J. Fluid Mech., 136, 85-109, 1983.

Sassen, K.: Contrail-cirrus and their potential for regional climate change, B. Am. Meteor. Soc., 78, 1885-1903, 1997.

Sausen, R., Gierens, K., Ponater, M., and Schumann, U.: A diagnostic study of the global distribution of contrails. Part I: Present day climate, Theor. Appl. Clim., 61, 127-141, 1998.

Schiller, C., Krämer, M., Afchine, A., Spelten, N., and Sitnikov, N.: Ice water content of Arctic, midlatitude, and tropical cirrus, J. Geophys. Res., 113, D24208, doi:10.1029/2008JD010342, 2008.

Schlager, H., Konopka, P., Schulte, P., Schumann, U., Ziereis, H., Arnold, F., Klemm, M., Hagen, D. E., Whitefield, P. D., and Ovarlez, J.: In situ observations of air traffic emission signatures in the North Atlantic flight corridor, J. Geophys. Res., 102, 10739-10750, 1997.

Schmidt, E.: Die Entstehung von Eisnebel aus den Auspuffgasen von Flugmotoren, in: Schriften der Deutschen Akademie der Luftfahrtforschung, Heft 44, 1-15, Verlag R. Oldenbourg, München, 1941.

Schoeberl, M. R., Douglass, A. R., Zhu, Z., and Pawson, S.: A comparison of the lower stratospheric age spectra derived from a general circulation model and two data assimilation systems, J. Geophys. Res., 108, 4113, doi:10.1029/2002JD002652, 2003.

Schröder, F., Kärcher, B., Duroure, C., Ström, J., Petzold, A., Gayet, J.-F., Strauss, B., Wendling, P., and Borrmann, S.: The transition of contrails into cirrus clouds, J. Atmos. Sci., 57, 464-480, 2000.

Schumann, U.: Subgrid length-scales for large-eddy simulation of stratified turbulence, Theor. Comput. Fluid Dyn., 2, 279-290, 1991.

Schumann, U.: On the effect of emissions from aircraft engines on the state of the atmosphere, Ann. Geophys., 12, 365-384, doi:10.1007/s00585-994-0365-0, 1994.

Schumann, U.: On conditions for contrail formation from aircraft exhausts, Meteor. Z., 5, 4-23, 1996.

Schumann, U.: Contrail cirrus, in: Cirrus, edited by Lynch, D. K., Sassen, K., O'C. Starr, D., and Stephens, G., 231-255, Oxford Univ. Press, Oxford, 2002.

Schumann, U.: A contrail cirrus prediction tool, in: Proceedings of the 2nd International Conference on Transport, Atmosphere and Climate (TAC-2), edited by: Sausen, R., van Velthoven, P. F. J., Brüning, C., and Blum, A., 69-74, Aachen, Germany, and Maastricht, The Netherlands, 22-25 June 2009, DLRForschungsbericht 2010-10, Cologne, Germany, ISSN 14348454, 2009.

Schumann, U. and Gerz:, T.: Turbulent mixing in stably stratified shear flows, J. Appl. Meteor., 34, 33-48, 1995.

Schumann, U. and Konopka, P.: A simple estimate of the concentration field in a flight corridor, in: Impact of Emissions from Aircraft and Spacecraft upon the Atmosphere. Proc. of an Intern.
Sci. Colloquium, Köln (Cologne), Germany, April 18-20, 1994, edited by: Schumann, U. and Wurzel, D., 354-359, DLR-Mitt. 94-06, 1994.

Schumann, U. and Wendling, P.: Determination of contrails from satellite data and observational results, in: Air Traffic and the Environment - Background, Tendencies and Potential Global Atmospheric Effects, edited by Schumann, U., 138-153, Springer, 1990.

Schumann, U., Konopka, P., Baumann, R., Busen, R., Gerz, T., Schlager, H., Schulte, P., and Volkert, H.: Estimate of diffusion parameters of aircraft exhaust plumes near the tropopause from nitric oxide and turbulence measurements, J. Geophys. Res., 100, 14147-14162, 1995.

Schumann, U., Ström, J., Busen, R., Baumann, R., Gierens, K., Krautstrunk, M., Schröder, F. P., and Stingl, J.: In situ observations of particles in jet aircraft exhausts and contrails for different sulfur-containing fuels, J. Geophys. Res., 101, 6853-6870, doi:10.1029/95JD03405, 1996.

Schumann, U., Schlager, H., Arnold, F., Baumann, R., Haschberger, P., and Klemm, O.: Dilution of aircraft exhaust plumes at cruise altitudes, Atmos. Env., 32, 3097-3103, 1998.

Schumann, U., Arnold, F., Busen, R., Curtius, J., Kärcher, B., Petzold, A., Schlager, H., Schröder, F., and Wohlfrom, K. H.: Influence of fuel sulfur on the composition of aircraft exhaust plumes: The experiments SULFUR 1-7, J. Geophys. Res., 107, 4247, doi:10.1029/2001JD000813, 2002.

Schumann, U., Mayer, B., Graf, K., Mannstein, H., and Meerkötter, R.: A parametric radiative forcing model for cirrus and contrail cirrus, in: ESA Atmospheric Science Conference, Barcelona, Spain, ESA SP-676, edited by: Agency, E. S., 1-6, Frascati, Italy, 2009.

Schumann, U., Mayer, B., Hamann, U., and Graf, K.: Radiative heating in contrail cirrus, Geophys. Res. Abstr., 12, EGU2010 1501-1, 2010.

Schumann, U., Graf, K., and Mannstein, H.: Potential to reduce the climate impact of aviation by flight level changes, in: 3rd AIAA Atmospheric and Space Environments Conference, AIAA paper 2011-3376, 1-22, 2011a.

Schumann, U., Mayer, B., Gierens, K., Unterstrasser, S., Jessberger, P., Petzold, A., Voigt, C., and Gayet, J.-F.: Effective radius of ice particles in cirrus and contrails, J. Atmos. Sci., 68, 300-321, doi:10.1175/2010JAS3562.1, 2011b.

Schumann, U., Mayer, B., Graf, K., and Mannstein, H.: A parametric radiative forcing model for contrail cirrus, J. Appl. Meteor. Climatol., 51, doi:10.1175/JAMC-D-11-0242.1, 2012.

Schwartz Dallara, E., Kroo, I. M., and Waitz, I.: Metric for comparing lifetime averaged climate impact of aircraft, AIAA J., 49, 1600-1613, 2011.

Scorer, R. S. and Davenport, L. J.: Contrails and aircraft downwash, J. Fluid Mech., 43, 451-464, 1970.

Sharman, R., Tebaldi, C., Wiener, G., and Wolff, J.: An integrated approach to mid- and upper-level turbulence forecasting, Weather Forecast., 21, 268-287, 2005.

Sölch, I. and Kärcher, B.: A large-eddy model for cirrus clouds with explicit aerosol and ice microphysics and Lagrangian ice particle tracking, Q. J. Roy. Meteor. Soc., 136B, 2074-2093, doi:10.1002/qj.689, 2010.

Sonntag, D.: Advancements in the field of hygrometry, Meteor. Z., 3, 51-66, 1994. 
Spichtinger, P., Gierens, K., and Dörnbrack, A.: Formation of ice supersaturation by mesoscale gravity waves, Atmos. Chem. Phys., 5, 1243-1255, doi:10.5194/acp-5-1243-2005, 2005a.

Spichtinger, P., Gierens, K., and Wernli, H.: A case studies of the formation and evolution of ice supersaturation in the vicinity of a warm conveyor belt's outflow region., Atmos. Chem. Phys., 5, 973-987, 2005b.

Spinhirne, J. D., Hart, W. D., and Duda, D. P.: Evolution of the morphology and microphysics of contrail cirrus from airborne remote sensing, Geophys. Res. Lett., 25, 1153-1156, 1998.

Stohl, A., Hittenberger, M., and Wotawa, G.: Validation of the Lagrangian particle dispersion model FLEXPART against largescale tracer experiment data, Atmos. Environ., 32, 4245-4264, 1998.

Stohl, A., Haimberger, L., Scheele, M. P., and Wernli, H.: An intercomparison of results from three trajectory models, Meteor. Appl., 8, 127-135, 2001.

Sun, Z.: Reply to comments by Greg M. McFarquhar, Q. J. Roy. Meteor. Soc., 127, 267-271, 2001.

Sun, Z. and Rikus, L.: Parametrization of effective sizes of cirruscloud particles and its verification against observations, Q. J. Roy. Meteor. Soc., 125, 3037-3055, 1999.

Sussmann, R. and Gierens, K.: Lidar and numerical studies on the different evolution of vortex pair and secondary wake in young contrails, J. Geophys. Res., 104, 2131-2142, 1999.

Sussmann, R. and Gierens, K.: Differences in early contrail evolution of two-engine versus four-engine aircraft: Lidar measurements and numerical simulations, J. Geophys. Res., 106, 48994911, 2001.

Tompkins, A., Gierens, K., and Rädel, G.: Ice supersaturation in the ECMWF Integrated Forecast System, Q. J. Roy. Meteor. Soc., 133, 53-63, doi:10.1002/qj.14, 2007.

Unterstrasser, S.: Numerische Simulationen von Kondensstreifen und deren Übergang in Zirren, Ph.D. thesis, University of Munich, Department of Physics, http://edoc.ub.uni-muenchen.de/ 9464, DLR-FB 2009-15, 2008.

Unterstrasser, S. and Gierens, K.: Numerical simulations of contrail-to-cirrus transition - Part 1: An extensive parametric study, Atmos. Chem. Phys., 10, 2017-2036, doi:10.5194/acp-102017-2010, 2010a.

Unterstrasser, S. and Gierens, K.: Numerical simulations of contrail-to-cirrus transition - Part 2: Impact of initial ice crystal number, radiation, stratification, secondary nucleation and layer depth, Atmos. Chem. Phys., 10, 2037-2051, doi:10.5194/acp-102037-2010, 2010b.
Unterstrasser, S. and Sölch, I.: Study of contrail microphysics in the vortex phase with a Lagrangian particle tracking model, Atmos. Chem. Phys., 10, 10003-10015, doi:10.5194/acp-1010003-2010, 2010.

Unterstrasser, S., Gierens, K., and Spichtinger, P.: The evolution of contrail microphysics in the vortex phase, Meteor. Z., 17, 145156, 2008.

van de Hulst, H. C.: Light Scattering by Small Particles, Wiley, New York, 470 pp., 1957.

Vazquez-Navarro, M. R.: Life cycle of contrails from a time series of geostationary satellite images, Ph.D. thesis, University of Munich, Department of Physics, http://edoc.ub.uni-muenchen. de/10913/, DLR-FB 2010-19, 2009.

Voigt, C., Schumann, U., Jurkat, T., Schäuble, D., Schlager, H., Petzold, A., Gayet, J.-F., Krämer, M., Schneider, J., Borrmann, S., Schmale, J., Jessberger, P., Hamburger, T., Lichtenstern, M., Scheibe, M., Gourbeyre, C., Meyer, J., Kübbeler, M., Frey, W., Kalesse, H., Butler, T., Lawrence, M. G., Holzäpfel, F., Arnold, F., Wendisch, M., Döpelheuer, A., Gottschaldt, K., Baumann, R., Zöger, M., Sölch, I., Rautenhaus, M., and Dörnbrack, A.: In-situ observations of young contrails - overview and selected results from the CONCERT campaign, Atmos. Chem. Phys., 10, 90399056, doi:10.5194/acp-10-9039-2010, 2010.

Voigt, C., Schumann, U., Jessberger, P., Jurkat, T., Petzold, A., Gayet, J.-F., Krämer, M., Thornberry, T., and Fahey, D. W.: Extinction and optical depth of contrails, Geophys. Res. Lett., 38, L11806, doi:10.1029/2011GL047189, 2011.

Wernli, H. and Davies, H. C.: A Lagrangian-based analysis of extratropical cyclones. I: The method and some applications, Q. J. Roy. Meteor. Soc., 123, 467-489, 1997.

Wilkerson, J. T., Jacobson, M. Z., Malwitz, A., Balasubramanian, S., Wayson, R., Fleming, G., Naiman, A. D., and Lele, S. K.: Analysis of emission data from global commercial aviation: 2004 and 2006, Atmos. Chem. Phys., 10, 6391-6408, doi:10.5194/acp-10-6391-2010, 2010.

Wong, H.-W. and Miake-Lye, R. C.: Parametric studies of contrail ice particle formation in jet regime using microphysical parcel modeling, Atmos. Chem. Phys., 10, 3261-3272, doi:10.5194/acp-10-3261-2010, 2010. 